THE COST OF RISK AND OPTION HEDGING IN INCOMPLETE MARKETS 


\section{Graduation committee:}

prof.dr.ir. A.J. Mouthaan(chairman), University of Twente prof.dr. A. Bagchi(promotor), University of Twente dr.ir. M.H. Vellekoop(assistant promotor), University of Twente prof.dr. R.J. Boucherie, University of Twente prof.dr. F. Jamshidian, University of Twente dr.ir. N. van den Hijligenberg, Sfiss Financial Technologies dr. J.W. Nieuwenhuis, University of Groningen prof.dr. A.A.J. Pelsser, University of Amsterdam 


\title{
THE COST OF RISK AND OPTION HEDGING IN INCOMPLETE MARKETS
}

\author{
DISSERTATION
}

to obtain

the degree of doctor at the University of Twente, on the autority of the rector magnificus, prof.dr. W.H.M. Zijm,

on account of the decision of the graduation committee, to be publicly defended on Thursday, 10 January 2008 at 15:00

by

Vera Minina

born on 23 January 1979

in Moscow, Russia 
This dissertation has been approved by:

prof.dr. A.Bagchi (promotor)

dr.ir. M.H.Vellekoop (assistant promotor) 


\section{Acknowledgments}

First of all I would like to express my gratitude to my promotor Prof. Arun Bagchi who offered me this $\mathrm{PhD}$ position. I thank him for his valuable scientific advice as well as for being very flexible and accommodating in all the practical matters. I would also like to thank my daily supervisor and assistant promotor Michel Vellekoop for his guidance, support and inspiring ideas through the whole period of my $\mathrm{PhD}$ project.

I thank the members of my promotion committee: Prof. F. Jamshidian, Prof. A. Pelsser, Dr. N. van den Hijligenberg, Prof. R. Boucherie and Dr. J. Nieuwenhuis for their time and effort in reviewing my thesis and their valuable comments that very much improved the script.

I thank Bastiaan de Geeter and Julien Gosme from Saen Options for their regular feedback and advise. I also thank Sfiss Financial Technologies and all people working there for giving me an opportunity to work in their office in Amsterdam. I also thank my colleagues from Systems, Signals and Control Group and FELab for making my working in the University enjoyable.

I am grateful to my husband Alex Zilber not only for his love and moral support but also for some very useful research ideas. I would also like to thank my friends Maria Kholopova, Zaher Daher, Aafje Ouwehand, Maarten Hummel, Ivan Asinovsky and my sisters Sonia Minina and Olia Minina for being there for me and making my life pleasant and interesting. Finally I would like to thank my parents for their love and unconditional support. 


\section{Contents}

$\begin{array}{ll}\text { Acknowledgments } & 5\end{array}$

$\begin{array}{lr}\text { List of Notations } & 9\end{array}$

1 Introduction 11

2 Complete Markets and Hedging Portfolios $\quad 15$

2.1 Market Models and Contingent Claims . . . . . . . . . . . 15

2.2 Self-Financing Portfolios and Arbitrage . . . . . . . . . . . 17

2.3 Arbitrage Pricing and Complete Markets . . . . . . . . . . . . 18

2.4 Arbitrage Pricing and Hedging in the

Generalized Black-Scholes Model . . . . . . . . . . . . . 19

2.5 Martingale Measures and Risk-Neutral

Valuation .................... . . 21

2.6 Risk-Neutral Valuation in the Black-Scholes Model . . . . . . 23

3 Incomplete Models $\quad 29$

3.1 Volatility Smiles . . . . . . . . . . . . . . . . . 30

3.2 Quadratic Hedging . . . . . . . . . . . . . . . . . . 32

3.3 Utility Functions and Indifference Pricing . . . . . . . . . 36

4 Mean-Variance Hedging for Black-Scholes Dynamics 41

4.1 Formulation of Hedging Problem . . . . . . . . . . . . . . 41

4.2 Dynamic Programming Problem . . . . . . . . . . . . . . 43

4.3 Properties of the Cost Function and the Recursive Algorithm . 45

4.4 The Optimal Initial Capital . . . . . . . . . . . . . . . . . 49

4.5 Continuous Dynamics . . . . . . . . . . . . . . 52

5 Model with Cost of Risk $\quad 61$

5.1 The Model . . . . . . . . . . . . . . . . . . . . 63

5.1.1 The Optimization Problem Setup . . . . . . . . . 65

5.1 .2 The Risk Measure . . . . . . . . . . . . . . . . . 68 
5.2 The Dynamic Programming Algorithm . . . . . . . . . . . 71

5.3 Analytical Solutions for the Indifference Prices . . . . . . . . . 73

5.4 Absence of Arbitrage . . . . . . . . . . . . . . . . . 79

6 Numerical Results $\quad 85$

6.1 Indifference Prices . . . . . . . . . . . . . . . . . . . . . . . 85

6.2 Calibration to the Market Data . . . . . . . . . . . . . 87

6.2.1 Parameters' Interpretation . . . . . . . . . . . . . 87

6.2.2 Calibration Algorithm . . . . . . . . . . . . . . . 90

6.2.3 Calibration Results . . . . . . . . . . . . . . 93

7 Conclusions and Discussion 103 


\section{List of Notations}

$\begin{array}{ll}\Omega & \text { event space } \\ \mathcal{F} & \sigma \text {-algebra of events } \\ \left\{\mathcal{F}_{t}\right\}_{t=0}^{T} & \text { filtration } \\ m \mathcal{F}_{t} & \text { the set of } \mathcal{F}_{t} \text {-measurable random variables } \\ \mathbb{P}, \mathbb{Q} & \text { probability measures } \\ \frac{d \mathbb{Q}}{d \mathbb{P}} & \text { Radon-Nikodym derivative of measure } \mathbb{Q} \text { with respect to } \\ \mathbf{E}^{\mathbb{P}}[X] & \text { measure } \mathbb{P} \\ \mathbf{E}_{t}[X] & \text { expectation of random variable } X \text { under measure } \mathbb{P} \\ \mathcal{T} & \text { expectation of random variable } X \text { conditional on } \mathcal{F}_{t} \\ X & \text { time set } \\ X^{i} & \text { stochastic process } \\ \theta & \text { the } i \text { th coordinate of vector process } X \\ V & \text { portfolio process } \\ V^{\theta} & \text { value of a portfolio } \\ W & \text { value of portfolio process } \theta \\ S & \text { Brownian motion } \\ B & \text { risky asset price process } \\ r & \text { regular bank account price process } \\ Z & \text { risk-free rate } \\ \tilde{r} & \text { reserve bank account price process } \\ \prod_{t} & \text { reserve bank account rate } \\ \Phi & \text { pricing rule at time } t \\ \Phi^{i} & \text { contingent claim payoff } \\ \Phi_{k} & \text { payoff of the } i \text { th option in the portfolio } \\ U & \text { payoff at time } t_{k} \text { of a portfolio of options } \\ t_{k} & \text { utility function } \\ \Delta t_{k}=t_{k+1}-t_{k} & k \text { th time point of a discrete model } \\ X_{k}=X\left(t_{k}\right) & k \text { th time step } \\ \Delta X_{k}=X_{k+1}-X_{k} & \text { stochastic process in a discrete model } \\ \vec{e}=(1, \ldots, 1)^{T} & k \text { th increment of process } X \\ & \text { vector consisting of ones } \\ & \\ & \end{array}$


$R_{k}=S_{k+1} / S_{k} \quad$ the $k$ th return on the risky asset $S$

$\{\Phi\}_{\{\Psi\}, k}^{s} \quad$ indifference selling price at time $t_{k}$ of $\Phi$ in presence of $\Psi$ $\{\Phi\}_{\{\Psi\}, k}^{b} \quad$ indifference buying price at time $t_{k}$ of $\Phi$ in presence of $\Psi$ $\{\Phi\}_{\{\Psi\}, k}^{m} \quad$ indifference mid price at time $t_{k}$ of $\Phi$ in presence of $\Psi$ $\operatorname{Bin}_{k}^{i, \Phi_{i}} \quad$ binomial price at time $t_{k}$ of contingent claim $\Phi_{i}$ payable at time $t_{i} \geq t_{k}$

$\hat{\Delta}_{k}^{\Phi}$ binomial Delta of portfolio $\Phi$ at time $t_{k}$

$\mathcal{V}_{k}$ Black-Scholes Vega of portfolio $\Phi$ at time $t_{k}$ vanna $_{k}$ Black-Scholes Vanna of portfolio $\Phi$ at time $t_{k}$ $\operatorname{volga}_{k}$ $\rho_{k}$ Black-Scholes Volga of portfolio $\Phi$ at time $t_{k}$ risk function at time $t_{k}$ 


\section{Chapter 1}

\section{Introduction}

Option pricing became an important part of mathematical research in 1973 after the publication of the famous Black-Scholes formula [9], which gives an analytical solution for the price of a European option under certain conditions. When these conditions hold one is able to construct an option from trading other assets following certain rules. The idea is to set up a portfolio that is divided between a risk-free bank account and a position in the underlying asset in order to replicate the payoff of the option. To achieve this the proportions invested in the underlying and the bank account are continuously rebalanced according to differential equations derived from the model chosen to describe the market. This process is called hedging. If the assumptions of Black and Scholes were correct, there would be no risk in selling options as they could be replicated perfectly. However the BlackScholes assumptions do not hold in the real world and a perfect hedge is not possible. This means that there is always some degree of risk that the final value of the hedging portfolio and the option payoff do not match.

The fact that in real life a perfect hedge is not possible is acknowledged by both practitioners and academics. It is understood that models taking this aspect into account should be built. There are two main approaches described in the literature. The first way is to make the risk as small as the model allows. Here one may think of minimizing the variance of the difference between the hedging portfolio and the option payoff. While this approach minimizes risk, it does not take care of the difference between profit and loss, which may happen highly negative. Mean-variance hedging - the approach that minimizes the expected squared difference between the hedging portfolio and the option payoff (see for example [45]) takes care of this problem but has a different drawback - it punishes the trader for an extra profit he or she might gain. Therefore a second way to deal with this risk has been developed - maximization of the profit while making sure the risk does not become too 
high. The utility function approach allows one to do that. The maximization of the expected utility gives one a strategy that maximizes the profit while taking account of risk. Nevertheless despite thorough research made in this field and the fact that it is well-developed from a theoretical point of view, the utility approach is rarely used in practice mostly due to the difficulty of defining the utility function of a trader.

The goal of this thesis is to explore the possibilities of constructing a model that would reflect real life hedging better. In practice traders do not hedge continuously because transaction costs make that infinitely expensive and because of the traders' physical limitations. Moreover traders do not always aim at hedging all the risk. Sometimes they maintain not fully hedged, risky positions in an attempt to maximize their profits. These two phenomena, discrete hedging and profit maximization, are two main points that we address in our study.

First we study an agent who, although he or she is not allowed to trade continuously, still tries to hedge the contingent claim as closely as possible by minimizing the expected squared difference, or the distance, between the option payoff and the value of the hedging portfolio. In this part of our thesis we follow the line of Schweizer's mean-variance hedging. Under the assumption of independent stock returns we are able to find an explicit recursion formulae for the optimization problem. The optimal initial capital is the expectation of the option payoff under a certain measure called the variance-optimal measure. We prove that if the distance between hedging dates goes to zero then the variance-optimal measure converges to the riskneutral measure of the limiting Black-Scholes model and the optimal initial capital converges to the Black-Scholes price.

Next, we construct a model with a cost of risk where a trader maximizes his or her profit from hedging a portfolio of European options without using a utility function. The limitation on the trader is imposed by a risk function that depends on the the market state and the portfolio. According to the value of this function, the trader is required to set aside some money as a reserve. This reserve is modeled by an additional bank account paying an interest rate lower than the risk-free one. The higher the risk of the trader the more he or she has to borrow from the regular bank account in favor of the reserve and the more he or she loses because of the lower interest rate. The trader maximizes the expectation of his or her portfolio value at the final point.

The prices of our model are defined in the indifference way. The indifference buying and selling prices of a portfolio of options $\Phi$ in the presence of another portfolio of options $\Psi$ are defined as the amount of money that makes an optimally behaving trader indifferent between buying or selling 
portfolio $\Phi$ and not buying or selling it, while holding portfolio $\Psi$. These prices depend on the trader's current portfolio and allow him or her to give competitive quotes. They also depend on the direction of the quote (buy/sell) and therefore the model produces bid-ask spreads. For a special form of the risk function dependent on the portfolio Greeks, or sensitivities of the portfolio value to the market parameters, we are able to solve the optimization problem explicitly and find the analytic formulae for the indifference prices. The mid indifference prices, or the average of the buying and the selling prices, can be calibrated to the market.

This thesis is structured as follows. Chapter 2 introduces the basic definitions of contingent claims, arbitrage pricing and martingale measures and presents a brief overview of pricing and hedging in complete markets. Chapter 3 introduces the concept of implied volatility smiles and presents two approaches to the optimal hedging in incomplete markets: the mean-variance hedging and the utility approach. Chapter 4 discusses mean-variance hedging and presents the convergence result. In chapter 5 we introduce our model with cost of risk and the indifference prices and discuss the choice of the risk function. Chapter 6 contains the numerical results for the model with cost of risk. It presents some examples of the indifference prices and the results of the calibration of the model to the market. We conclude the thesis with a discussion and some directions for further research in chapter 7 . 


\section{Chapter 2}

\section{Complete Markets and Hedging Portfolios}

In chapter 2 we introduce the basic notions of a market model, a contingent claim, a hedging strategy and an arbitrage opportunity. We define a complete market model and show how the no-arbitrage assumption leads to the prices of contingent claims. We define the notion of an equivalent martingale measure and show that in a complete model the prices of contingent claims are equal to their expectations under a certain equivalent martingale measure.

\subsection{Market Models and Contingent Claims}

We consider a financial market consisting of $m+1$ assets $-m$ risky stocks $S^{1}, \ldots, S^{m}$ and a riskless bank account $B$. In this section we assume that our market satisfies the following basic conditions. First, it is allowed to buy, sell or hold any amount of an asset - also fractional, irrational and negative amounts. Holding a negative number of a certain asset corresponds to a short position in this asset. Next, unless stated otherwise the market is frictionless, meaning that the trader does not have to pay any extra money for a transaction and there are no bid-ask spreads (the buying and selling prices are the same). And third, the market is absolutely liquid - one may always buy or sell any amount of asset at its current price, and the buying and the selling have no effect on existing prices. In this chapter we are focusing on continuous-time models, which we mathematically define as follows.

Definition 2.1.1 We call a market model a probability space $(\Omega, \mathcal{F}, P)$ with a continuous filtration $\left\{\mathcal{F}_{t}\right\}_{0 \leq t \leq T}$, where $0 \leq T<+\infty$ is a finite 
time horizon. The zero-dividend stock prices $S^{k}(t)$ are adapted nonnegative stochastic processes $S^{k}:[0, T] \times \Omega \rightarrow \mathbb{R}^{+}$, which are assumed to be semimartingales, and the bank account process $B(t)$ is a positive deterministic function of time $B:[0, T] \rightarrow \mathbb{R}^{+}$.

For some of the examples of market models we refer the reader to the Black-Scholes model (see [9] and [33]), local volatility model (see [15] and [17]) and stochastic volatility model (see [24]).

The main problems in financial mathematics are the pricing and the hedging of financial contracts called options. The simplest examples of options are European Puts and Calls. These are contracts that give their holder a right to buy (if it is a Call) or to sell (if it is a Put) a predetermined asset (the underlying) at a predetermined date (the maturity) at a predetermined price (the strike). These options are also called plain vanilla options. The more complicated contracts are called exotic options. American Puts and Calls are the rights to buy or to sell a unit of stock at a certain price but at any time before or on the maturity date. Asian options are options with a payoff that depends on some average value of the underlying asset over a certain time period. A knock-in (a knock-out) barrier option is a contract, where the holder gets (loses) the right to sell or to buy the underlying asset for the strike price if the underlying asset has hit a certain level (the barrier) before the maturity date. The value of a European Call on asset $S^{i}$ at the time of its maturity $T$ is

$$
\max \left(S^{i}(T)-K, 0\right)
$$

where $K$ is the strike. This value is also called the payoff of the option. If $K=S^{i}(t)$, then at time $t$ the option is called at the money. If $K>S^{i}(t)$, then at time $t$ the option is called in the money if it is a Put or out of the money if it is a Call. And vice versa a Call with $K<S^{i}(t)$ is called in the money and a Put with $K<S^{i}(t)$ is called out of the money.

Finding the option value or a "fair" amount of money which has to be exchanged between the buyer and the seller of the option at some time before maturity is called option pricing. By buying or selling an option the trader accepts certain risks. Trading in the basic market assets in order to reduce these risks is called hedging. Throughout this thesis we are mainly interested in the pricing and the hedging of vanilla options and other European instruments. Therefore we use the following definition of a contingent claim.

Definition 2.1.2 We call at-contingent claim any integrable $\mathcal{F}_{t}$-measurable random variable. An $\mathcal{F}_{t}$-measurable random variable $X$ of the form 
$X=\Phi\left(S^{1}(t), \ldots, S^{m}(t)\right)$, where $\Phi: \mathbb{R}^{m} \rightarrow \mathbb{R}$ is a given (measurable) function, is called a simple contingent claim. Unless mentioned otherwise, under a contingent claim we understand a T-contingent claim.

For example the payoff of a European Call on the $i$ th asset is a simple contingent claim.

\subsection{Self-Financing Portfolios and Arbitrage}

In this section we define a self-financing trader's portfolio and the notion of arbitrage. All these definitions are standard definitions of mathematical finance and can be found for example in [8].

An agent trading in the market at each point in time has a position in various traded assets. This position is called the trader's portfolio. We model the portfolio by means of an $m+1$-dimensional real valued stochastic vector process $\theta(t)=\left(\psi(t), \varphi^{1}(t), \ldots, \varphi^{m}(t)\right)$, where the predictable $\varphi^{i}(t)$ is the number of the $i$ th assets in the portfolio and the adapted $\psi(t)$ is the amount of cash in the bank account at time $t$. The value $V^{\theta}$ of the portfolio at time $t$ is

$$
V^{\theta}(t)=\sum_{i=1}^{m} \varphi^{i}(t) S^{i}(t)+\psi(t)
$$

We restrict the set of portfolio processes to avoid some theoretically possible but practically very unreasonable situations (e.g. winning in a casino by always doubling the bets provided an unlimited credit is available). We consider only strategies with gains that are bounded from below.

Definition 2.2.1 A portfolio process $\theta(t)=\left(\psi(t), \varphi^{1}(t), \ldots, \varphi^{m}(t)\right)$ is called admissible if there exists $\alpha \geq 0$ (which may depend on $\varphi$ ) such that

$$
\sum_{i=1}^{m} \int_{0}^{t} \varphi^{i}(u) d S^{i}(u) \geq-\alpha \quad \text { for all } t \in[0, T]
$$

There is a special very important class of admissible portfolios which are called self-financing. We call an admissible portfolio self-financing if there is no infusion or withdrawal of money from the outside. The changes in value of such portfolios are only due to the changes of the assets prices. 
Definition 2.2.2 We call an admissible portfolio process self-financing if the dynamics of its value process for all $t \in[0, T]$ satisfy

$$
d V^{\theta}(t)=\sum_{i=1}^{m} \varphi^{i}(t) d S^{i}(t)+\psi(t) \frac{d B(t)}{B(t)} .
$$

One of the key concepts of mathematical finance is the concept of arbitrage. Loosely speaking, arbitrage is the possibility of getting a sure positive profit without any initial investment. This might happen, for example, if one trader quotes a lower price for an asset than another. Then buying the asset from the first trader and immediately selling it to the second one would produce a sure positive profit.

Definition 2.2.3 We call a self-financing portfolio process $\theta(t)$ an arbitrage opportunity in the financial market if the value $V^{\theta}(t)$ of this portfolio satisfies

$$
\begin{aligned}
V^{\theta}(0) & =0 \\
P\left(V^{\theta}(T)>0\right) & >0 \\
P\left(V^{\theta}(T)<0\right) & =0 .
\end{aligned}
$$

In practice these opportunities are very rare and if they happen they are of very short duration. Indeed, the goal of each investor is to make his or her profit as large as possible under a certain risk constraint. An arbitrage opportunity is a riskless profit. Therefore if there is an arbitrage somewhere in the market all investors who see it immediately begin buying or selling the mispriced asset. The big orders very quickly drag the price back to its equilibrium value. Therefore most market models work under the assumption that arbitrage is not possible. Throughout this thesis we also assume:

Assumption 1 (The No Arbitrage Assumption) Price processes in our market models do not allow arbitrage opportunities.

\subsection{Arbitrage Pricing and Complete Markets}

Finding the "fair" value of a contingent claim is one of the most important issues for the options traders. The concept of the no-arbitrage price or the price that does not create arbitrage opportunities is a way of formalizing the "fairness". First we give the definition of a general valuation procedure. 
Definition 2.3.1 We call a pricing rule a mapping $\Pi_{t}: m \mathcal{F}_{T} \rightarrow \mathbb{R}$, which for any contingent claim $H$ assigns a value $\Pi_{t}(H) \in \mathbb{R}$ at all points of time $t \in[0, T]$ such that $\Pi$. $(H)$ is an adapted process with $\Pi_{t}(H)=0$ if and only if $H=0$ almost surely. The pricing rule is called consistent with the model if

$$
\Pi_{t}\left(V^{\theta}(T)\right)=V^{\theta}(t)
$$

for all self-financing strategies $\theta$.

Let us fix a contingent claim $X$ and imagine that we are able to construct a portfolio $\theta^{X}=\left(\psi^{X}, \varphi^{X}\right)$ such that its terminal value is equal to the contingent claim payoff:

$$
V^{\theta^{X}}(T)=X
$$

then we say that contingent claim $X$ is attainable. Note that in an arbitragefree market the price of an attainable contingent claim $X$ is unique and equal to the value of its replicating portfolio $V^{\theta^{X}}$ :

$$
\Pi_{t}(X)=V^{\theta^{X}}(t)
$$

This can be easily seen as follows. Suppose, $\Pi_{t}(X)>V^{\theta^{X}}(t)$. Then selling a unit of option, buying the portfolio and putting the rest of cash on the bank account at time $t$ has value zero at time $t$ and a sure positive value at maturity, which is an arbitrage strategy. Since we do not allow that in our market, $\Pi_{t}(X) \leq V^{\theta^{X}}(t)$. A similar construction shows that the price cannot be strictly greater than the value of the portfolio and hence (2.1) is true. Therefore if we know how to replicate a contingent claim, we immediately know its no-arbitrage price. This procedure is called arbitrage pricing.

Definition 2.3.2 A market model is said to be complete, if all contingent claims bounded from below are attainable.

If the model is complete then for the pricing of a general contingent claim $X$ it is enough to find its replicating portfolio $\theta^{X}$ and set the price of $X$ to be equal the value of $\theta^{X}$.

\subsection{Arbitrage Pricing and Hedging in the Generalized Black-Scholes Model}

Let us show how arbitrage pricing works for a special case of a market model — the generalized Black-Scholes model (see [9]). We specify the model 
by defining two asset price processes $B(t)$ and $S(t)$ on our earlier introduced filtered probability space $\left(\Omega, \mathcal{F},\left\{\mathcal{F}_{t}\right\}_{0 \leq t \leq T}, \mathbb{P}\right)$. $B(t)$ and $S(t)$ have the following dynamics

$$
\begin{aligned}
d B(t) & =r(t) B(t) d t \\
B(0) & =1 \\
d S(t) & =\mu(t, S(t)) S(t) d t+\sigma(t, S(t)) S(t) d W(t), \\
S(0) & =S_{0}
\end{aligned}
$$

where $r:[0, T] \rightarrow \mathbb{R}^{+}, \mu:[0, T] \times \mathbb{R} \rightarrow \mathbb{R}, \sigma:[0, T] \times \mathbb{R} \rightarrow \mathbb{R}$ are given functions and functions $\hat{\mu}(t, s)=s \mu(t, s)$ and $\hat{\sigma}(t, s)=s \sigma(t, s)$ satisfy

$$
\begin{aligned}
|\hat{\mu}(t, x)|+|\hat{\sigma}(t, x)| & \leq C(1+|x|) \\
|\hat{\mu}(t, x)-\hat{\mu}(t, y)|+|\hat{\sigma}(t, x)-\hat{\sigma}(t, y)| & \leq D|x-y|
\end{aligned}
$$

for all $x, y \in \mathbb{R}, t \in[0, T]$ and some constants $C, D \in \mathbb{R}$. $W$ is a standard $\mathcal{F}_{t}$-Brownian motion. We consider a simple contingent claim $X=\Phi(S(T))$, where $\Phi: \mathbb{R} \rightarrow \mathbb{R}$ is a measurable function.

Suppose now that $F:[0, T] \times \mathbb{R} \rightarrow \mathbb{R}$ is continuous and of class $C^{1,2}([0, T] \times$ $\mathbb{R})$ and solves the Cauchy problem (2.6).

$$
\begin{aligned}
\frac{\partial F}{\partial t}+r S \frac{\partial F}{\partial s}+\frac{1}{2} \sigma^{2} s^{2} \frac{\partial^{2} F}{\partial s^{2}}-r F & =0 \\
F(T, s) & =\Phi(s),
\end{aligned}
$$

where $\Phi$ is continuous in $\mathbb{R}$ and

$$
|\Phi(x)| \leq A\left(1+|x|^{\alpha}\right)
$$

for some $A, \alpha>0$. Equation (2.6) is called the Black-Scholes equation. Note that under the above model assumptions such $F(t, s)$ exists (see [20]). Let us define a portfolio $\theta(t)=(\varphi(t), \psi(t))$ by

$$
\begin{aligned}
\varphi(t) & =\frac{\partial F}{\partial s}(t, S(t)) \\
\psi(t) & =F(t, S(t))-S(t) \frac{\partial F}{\partial s}(t, S(t))
\end{aligned}
$$

for all $t \in[0, T]$. We claim that

(1) the portfolio $\theta$ is the hedging portfolio for contingent claim $X$ and

(2) its price process $V^{\theta}$ is given by

$$
V^{\theta}(t)=F(t, S(t))
$$


Note that (2) obviously follows from the definition of the portfolio value:

$$
V^{\theta}(t)=\varphi(t) S(t)+\psi(t)=F(t, S(t)) .
$$

Now, to see (1) we have to prove that $\theta(t)$ is self-financing and that the final value of the portfolio coincides with the contingent claim payoff. Since $F(t, S(t))$ is the solution to (2.6) and from (2.7) the final value of the portfolio is equal to $F(T, S(T))=\Phi(S(T))$, the payoff of contingent claim $X$. Let us finally show that $\theta(t)$ is self-financing. Consider its value process $V^{\theta}(t)$. By Itô's rule we may write:

$$
\begin{aligned}
d V^{\theta}(t) & =d F(t, S(t)) \\
& =\left(\frac{\partial F}{\partial t}+\mu S \frac{\partial F}{\partial s}+\frac{1}{2} \sigma^{2} S^{2} \frac{\partial^{2} F}{\partial s^{2}}\right) d t+\sigma S \frac{\partial F}{\partial s} d W(t)
\end{aligned}
$$

Now remembering again that $F(t, S(t))$ is the solution to (2.6) we have

$$
\begin{aligned}
d V^{\theta}(t) & =r F(t, S(t)) d t+(\mu-r) S \frac{\partial F}{\partial s} d t+\sigma S \frac{\partial F}{\partial s} d W(t) \\
& =\left(F-S \frac{\partial F}{\partial s}\right) r d t+\frac{\partial F}{\partial s} d S(t)
\end{aligned}
$$

which is exactly

$$
d V^{\theta}(t)=\varphi(t) d S(t)+\psi(t) \frac{d B(t)}{B(t)}
$$

and therefore $\theta$ is self-financing.

By the above reasoning we have shown that any simple contingent claim in the generalized Black-Scholes model is attainable. Therefore its price is equal to the value of its replicating portfolio, it is unique and does not depend on individual preferences of the trader. Note that it also does not depend on the drift $\mu$ of the underlying asset. Both of these facts - the uniqueness and the independence of the drift - are not accidental and are corollaries of a more general theory we are going to present in the next sections.

\subsection{Martingale Measures and Risk-Neutral Valuation}

The notion of arbitrage pricing led us to the option price as the solution of a certain backward partial differential equation. There is another approach 
to arbitrage pricing where the contingent claim prices are calculated as conditional expectations under a special probability measure called the equivalent martingale measure. If the equivalent martingale measure is unique it is called the risk-neutral measure and this pricing method (producing unique no-arbitrage prices) is called risk-neutral valuation.

Imagine we have to price a contingent claim, and its price process is known to be a martingale. Then the only thing we have to do to find prices is to take a conditional expectation of its final payoff. Unfortunately the martingale property does not hold for the real-life prices. Nevertheless we may still use this technique if we are able to find a measure $\mathbb{Q}$ (equivalent to $\mathbb{P})$ such that the discounted price processes in the new model $(\Omega, \mathcal{F}, \mathbb{Q})$ become martingales.

Definition 2.5.1 A measure $\mathbb{Q}$ on a measurable space $(\Omega, \mathcal{F})$ is said to be absolutely continuous with respect to a measure $\mathbb{P}$ on $\mathcal{F}(\mathbb{Q}<<\mathbb{P})$ if for all $A \in \mathcal{F}$

$$
\mathbb{P}(A)=0 \Rightarrow \mathbb{Q}(A)=0 .
$$

If we have both $\mathbb{Q}<<\mathbb{P}$ and $\mathbb{P}<<\mathbb{Q}$, then $\mathbb{Q}$ and $\mathbb{P}$ are said to be equivalent $(\mathbb{Q} \sim \mathbb{P})$.

Definition 2.5.2 A measure $\mathbb{Q}$ on $(\Omega, \mathcal{F})$ is said to be an equivalent martingale measure if

- $\mathbb{Q}$ is equivalent to $\mathbb{P}$.

- The discounted spot price processes $S^{i}(t) / B(t), i=1, \ldots, m$ are $\mathcal{F}_{t^{-}}$ martingales under $\mathbb{Q}$.

The following proposition shows that defining an equivalent martingale measure $\mathbb{Q}$ is equivalent to defining a pricing rule $\Pi_{t}$ consistent with the model.

Proposition 2.5.1 There is a one-to-one correspondence between linear pricing rules $\Pi_{t}$ consistent with the model and equivalent martingale measures $\mathbb{Q}$ via

(i) $\Pi_{t}(X)=\boldsymbol{E}^{\mathbb{Q}}\left(\frac{B(t)}{B(T)} X \mid \mathcal{F}_{t}\right)$,

(ii) $\mathbb{Q}(A)=\Pi_{0}\left(\frac{B(T)}{B(0)} \boldsymbol{1}_{A}\right)$

for any contingent claim $X$ and $A \in \mathcal{F}$. 
This proposition is proved in Harrison and Pliska [23] for a model with discrete time set and finite state space. The more general version of the proposition is a corollary of the fundamental theorem presented in Delbaen and Schachermayer [14].

Note that the pricing rule $\Pi_{t}$ defined in proposition 2.5.1 produces arbitragefree prices. Let there exist an arbitrage opportunity $\theta$ with $V^{\theta}(0)=0$, $\mathbb{P}\left(V^{\theta}(T)<0\right)=0$ and $\mathbb{P}\left(V^{\theta}(T)>0\right)>0$. Then (since $\left.\mathbb{Q} \sim \mathbb{P}\right)$ we have that

$$
\begin{aligned}
& \mathbb{Q}\left(V^{\theta}(T)<0\right)=0 \\
& \mathbb{Q}\left(V^{\theta}(T)>0\right)>0 .
\end{aligned}
$$

Then

$$
\Pi_{0}\left(V^{\theta}(T)\right)=\mathbf{E}^{\mathbb{Q}}\left(\frac{B(0)}{B(T)} X\right)>0
$$

and since by proposition 2.5.1 $\Pi_{t}$ is consistent with the model

$$
V^{\theta}(0)=\Pi_{0}\left(V^{\theta}(T)\right)>0 .
$$

Hence the arbitrage opportunities do not exist and $\Pi_{t}$ does not produce arbitrage. So if a martingale measure exists, then taking conditional expectations under it provides us with arbitrage-free prices. If this measure is unique, then it is called the risk-neutral measure and this pricing procedure is referred to as the risk-neutral valuation. We will discuss an example of this method in the next section.

\subsection{Risk-Neutral Valuation in the Black-Scholes Model}

Let us show how risk-neutral valuation works on the example of a European Call in the generalized Black-Scholes model (2.2)-(2.3). To find the equivalent martingale measure we use Girsanov's theorem.

Theorem 2.6.1 (Girsanov, one dimension) Let $W(t)$ be a Brownian motion on a probability space $(\Omega, \mathcal{F}, \mathbb{P})$ with respect to the filtration $\left\{\mathcal{F}_{t}\right\}_{t=0}^{T}$. Let $\Theta(t)$ be an adapted process. Define

$$
\begin{aligned}
Z(t) & =\exp \left\{-\int_{0}^{t} \Theta(s) d W(s)-\frac{1}{2} \int_{0}^{t} \Theta^{2}(s) d s\right\}, \\
\tilde{W}(t) & =W(t)+\int_{0}^{t} \Theta(s) d s,
\end{aligned}
$$


and assume that

$$
\boldsymbol{E} \exp \left\{\frac{1}{2} \int_{0}^{T} \Theta^{2}(s) d s\right\}<\infty
$$

Set $Z=Z(T)$. Then $\boldsymbol{E} Z=1$ and under the probability measure $\tilde{\mathbb{P}}$ given by

$$
\tilde{\mathbb{P}}(A)=\int_{A} Z(\omega) d P(\omega) \text { for all } A \in \mathcal{F}
$$

the process $\tilde{W}(t)$ is a Brownian motion.

Note that by its construction $\tilde{\mathbb{P}}$ is equivalent to the initial measure $\mathbb{P}$. The proof of Girsanov's Theorem may be found in [28].

Assume that $\inf _{t, s} \sigma(t, s)>0$ and let us consider processes $\Theta(t)$ of a special form

$$
\tilde{\Theta}(t)=\frac{\mu(t, S(t))-r(t)}{\sigma(t, S(t))}
$$

then the measure $\tilde{\mathbb{P}}$ will be an equivalent martingale measure. Indeed, let us write the dynamics of the discounted stock process $S(t) / B(t)$

$$
d \frac{S(t)}{B(t)}=(\mu(t, S(t))-r(t)) \frac{S(t)}{B(t)} d t+\sigma(t, S(t)) \frac{S(t)}{B(t)} d W(t) .
$$

under $\tilde{\mathbb{P}}$. From theorem 2.6.1 the old Brownian motion $W(t)$ relates to the new $\tilde{W}(t)$ via

$$
d W(t)=d \tilde{W}(t)-\frac{\mu(t, S(t))-r(t)}{\sigma(t, S(t))} d t
$$

and thus

$$
d \frac{S(t)}{B(t)}=\sigma(t, S(t)) \frac{S(t)}{B(t)} d \tilde{W}(t)
$$

So the discounted stock price process is a martingale and $\tilde{\mathbb{P}}=\mathbb{Q}$ is an equivalent martingale measure.

Moreover, there are no other equivalent martingale measures in the generalized Black-Scholes model. We are going to use the converse Girsanov 2.6.2 and martingale representation 2.6.3 theorems for the proof of this fact. 
Theorem 2.6.2 (The Converse of the Girsanov, one dimension) Let $W(t)$ be a Brownian motion on $(\Omega, \mathcal{F}, P)$ with respect to filtration $\left\{\mathcal{F}_{t}\right\}_{t=0}^{T}$ generated by $W(t)$. Assume that there exists a probability measure $\mathbb{Q}$, such that $\mathbb{Q} \sim \mathbb{P}$ on $\mathcal{F}_{T}$. Then there exists an adapted process $\Theta$ such that if we define

$$
Z(t)=\exp \left\{-\int_{0}^{t} \Theta(s) d W(s)-\frac{1}{2} \int_{0}^{t} \Theta^{2}(s) d s\right\}
$$

then for all $A \in \mathcal{F}$

$$
\mathbb{Q}(A)=\int_{A} Z(T, \omega) d P(\omega) .
$$

The proof can be found in [8].

Theorem 2.6.3 (Martingale representation theorem) Let $W(t)$ be a Brownian motion on a probability space $(\Omega, \mathcal{F}, \mathbb{P})$, with respect to filtration $\left\{\mathcal{F}_{t}\right\}_{t=0}^{T}$ generated by $W(t)$. Let $M(t)$ be an $\mathcal{F}_{t}$-martingale process. Then there exists an adapted process $\Gamma(t)$ such that

$$
M(t)=M(0)+\int_{0}^{t} \Gamma(u) d W(u) .
$$

We refer the reader to [28] for the proof.

Let $\mathbb{Q}$ be an equivalent martingale measure. Then by the converse Girsanov theorem 2.6.2 there exists a measurable adapted square integrable process $\Theta(t)$ such that

$$
\tilde{W}(t)=W(t)+\int_{0}^{t} \Theta(s) d s
$$

is a Brownian motion under $\mathbb{Q}$. Thus we may write the dynamics for $\hat{S}(t)=$ $S(t) / B(t)$ under $\mathbb{Q}$ as

$$
d \hat{S}(t)=(\mu(t, S(t))-r(t)-\sigma(t, S(t)) \Theta(t)) \hat{S}(t) d t+\sigma(t, S(t)) \hat{S}(t) d \tilde{W}(t) .
$$

Since $\mathbb{Q}$ is a martingale measure and $\hat{S}(t)$ is a martingale then by theorem 2.6.3 we must have

$$
\Theta(t)=\frac{\mu(t, S(t))-r(t)}{\sigma(t, S(t))}
$$


as in (2.10). Therefore the equivalent martingale measure in the generalized Black-Scholes model is unique. So we may define the price process for an arbitrary contingent claim $X$ as the conditional expectation with respect to the risk-neutral measure $\mathbb{Q}$ of its discounted payoff:

$$
\Pi_{t}(X)=\mathbf{E}^{\mathbb{Q}}\left(\frac{B(t)}{B(T)} X \mid \mathcal{F}_{t}\right)
$$

for all $t \in[0, T]$. This price process is a martingale, therefore by the martingale representation theorem 2.6.3 it can be replicated. Thus we have proved that the generalized Black-Scholes model (2.2)-(2.3) is complete. And therefore any contingent claim in this model has a unique no-arbitrage price.

Note that the drift of the non-discounted stock process under the riskneutral measure is equal to the risk-free rate:

$$
d S(t)=r(t) S(t) d t+\sigma(t, S(t)) S(t) d \tilde{W}(t),
$$

which reflects the fact that the growth of a riskless portfolio is exactly the same as the growth of the bank account. This also explains the phenomenon we have noticed in section 2.4: the price of a simple contingent claim does not depend on the drift $\mu$ of the underlying asset $S(t)$.

We would like to consider an even more special case of the model in order to get an analytical formula for the price of the European Call. Let us assume, that all model parameters - the drift $\mu$, the volatility $\sigma$ and the interest rate $r$ are constants. This is the classical Black-Scholes model, presented in Black and Scholes [9] and Merton [33]. The underlying asset has the following dynamics under $\mathbb{Q}$ :

$$
d S(t)=r S(t) d t+\sigma S(t) d \tilde{W}(t) .
$$

Therefore at the maturity date of the option under $\mathbb{Q}$ conditionally on $\mathcal{F}_{t}$ the underlying asset price has the following form:

$$
S(T)=S(t) \exp \left\{\left(r-\frac{1}{2} \sigma^{2}\right)(T-t)+Y \sigma \sqrt{T-t}\right\},
$$

where $Y$ is a standard normal random variable under $\mathbb{Q}$. Now using proposition 2.5.1, we calculate the unique price of the Call option as

$$
\begin{aligned}
C(t, S(t)) & =\Pi_{t}(\max (S(T)-K, 0)) \\
& =\mathbf{E}^{\mathbb{Q}}\left(e^{-r(T-t)} \max (S(T)-K, 0) \mid \mathcal{F}_{t}\right) \\
& =\frac{e^{-r(T-t)}}{\sqrt{2 \pi}} \int_{-\infty}^{+\infty} \max \left(e^{\left(r-\frac{1}{2} \sigma^{2}\right)(T-t)+z \sigma \sqrt{T-t}}-K, 0\right) e^{-\frac{z^{2}}{2}} d z
\end{aligned}
$$


Performing the integration we get the famous Black-Scholes formula for the European Call option:

$$
\begin{aligned}
C(t, S) & =S N\left(d_{1}\right)-K e^{-r(T-t)} N\left(d_{2}\right) \\
d_{1} & =\frac{\ln (S / K)+\left(r+\frac{1}{2} \sigma^{2}\right)(T-t)}{\sigma \sqrt{T-t}} \\
d_{2} & =d_{1}-\sigma \sqrt{T-t}
\end{aligned}
$$

where $N(x)$ is the standard normal distribution function. In the similar way one may derive the Black-Scholes formula for the European Put:

$$
P(t, S)=K e^{-r(T-t)} N\left(-d_{2}\right)-S N\left(-d_{1}\right) .
$$




\section{Chapter 3}

\section{Incomplete Models}

In chapter 2 we introduced the notion of a complete market model and the Black-Scholes model as its example. If the real market followed the assumptions of Black and Scholes all options could be hedged in a riskless way and their prices would satisfy the explicit Black-Scholes formula. The volatility parameter of the model is a feature of the underlying asset and is the same for options with different strikes and maturities. Then inverting the market prices of options on the same underlying as a function of volatility would produce one value of the parameter for all options. In the this chapter we show that in reality this is not the case and therefore the assumption of constant volatility is not realistic.

The market completeness assumption itself is also often violated. In general, it is not possible to find a self-financing replicating strategy for a given contingent claim. Instead, many different strategies, each of them less than perfect in terms of replication, usually exist. Hence an optimization procedure is needed in order to choose one hedging strategy from those nonreplicating ones. This chapter presents two approaches to defining the optimization problem, which differ in the way they deal with the unhedged risk. The quadratic hedging makes the risk as small as the incomplete model allows, consequently minimizing a possible profit as well. The utility approach maximizes the trader's profit while controlling the risk according to the risk preferences defined by a utility function.

The structure of the chapter is as follows. First we introduce the notion of an implied volatility smile. Then we consider optimization methods for hedging and pricing of options: the quadratic hedging and the utility approach. 


\subsection{Volatility Smiles}

Implied volatility is the volatility determined from market prices of European options by inverting the Black-Scholes formula (2.12) or (2.13). To be more precise, we say that at a given time $t$, the risk-free rate $r$, underlying asset value $S$, strike price $K$, maturity date $T$ and the market price of a European option $C$ market the implied volatility $\hat{\sigma}$ is the value of the parameter $\sigma$ that produces the market price $C^{\text {market }}$, when inserted in the Black-Scholes formula (2.12) for a European Call together with the rest of the parameters:

$$
C^{\text {market }}=\operatorname{BS}(t, S, K, T, r, \hat{\sigma}) \text {. }
$$

It is market practice to quote vanilla options not as their prices but as implied volatilities. Quoting volatilities rather than prices makes it easier to compare relative values of Calls and Puts across strikes and expirations. Since valuing an option is essentially linked to one's view of uncertainty associated with the future prices of the underlying asset, implied volatility is the most natural way of expressing it.

If the market were consistent with the Black-Scholes model (2.11) then the implied volatilities would be the same for all European options on the same underlying asset, since the volatility parameter $\sigma$ is constant in this model. However, after the market crash on the 19th of October 1987, implied volatilities of equity options started to exhibit strong variability across strikes and maturities (see, for example, [15]). Nowadays this phenomenon is observed in FX, equity, interest rate and other options. Figure 3.1 presents the implied volatilities for the DAX options plotted against their maturities and strike prices. The surface seen on this graph is the so-called implied volatility surface. As we can see, the implied volatility surface is far from being flat, thus contradicting the Black-Scholes model. Because of its shape the graph of implied volatilities against strikes for some fixed maturity date is usually referred to as an implied volatility smile or an implied volatility skew, see, for example figure 3.2. Most of other liquid options markets also demonstrate non-flat volatility surfaces.

This example shows that the assumptions of Black-Scholes are too restrictive to hold true in real world markets. All later option pricing models relax various of those assumptions in order to capture the phenomenon of volatility smiles in option prices they produce. See, for example, [15], [17], [34] or [24]. The model we will present in chapter 5 is also capable of producing implied volatility smiles. 


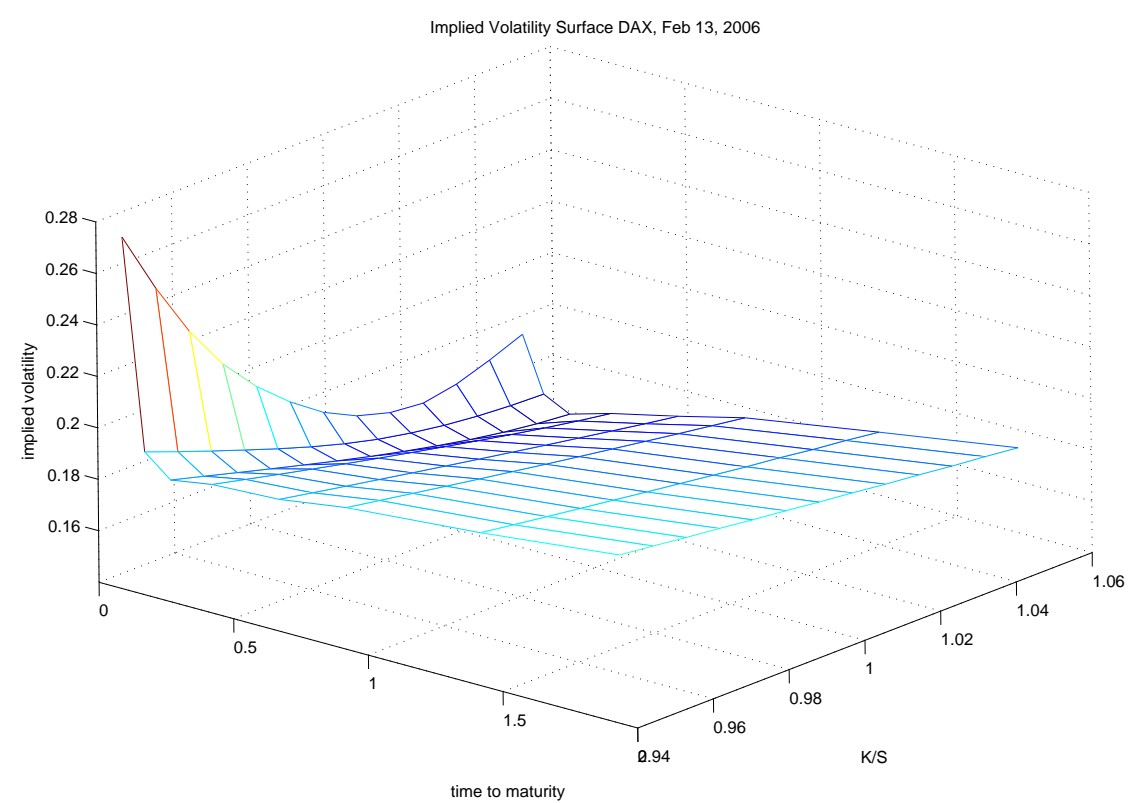

Figure 3.1: Volatility Surface for DAX on Feb 13, 2006.

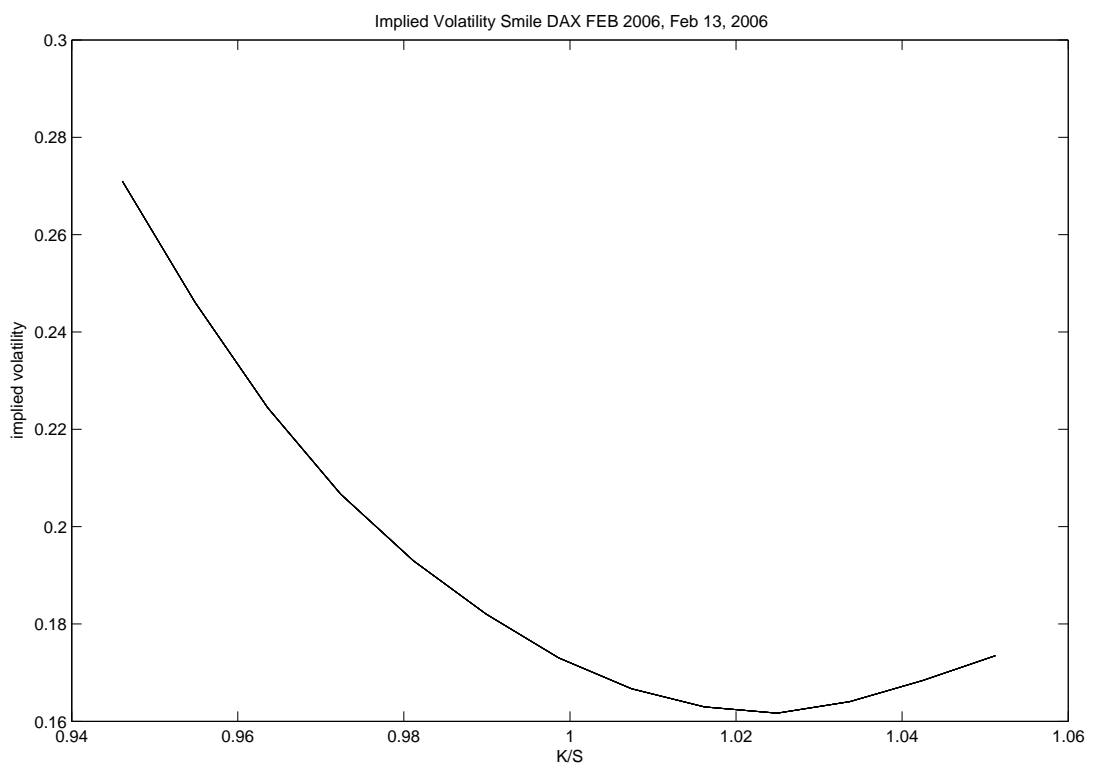

Figure 3.2: Volatility Smile for DAX FEB 2006 on Feb 13, 2006 


\subsection{Quadratic Hedging}

Quadratic hedging is a way to find an optimal hedging strategy in a general incomplete market setting by minimizing the expected squared hedging error or the expected squared hedging costs. The expectation of squared difference is the distance in $L^{2}$, thus minimization of the expected squared error is the minimization of this distance between the contingent claim and the trading strategy. In other words we look for the optimal strategy as an $L^{2}$-approximation of the contingent claim.

Through the rest of the section we follow the assumptions of Schweizer [45] and assume asset prices $S$ to be the discounted prices of the underlying assets. Then the riskless bank account is identically equal to 1 . Under the assumption of non-stochastic interest rates this does not affect the generality. If we denote by $\Theta$ the space

$$
\Theta=\left\{\theta \in L(S) \mid G(\theta) \in \mathcal{S}^{2}(\mathbb{P})\right\} .
$$

where $L(S)$ is the space of all $\mathbb{R}^{d}$-valued predictable processes integrable with respect to $S$ and $\mathcal{S}^{2}(\mathbb{P})$ is the space of square-integrable semimartingales. The total gains up to time $t$ from trade using the trading strategy $\theta$ is

$$
G_{t}(\theta):=\int_{0}^{t} \theta(u) d S(u)
$$

then $G_{T}(\Theta)$ is the set of all attainable payoffs.

The basic framework of quadratic hedging by means of a self-financing strategy may be described as follows. We look for a self-financing portfolio $\theta$ and initial capital $c \in \mathbb{R}$ that solve

$$
\min _{(c, \theta) \in \mathbb{R} \times \Theta} \mathbf{E}\left[X-c-G_{T}(\theta)\right]^{2}
$$

for a contingent claim $X$.

If non-self-financing portfolios are also allowed, then if $\theta=(\xi, \eta)$ is a hedging strategy, $\xi(t)$ - the number of stocks in the hedging portfolio and $\eta(t)$ - the amount of money in the bank account, $V(t)^{\theta}=\xi(t) S(t)+\eta(t)$ is the value process assumed to be square-integrable and $V^{\theta}(T)=X$, then the problem is to minimize the accumulated cost

$$
\begin{aligned}
R^{\theta}(t) & =\mathbf{E}\left[\left(C^{\theta}(T)-C^{\theta}(t)\right)^{2} \mid \mathcal{F}_{t}\right], \quad 0 \leq t \leq T, \\
C^{\theta}(t) & =V^{\theta}(t)-\int_{0}^{t} \xi(u) d S(u)
\end{aligned}
$$


in the following sense:

Definition 3.2.1 An admissible continuation of a trading strategy $\theta=$ $(\xi, \eta)$ from time $t \in[0, T)$ on is a trading strategy $\tilde{\theta}=(\tilde{\xi}, \tilde{\eta})$ satisfying

$$
\begin{aligned}
& \tilde{\xi}(s)=\xi(s), \quad \text { for } s \leq t, \\
& \tilde{\eta}(s)=\eta(s), \quad \text { for } s<t,
\end{aligned}
$$

and

$$
V^{\theta}(T)=V^{\tilde{\theta}}(T) \quad \mathbb{P}-\text { a.s. }
$$

The problem is to find a trading strategy $\theta$ such that for any $t \in[0, T)$ and any admissible continuation $\tilde{\theta}$ of $\theta$ from $t$ on

$$
R^{\tilde{\theta}}(t) \geq R^{\theta}(t) \quad \mathbb{P}-\text { a.s. }
$$

Such strategy is called $R$-minimizing.

Problems (3.1) and (3.3) are referred to as quadratic hedging. Problem (3.3) is considered in Schweizer [42], where he shows that for any contingent claim $X$ a unique $R$-minimizing strategy exists and it is mean-self-financing (e.g. its cost process is a martingale). Föllmer and Sonderman [18] were the first to approach the solution of (3.1). They considered a special case where $S$ is a martingale. Bouleau and Lamberton [10] have also restricted themselves to the martingale case while considering a Markovian formulation in order to get explicit solutions. The first attempt to extend the solution to the semimartingale case was made by Duffie and Richardson [16] for geometric Brownian motion and a particular case of hedging a non-traded asset with futures on another asset, correlated with the first one. Schweizer [41] generalizes this result to the hedging of an arbitrary contingent claim. For discrete time Schäl [40] considers the problem of cost minimization for the case of a constant investment opportunity set and gives conditions under which the price of the option does not depend on the choice of quadratic minimization criterion. Schweizer [44] also works in discrete time and solves the optimization problem for self-financing portfolios. Schweizer [43] and Monat and Stricker [35] work in continuous time but under strong restrictions on the underlying process. These two papers focus mainly on finding the optimal strategy $\theta^{(c)}$ for a given initial capital $c$ : given $X \in L^{2}(\mathbb{P})$ and $c \in \mathbb{R}$ solve

$$
\min _{\theta \in \Theta} \mathbf{E}\left[X-c-G_{T}(\theta)\right]^{2}
$$


while Schweizer [45] is also interested in computing the optimal $c$ :

$$
\min _{(c, \theta) \in \mathbb{R} \times \Theta} \mathbf{E}\left[X-c-G_{T}(\theta)\right]^{2} .
$$

It shows that the optimal initial capital $c$ is the expectation of the final payoff under a certain measure, which is called the variance-optimal measure.

Definition 3.2.2 A signed measure $\mathbb{Q}$ on $(\Omega, \mathcal{F})$ is called a signed $\Theta$-martingale measure if $\mathbb{Q}(\Omega)=1, \mathbb{Q}<<\mathbb{P}$ with $\frac{d \mathbb{Q}}{d \mathbb{P}} \in \mathcal{L}^{2}(\mathbb{P})$ and

$$
\boldsymbol{E}\left(\frac{d \mathbb{Q}}{d \mathbb{P}} G_{T}(\theta)\right)=0 \quad \text { for all } \theta \in \Theta .
$$

All signed $\Theta$-martingale measures are denoted by $\mathbb{P}_{s}(\Theta)$.A signed $\Theta$-martingale measure $\tilde{\mathbb{P}}$ is called variance-optimal if $\tilde{\mathbb{P}}$ solves

$$
\operatorname{Var}\left(\frac{d \tilde{\mathbb{P}}}{d \mathbb{P}}\right)=\min _{\mathbb{Q} \in \mathbb{P}_{s}(\Theta)} \operatorname{Var}\left(\frac{d \mathbb{Q}}{d \mathbb{P}}\right) .
$$

If $\mathbb{P}_{s}(\Theta)$ is not empty then $\tilde{P}$ exists and is unique since its density $\frac{d \tilde{\mathbb{P}}}{d \mathbb{P}}$ is obtained by minimizing $\left\|\frac{d \mathbb{Q}}{d \mathbb{P}}\right\|_{L^{2}}$ over the closed convex set $\left\{\frac{d \mathbb{Q}}{d \mathbb{P}} \mid \mathbb{Q} \in \mathbb{P}_{s}(\Theta)\right\}$. Schweizer [45] proves that if $(3.1)$ has a solution $(c, \theta)$ for $X \in L^{2}(\mathbb{P})$, then the optimal initial capital $c$ is an expectation of $X$ under the variance-optimal measure:

$$
c=\mathbf{E}^{\tilde{\mathbb{P}}}(X) .
$$

If the time set is discrete then the variance-optimal measure $\tilde{\mathbb{P}}$ may be explicitly constructed by backward recursion. Let $0=t_{0}<t_{1}<\ldots<t_{N}=T$ be the time set, then

$$
\frac{d \tilde{\mathbb{P}}}{d \mathbb{P}}=\frac{\tilde{Z}^{0}}{\mathbf{E}\left[\tilde{Z}^{0}\right]}
$$

where

$$
\tilde{Z}^{0}=\prod_{j=1}^{N}\left(1-\beta_{j} \Delta S_{j-1}\right)
$$

and

$$
\beta_{k}=\frac{\mathbf{E}\left[\Delta S_{k-1} \prod_{j=k+1}^{N}\left(1-\beta_{j} \Delta S_{j-1}\right) \mid \mathcal{F}_{k-1}\right]}{\mathbf{E}\left[\Delta S_{k-1}^{2} \prod_{j=k+1}^{N}\left(1-\beta_{j} \Delta S_{j-1}\right)^{2} \mid \mathcal{F}_{k-1}\right]}, \quad k=1, \ldots, N
$$


assuming that an empty product is equal to 1 . In chapter 4 we revisit this topic and consider the variance-optimal measure in the generalized BlackScholes setting.

In continuous time the variance-optimal measure can also be constructed via a continuous adjustment process $\beta$. If $\mathcal{E}_{t}^{\psi}$ denotes the stochastic exponential of $-\int_{0}^{t} \psi d S$ (or a solution of a stochastic differential equation $d Y(t)=-Y(t-) \psi(t) d S(t), Y(0)=1)$, then

$$
\frac{d \tilde{\mathbb{P}}}{d \mathbb{P}}=\frac{\tilde{Z}^{0}}{\mathbf{E}\left[\tilde{Z}^{0}\right]},
$$

where

$$
\tilde{Z}^{0}=\mathcal{E}_{T}^{\beta}
$$

where $\beta \in L(S)$ is such that $\beta \mathcal{E}_{-}^{\beta} \in \Theta$ and $\mathbf{E}\left[\mathcal{E}_{T}^{\beta} G_{T}(\theta)\right]=0$ for all $\theta \in \Theta$. Schweizer [45] shows that under the assumption of non-empty $\mathbb{P}_{s}(\Theta)$ process $\beta$ is unique in the following sense: all adjustment processes $\beta$ coincide on $\left\{\mathcal{E}_{-}^{\beta} \neq 0\right\} \subseteq \Omega \times[0, T]$ and $\left\{\mathcal{E}_{-}^{\beta} \neq 0\right\}$ does not depend on the choice of $\beta$. Under the same assumption the existence of the adjustment process $\beta$ is equivalent to the existence of a solution $(\beta, U) \in L(S) \times \mathcal{S}^{2}$ of the backward SDE

$$
\begin{aligned}
d U(t) & =-U(t-) \beta(t) d S(t), \\
U(T) & =\pi(1),
\end{aligned}
$$

where $\pi$ is $L^{2}(\mathbb{P})$-projection on $G_{T}(\Theta)$.

The following theorem gives the relation between the solutions of problems (3.1) and (3.4).

Theorem 3.2.1 (Schweizer, 1996) If $\mathbb{P}_{s}(\Theta)$ is not empty, $G_{T}(\Theta)$ is closed in $L^{2}(\mathbb{P}), X \in L^{2}(\mathbb{P})$ is fixed and $\theta^{(c)}$ denotes the solution of (3.4) for the initial capital $c$, then

1. $\left(\boldsymbol{E}^{\tilde{\mathbb{P}}}[X], \theta^{\left(\boldsymbol{E}^{\tilde{\mathbb{P}}}[X]\right)}\right)$ solves (3.4),

2. $\theta^{\left(\boldsymbol{E}^{\tilde{\tilde{T}}}[X]\right)}$ minimizes $\operatorname{Var}\left[X-G_{T}(\theta)\right]$ over all $\theta \in \Theta$,

3. if additionally $\boldsymbol{E}\left(\frac{d \tilde{\mathbb{P}}}{d \mathbb{P}}\right)^{2} \neq 1$ and $c_{m}$ denotes

$$
c_{m}=\frac{m \boldsymbol{E}\left[\frac{d \tilde{\mathbb{\mathbb { P }}}}{d \mathbb{P}}\right]^{2}-\boldsymbol{E}^{\tilde{\mathbb{P}}}[X]}{\boldsymbol{E}\left[\frac{d \tilde{\mathbb{P}}}{d \mathbb{P}}\right]^{2}-1},
$$


then $\theta^{\left(c_{m}\right)}$ is also the solution of

$$
\begin{aligned}
& \min _{\theta \in \Theta} \operatorname{Var}\left[X-G_{T}(\theta)\right] \\
& \text { s.t. } \boldsymbol{E}\left[X-G_{T}(\theta)\right]=m
\end{aligned}
$$

for a given $m \in \mathbb{R}$.

(Note that since $G_{T}(\Theta)$ is closed in $L^{2}(\mathbb{P})$ then (3.4) has a solution $\theta^{(c)}$ for any $c \in \mathbb{R}$.)

Gourieroux et al. [21] solve the same problem by introducing the hedging numeraire, which is a strictly positive value process of a self-financing hedging strategy. Using this process as a deflator and also as a traded asset, they give a simpler equivalent formulation of the optimization problem. Laurent and Pham [29] apply dynamic programming methods to the problem with numeraire and thus provide an explicit form for the value process and the variance-optimal martingale measure. Černý [47] revisits the discrete time case and extends Schäl [40] to a non-constant investment opportunity set and by means of dynamic programming gets a recursive procedure for optimization, which is very well suited for computer implementation. He also shows how the variance-optimal measure arises in the dynamic programming solution and how one defines conditional expectations under this (generally not equivalent) measure. We will come back to this topic in chapter 4 and derive some properties of the optimal hedging strategies and the variance-optimal measure in the generalized Black-Scholes model.

Theoretically finding the trading strategy, which is the closest (in some sense) to the payoff, is a natural extension of the complete market theory, where the distance can be minimized to zero. Nevertheless the minimization of the expected squared error minimizes not only the risk but also the profit. This fact makes the quadratic hedging model not very realistic as the trader's goal is the maximization of profit under certain risk constraints. A way to address this problem is the utility maximization, which we discuss in the following section.

\subsection{Utility Functions and Indifference Pric- ing}

Another way to find an optimal hedging strategy is the maximization of the expected utility of profit. Under the profit we understand either the total consumption if consumption is non-zero or the final payoff if the portfolio is self-financing or combination of both. 
According to a general economic theory, utility is a measure of the relative satisfaction from getting a certain good. It is described by a utility function $U: G \rightarrow \mathbb{R}$, where $G$ is the set of available goods. The utility function allows the consumer to rank $G$ according to his or her preferences:

$$
g_{1} \prec g_{2} \Leftrightarrow U\left(g_{1}\right)<U\left(g_{2}\right) .
$$

Marginal utility is the increase in the utility obtained by consuming a unit of a good. The law of diminishing marginal utility first proposed in Bernoulli [5] states that the marginal utility decreases with the increase of the consumption of a certain good. So each additional unit will increase the utility less than the ones before it. In other words, the more we get of something the less we value each additional unit of it.

In mathematical finance all goods are money, which corresponds to setting $G=\mathbb{R}$ and a continuous monotonously increasing concave utility function $U: \mathbb{R} \rightarrow \mathbb{R}$. Note that since $U$ is monotonous it keeps the relation on the real numbers. For all $x_{1}, x_{2} \in \mathbb{R}$

$$
x_{1} \prec x_{2} \Leftrightarrow U\left(x_{1}\right)<U\left(x_{2}\right) \Leftrightarrow x_{1}<x_{2} .
$$

The marginal utility is then the derivative $U^{\prime}$ of the utility function and the concavity ensures that it decreases and therefore the law of diminishing marginal utility holds.

The maximization of expected utility in finance was first used for the consumption-portfolio optimization, where the problem is to maximize the expected utility of the portfolio consumption:

$$
\max \int_{0}^{T} e^{-r t} U(C(t)) d t,
$$

or

$$
\max \sum_{k=1}^{N} e^{-r t_{k}} U\left(C\left(t_{k}\right)\right)
$$

for the discrete time case, where $C(t)$ is the portfolio consumption per unit of time. Markowitz [30] solved the problem for a one period model, Samuelson [39] generalized the solution to the multi-period case and Merton [32] formulated the continuous time version for the portfolio of assets with dynamics following the geometric Brownian motions. The same paper gives an explicit solution for the special case of utility functions such that

$$
-\frac{U^{\prime \prime}}{U^{\prime}}(x)=\frac{1}{\frac{x}{1-\gamma}+\frac{\eta}{\beta}},
$$


for some $\gamma>1, \beta>0, \eta=1$ if $\gamma=-\infty$ and

$$
\frac{\beta x}{1-\gamma}+\eta>0
$$

the so called hyperbolic absolute risk-aversion (or HARA) utility functions.

Note that the mean-variance hedging problem (3.4) from section 3.2 can be reformulated in terms of quadratic utility maximization

$$
\max _{\theta \in \Theta} \mathbf{E}\left[\tilde{U}\left(X-G_{T}(\theta)\right)\right]
$$

with

$$
\tilde{U}(x)=x-\frac{1}{2 c} x^{2} .
$$

Nevertheless the utility theory cannot be fully applied to the expected squared error minimization problem as $\tilde{U}$ defined in (3.8) monotonously increases only for $x<c$.

Basak and Shapiro [2] provides analytical solutions for the expected utility maximization under a constraint on the trader's Value-at-Risk (VaR) and under a Limited-Expected-Loss (LEL) constraint. Value-at-Risk of a portfolio $\theta$ is the loss, which is exceeded with some given probability $\alpha$ over a given horizon (see, for example [27]):

$$
\mathbb{P}\left[V^{\theta}(0)-V^{\theta}(T) \leq \operatorname{VaR}(\alpha)\right]=1-\alpha, \quad 0 \leq \alpha \leq 1
$$

The constraint on VaR may be written as

$$
\mathbb{P}\left[V^{\theta}(T) \geq F\right] \geq 1-\alpha
$$

for some exogenously defined "floor" F. Basak and Shapiro [2] argues that since a trader with constraint (3.9) is concerned with controlling the probability of a loss rather then its magnitude the expected losses may be higher than those of an unconstrained trader. They propose a LEL-risk management instead with a constraint

$$
\mathbf{E}^{\mathbb{Q}}\left[F-V^{\theta}(T) \mathbf{1}_{\left\{V^{\theta}(T) \leq F\right\}}\right] \leq \epsilon
$$

for some constant $\epsilon \geq 0$. The strategies optimal under this constraint show smaller losses then those of an unconstrained trader.

Another application of the expected utility maximization is the hedging of options in the presence of transaction costs. Transaction costs make the 
continuous trading infinitely expensive when asset prices have infinite variation and thus perfect replication is impossible. The utility based approach for proportional transaction costs was first proposed by Hodges and Neuberger [25]. They solve the problem

$$
\begin{aligned}
J^{\Phi}\left(0, S_{0}, x_{0}, y_{0}\right) & =\max \mathbf{E}\left[U\left(V_{T}\right)\right] \\
V_{T} & =x_{T} S_{T}-k\left(x_{T}, S_{T}\right)+y_{T}-\Phi\left(S_{T}\right),
\end{aligned}
$$

where $\Phi(\cdot)$ is the option's payoff $k(x, S)$ is the transaction fee for selling $x$ stocks for the price $S, x_{t}$ is the number of stocks and $y_{t}$ is the amount of cash in the hedging portfolio at time $t$. Davis and Norman [12] have formulated the utility maximization as a singular stochastic control problem and Davis et al. [13] have proved that the problem of Hodges and Neuberger amounts to the problem of Davis and Norman with singular control.

Hodges and Neuberger [25] were the first to introduce the notion of indifference prices - the amount of money which makes the trader indifferent between (1) selling or buying an option and then optimize utility and (2) maximizing his or her utility without an option. If $J^{\Phi}\left(0, S_{0}, x_{0}, y_{0}\right)$ is the solution of (3.10) for payoff $\Phi$, then the indifference selling price $p^{s}$ is defined by

$$
J^{\Phi}\left(0, S, 0, p^{s}\right)=J^{0}(0, S, 0,0)
$$

and the indifference buying price $p^{b}$ by

$$
J^{-\Phi}\left(0, S, 0,-p^{b}\right)=J^{0}(0, S, 0,0) .
$$

So the indifference price is the initial capital that results in the same optimal value function for the option buyer or seller as the zero initial capital for the trader in the underlying market. Davis et al. [13] also define the indifference prices for their model in a similar way. They prove that if a replicating portfolio exists, then the indifference price is equal to the price of the replicating portfolio. Stoikov [46] introduces the notion of the relative indifference price - an amount of money which makes the trader indifferent between hedging a portfolio of options and hedging this portfolio of options plus the option to be priced. This model allows the trader to quote competitive prices that depend on his or her portfolio.

Musiela and Zariphopoulou [36] find explicit solutions for the indifference price of a European claim $G$ under the assumptions of lognormal dynamics of the underlying assets and exponential utility function $U(x)=-\exp (-\gamma x)$ for some $\gamma>0$. The underlying asset of the claim $G$ is assumed to be 
non-traded, its observed level follows the dynamics given by:

$$
\begin{aligned}
d Y(s) & =b(s, Y(s)) d s+a(s, Y(s)) d W^{1}(S), \quad s \geq t \\
Y(t) & =y
\end{aligned}
$$

for some $y \in \mathbb{R}$. It assumes the existence of a riskless bank account $B$. Without the loss of generality the risk-free rate $r=0$. The trader's goal is to maximize his or her utility at time $T$ by trading $B$ and a risky traded asset $S$ correlated with $Y$. $S$ follows the Black-Scholes dynamics

$$
\begin{aligned}
d S(s) & =\mu S(s) d s+\sigma S(s) d W^{2}(s), \quad s \geq t \\
S(t) & =S
\end{aligned}
$$

with $S>0$ and $\left\langle d W^{1}(t), d W^{2}(t)\right\rangle=\rho d t$. Under the assumptions above [36] shows that the indifference selling price of $G=g(Y(T))$ is a non-linear functional of the option's payoff:

$$
h(t, y)=\frac{1}{\gamma\left(1-\rho^{2}\right)} \ln \mathbf{E}^{\tilde{\mathbb{Q}}}\left[e^{\gamma\left(1-\rho^{2}\right) g(Y(T))} \mid Y(t)=y\right],
$$

where the measure $\tilde{\mathbb{Q}}$ is defined by

$$
\tilde{\mathbb{Q}}(A)=\mathbf{E}^{\mathbb{P}}\left[\exp \left\{-\frac{\mu}{\sigma} W^{2}(T)-\frac{1}{2} \frac{\mu^{2}}{\sigma^{2}} T\right\} \mathbf{1}_{A}\right], \quad A \in \mathcal{F}_{T} .
$$

The utility theory is a way to combine the profit maximization and the risk management according to the trader's risk preferences. Unfortunately practitioners do not like using utility functions as in most cases it is impossible to determine the utility function of a trader (see [11]). In chapter 5 we propose a model, in which the risk management and the risk preferences are modeled without utility functions, directly in terms of observable market parameters and the trader's current position in options. We define relative indifference prices of a portfolio of European options dependent on current traders' portfolios. 


\section{Chapter 4}

\section{Mean-Variance Hedging for Black-Scholes Dynamics}

One of the possible reasons for market incompleteness is the impossibility of continuous trading. This chapter is dedicated to a model which reflects this feature. The discrete dates at which trades may be performed are fixed and the expectation of the squared difference between the terminal values of the hedging portfolio and the option payoff is minimized. This brings us to the mean-variance hedging of Schweizer [45] which we described in chapter 3. As in Černý [47] we apply the dynamic programming method to the minimization. This allows us to get recursive formulae for the optimal strategy and the optimal initial capital. We prove that the cost function is a quadratic function of the portfolio value, which makes the recursive formulae simpler than those of Cerný [47].

Under the assumption of independent returns the analytic formula for the variance-optimal measure immediately follows. We prove that the RadonNikodym derivative of this measure with respect to the real-world measure converges in $L^{2}$ to the Radon-Nikodym derivative of the risk-neutral measure of the limiting complete model as the distance between the hedging dates goes to zero. Mercurio and Vorst [31] also consider convergence properties of the model but include only the convergence of European options prices but not measures. Černý [47] presents a heuristic proof of weak convergence of variance-optimal measure to the risk-neutral measure.

\subsection{Formulation of Hedging Problem}

We consider a discrete-time market model. The set of time points $\mathcal{T}=\left\{0=t_{0}<t_{1}<\ldots<t_{N}=T\right\}$ represents the fixed hedging dates 
and the maturity date with $\Delta t_{k}=t_{k+1}-t_{k}(k=0, \ldots, N-1)$, the time periods between successive hedges. Through the rest of this thesis we use the following notation:

$$
\begin{aligned}
X_{k} & =X\left(t_{k}\right), \quad k=0, \ldots, N \\
\Delta X_{k} & =X_{k+1}-X_{k}, \quad k=0, \ldots, N-1
\end{aligned}
$$

for any process $X$, in other words we work with forward differences. We assume that our market consists of $m$ risky assets $S^{1}, \ldots, S^{m}: \Omega \times \mathcal{T} \rightarrow \mathbb{R}^{+}$ and a riskless bank account $B: \mathcal{T} \rightarrow \mathbb{R}^{+}$with the following dynamics

$$
\left\{\begin{array}{l}
B_{k}=e^{r t_{k}} \\
S_{k+1}^{i}=S_{k}^{i} R_{k}^{i}, i=1, \ldots, m, k=0, \ldots, N-1,
\end{array}\right.
$$

where $\vec{S}_{0}=\left(S_{0}^{1}, \ldots, S_{0}^{m}\right)$ and $\vec{R}_{k}=\left(R_{k}^{1}, \ldots, R_{k}^{m}\right), k=1, \ldots N-1$ are $m$ dimensional random vectors. The corresponding filtration is $\left\{\mathcal{F}_{k}\right\}_{k=0}^{N}, \mathcal{F}_{k}=$ $\sigma\left(\left\{\vec{S}_{0}, \vec{R}_{0}, \ldots, \vec{R}_{k-1}\right\}\right), k=0, \ldots, N$. We assume that all assets are linearly independent.

The trader has to hedge a simple $T$-contingent claim with payoff $\Phi\left(S_{N}^{1}, \ldots, S_{N}^{m}\right)$, where $\Phi: \mathbb{R}^{m} \rightarrow \mathbb{R}$ is a measurable function. A hedging portfolio (or a hedging strategy) is a pair of adapted processes $\{\phi, \psi\}$, where $\phi: \Omega \times \mathcal{T} \backslash\left\{t_{N}\right\} \rightarrow \mathbb{R}^{m}$ represents the numbers of underlying assets and $\psi: \Omega \times \mathcal{T} \backslash\left\{t_{N}\right\} \rightarrow \mathbb{R}$ the number of bonds in the account $B$. Note that the portfolio process is not defined at the maturity date because there is no hedging at maturity. We denote the value of the hedging portfolio by $V$ :

$$
\begin{aligned}
V_{k} & =\left(\phi_{k}\right)^{T} S_{k}+\psi_{k} B_{k}, \quad k=0, \ldots, N-1, \\
V_{N} & =\left(\phi_{N-1}\right)^{T} S_{N}+\psi_{N-1} B_{N} .
\end{aligned}
$$

Definition 4.1.1 The set of admitted trading strategies $\mathcal{A}$ consists of all self-financing strategies such that the changes in the portfolio value may happen only due to the changes in the traded assets prices.

$$
\mathcal{A}=\left\{(\phi, \psi) \mid \Delta\left(\left(\phi_{k}\right)^{T} S_{k}+\psi_{k} B_{k}\right)=\left(\phi_{k}\right)^{T} \Delta S_{k}+\psi_{k} \Delta B_{k} \forall k=0, \ldots, N-1\right\}
$$

The trader's goal is to minimize the expected squared difference between the terminal value of the hedging portfolio $V_{N}$ and the option payoff $\Phi\left(S_{N}^{1}, \ldots, S_{N}^{m}\right)$ over all admitted strategies:

$$
\min _{(\phi, \psi) \in \mathcal{A}} \mathbf{E}\left[V_{N}-\Phi\left(S_{N}^{1}, \ldots, S_{N}^{m}\right)\right]^{2}
$$


If strategy $(\phi, \psi)$ is admitted, then for all $k=0, \ldots, N-1$

$$
\begin{aligned}
V_{k+1} & =V_{k}+\left(\phi_{k}\right)^{T} \Delta S_{k}+\psi_{k} \Delta B_{k} \\
& =V_{k}+\left(\phi_{k}\right)^{T}\left(\operatorname{diag}\left(R_{k}\right)-I\right) S_{k}+\psi_{k} B_{k}\left(e^{r \Delta t_{k}}-1\right),
\end{aligned}
$$

where $\operatorname{diag}\left(R_{k}\right)$ is a diagonal $m \times m$ matrix $d$ with diagonal elements $d^{i i}=R_{k}^{i}$ for $i=1, \ldots, m$ and $I$ is the unit matrix of dimension $m$. From the definition of the portfolio value $V_{k}$ in (4.2) we derive that

$$
\psi_{k} B_{k}=V_{k}-\left(\phi_{k}\right)^{T} S_{k},
$$

which we substitute in (4.4) to get

$$
V_{k+1}=V_{k}+\left(\phi_{k}\right)^{T}\left(\operatorname{diag}\left(R_{k}\right)-I\right) S_{k}+\left(V_{k}-\left(\phi_{k}\right)^{T} S_{k}\right)\left(e^{r \Delta t_{k}}-1\right) .
$$

Hence if the strategy is admitted then the portfolio value dynamics may be written as

$$
V_{k+1}=V_{k} e^{r \Delta t_{k}}+\left(\phi_{k}\right)^{T}\left(\operatorname{diag}\left(R_{k}\right)-e^{r \Delta t_{k}} I\right) S_{k} .
$$

Let us formulate optimization problem (4.3) in terms of a dynamic programming problem. Note, that for any adapted process $\phi$ there exists a unique adapted process $\psi$ (defined by 4.5 ) such that the strategy $(\phi, \psi)$ is admitted. Therefore the optimization over all admitted strategies $(\phi, \psi)$ is equivalent to the optimization over all adapted processes $\phi$.

\subsection{Dynamic Programming Problem}

First let us recall some general definitions and the theorem describing the dynamic programming method. Consider a discrete-time dynamic system

$$
x_{k+1}=h_{k}\left(x_{k}, u_{k}, w_{k}\right), \quad k=0,1, \ldots, N-1,
$$

where $x_{k} \in M_{k}$, the state space, the control $u_{k} \in G_{k}$, the control space, and the random disturbance $w_{k} \in H_{k}, k=0,1, \ldots, N-1$. The control $u_{k}$ is constrained by $u_{k} \in U_{k}\left(x_{k}\right) \subset G_{k},(k=0,1, \ldots, N-1)$ for given mappings $U_{k}: M_{k} \rightarrow 2^{G_{k}}$. We consider the class of control laws ("policies") that consist of a finite sequence of functions $\pi=\left\{\mu_{0}, \mu_{1}, \ldots, \mu_{N-1}\right\}$, where $\mu_{k}: M_{k} \rightarrow G_{k}$ and $\mu_{k}\left(x_{k}\right) \in U_{k}\left(x_{k}\right)$. Such control laws are called admitted.

The problem is to find an admitted control law $\pi=\left\{\mu_{0}, \ldots, \mu_{N-1}\right\}$ that for a given initial state $x_{0}$ minimizes the cost functional

$$
\begin{aligned}
J^{\pi}\left(x_{0}\right) & =\mathbf{E}\left[g_{N}\left(x_{N}\right)+\sum_{k=0}^{N-1} g_{k}\left(x_{k}, \mu_{k}\left(x_{k}\right), w_{k}\right)\right] \\
x_{k+1} & =h_{k}\left(x_{k}, \mu_{k}\left(x_{k}\right), w_{k}\right) .
\end{aligned}
$$


The functions $g_{N}: M_{N} \rightarrow \mathbb{R}, \quad g_{k}: M_{k} \times G_{k} \times H_{k} \rightarrow \mathbb{R}$ and $h_{k}: M_{k} \times G_{k} \times$ $H_{k} \rightarrow \mathbb{R}$ are assumed to be given, for $k=0,1, \ldots, N-1$.

Theorem 4.2.1 (Dynamic Programming) Let $J^{*}\left(x_{0}\right)$ be the optimal value of the cost functional (4.6) in the dynamic programming problem. Then

$$
J^{*}\left(x_{0}\right)=J_{0}\left(x_{0}\right),
$$

where the function $J_{0}$ is given by the last step of the following dynamic programming algorithm, which proceeds backward in time from period $N-1$ to period 0:

$$
\begin{aligned}
J_{N}\left(x_{N}\right)= & g_{N}\left(x_{N}\right) \\
J_{k}\left(x_{k}\right)= & \inf _{u_{k} \in U_{k}\left(x_{k}\right)} \boldsymbol{E}_{w_{k}}\left[g_{k}\left(x_{k}, u_{k}, w_{k}\right)+J_{k+1}\left(h_{k}\left(x_{k}, u_{k}, w_{k}\right)\right)\right] \\
& k=0,1, \ldots, N-1,
\end{aligned}
$$

where $\boldsymbol{E}_{w_{k}}$ is the expectation with respect to the distribution of $w_{k}$. Furthermore, if $u_{k}^{*}=\mu_{k}^{*}\left(x_{k}\right)$ minimizes the right-hand side of (4.7) for each $x_{k}$ and $k$ then the control law $\pi=\left\{\mu_{0}^{*}, \ldots, \mu_{N-1}^{*}\right\}$ is optimal.

The proof can be found in Bagchi [1] or Bertsekas [6]. Note that the above formulation of dynamic programming optimizes the cost function over Markov policies $\pi$ only. Nevertheless taking non-Markov policies into consideration does not lead to the reduction of the cost function in a model with a Markov underlying process. This fact is proved in Bertsekas and Shreve [7] for a slightly different cost function, but can be extended to the case of the cost function defined as above.

We can now state the dynamic program for the optimization problem (4.3). Our state variable is the pair $(S, V)$ - the price of the underlying vector $S \in \mathbb{R}^{m}$ and the value of the hedging portfolio $V \in \mathbb{R}$. The control variable is the number of risky assets $\phi \in \mathbb{R}^{m}$. The random disturbances are $w_{k}=R_{k}$. And the expectation with respect to the distribution of $R_{k}$ in (4.7) is equal to the conditional expectation with respect to $\mathcal{F}_{k}$. Further on for the sake of brevity we will write $\mathbf{E}_{k}$ instead of $\mathbf{E}\left[\cdot \mid \mathcal{F}_{k}\right]$. The dynamics of the state variables are given by

$$
\begin{aligned}
& S_{k+1}=\operatorname{diag}\left(R_{k}\right) S_{k} \\
& V_{k+1}=V_{k} e^{r \Delta t_{k}}+\left(\phi_{k}\right)^{T}\left(\operatorname{diag}\left(R_{k}\right)-e^{r \Delta t_{k}} I\right) S_{k},
\end{aligned}
$$

for all $k=0, \ldots, N-1$, for a given $\mathcal{F}_{0}$-measurable $\left(S_{0}, V_{0}\right) \in \mathbb{R}^{m} \times \mathbb{R}$. The control $\phi$ is unconstrained. $g_{k}=0$ for all $k<N$ and our cost function is given by

$$
J\left(S_{0}, V_{0}\right)=\mathbf{E}\left[\left(\Phi\left(S_{N}\right)-V_{N}\right)^{2}\right]
$$


and the minimization problem is

$$
\min _{\phi_{0}, \ldots, \phi_{N-1}} J\left(S_{0}, V_{0}\right) .
$$

We define the following dynamic programming algorithm for the hedging problem:

$$
\begin{aligned}
& J_{N}(S, V)=(\Phi(S)-V)^{2} \\
& J_{k}(S, V)=\inf _{\phi_{k}} \mathbf{E}_{k}\left[J_{k+1}\left(\operatorname{diag}\left(R_{k}\right) S, V e^{r \Delta t_{k}}+\phi_{k}^{T}\left(\operatorname{diag}\left(R_{k}\right)-e^{r \Delta t_{k}} I\right) S\right)\right], \\
& k=0, \ldots, N-1 .
\end{aligned}
$$

Then by Theorem 4.2.1 $J_{0}\left(S_{0}, V_{0}\right)$ is the minimal expected squared error for the initial wealth $V_{0}$ and initial asset values $S_{0}$. The optimal control policy $\phi_{0}^{*}\left(S_{k}, V_{k}\right), \ldots, \phi_{N-1}^{*}\left(S_{k}, V_{k}\right)$ defines the optimal hedging strategy.

\subsection{Properties of the Cost Function and the Recursive Algorithm}

Let us denote by $Y_{k}: \Omega \rightarrow \mathbb{R}^{m}$ the following random variable:

$$
\begin{aligned}
Y_{k} & =R_{k}-e^{r \Delta t_{k}} \vec{e} \\
\vec{e} & =(1, \ldots, 1)^{T} \in \mathbb{R}^{m}
\end{aligned}
$$

then the dynamic program (4.8) is

$$
\begin{aligned}
& J_{N}(S, V)=(\Phi(S)-V)^{2} \\
& J_{k}(S, V)=\inf _{\phi_{k}} \mathbf{E}_{k}\left[J_{k+1}\left(\operatorname{diag}\left(R_{k}\right) S, V e^{r \Delta t_{k}}+\phi_{k}^{T} \operatorname{diag}\left(Y_{k}\right) S\right)\right], \\
& k=0, \ldots, N-1 .
\end{aligned}
$$

The following proposition states that the cost functions $J_{k}(S, V), k=0, \ldots, N$ are quadratic functions of $V$. Its proof includes formulae for explicit recursion for $J_{k}(S, V), k=0, \ldots, N$ and the optimal control $\phi_{k}^{*}(S, V), k=0, \ldots, N-1$ for all $S \in \mathbb{R}^{+}$and $V \in \mathbb{R}$. Although obtained independently this proposition repeats the result of [22]. Unfortunately we have not come across this paper earlier.

Proposition 4.3.1 The cost functions $J_{k}(S, V)$, defined in (4.8) are quadratic functions of $V$ of the following form:

$$
J_{k}(S, V)=A_{k} V^{2}+C_{k}(S) V+D_{k}(S)
$$

for all $k=0, \ldots, N, S \in \mathbb{R}^{m}, V \in \mathbb{R}, A_{k}>0, C_{k}: \mathbb{R}^{m} \rightarrow \mathbb{R}$ and $D_{k}: \mathbb{R}^{m} \rightarrow$ $\mathbb{R}$, where numbers $A_{k}$ and functions $C_{k}$ and $D_{k}$ do not depend on $V$. 
Proof We prove this proposition by backward induction in $k$.

1. $k=N$.

$$
J_{N}=(\Phi(S)-V)^{2},
$$

therefore

$$
\begin{aligned}
A_{N} & =1>0 \\
C_{N}(S) & =-2 \Phi(S) \\
D_{N}(S) & =\Phi^{2}(S) .
\end{aligned}
$$

2. Assume the statement of the proposition is proved for all $k+1, \ldots, N$. Let us prove it for $k$. From (4.8)

$$
J_{k}(S, V)=\inf _{\phi_{k}} \mathbf{E}_{k}\left[J_{k+1}\left(\operatorname{diag}\left(R_{k}\right) S, V e^{r \Delta t_{k}}+\phi_{k}^{T} \operatorname{diag}\left(Y_{k}\right) S\right)\right] .
$$

By the induction assumption $J_{k+1}(S, V)$ is quadratic function of $V$, therefore

$$
\begin{aligned}
J_{k}(S, V)=\inf _{\phi_{k}} & \mathbf{E}_{k}\left[A_{k+1}\left(V e^{r \Delta t_{k}}+\phi_{k}^{T} \operatorname{diag}\left(Y_{k}\right) S\right)^{2}\right. \\
& +C_{k+1}\left(\operatorname{diag}\left(R_{k}\right) S\right)\left(V e^{r \Delta t_{k}}+\phi_{k}^{T} \operatorname{diag}\left(Y_{k}\right) S\right) \\
& \left.+D_{k+1}\left(\operatorname{diag}\left(R_{k}\right) S\right)\right],
\end{aligned}
$$

taking the expectation inside we get

$$
\begin{aligned}
J_{k}(S, V)=\inf _{\phi_{k}} & \left\{A _ { k + 1 } \left(\phi_{k}^{T} \mathbf{E}_{k}\left[\operatorname{diag}\left(Y_{k}\right) S S^{T} \operatorname{diag}\left(Y_{k}\right)\right] \phi_{k}+V^{2} e^{2 r \Delta t_{k}}\right.\right. \\
& \left.+2 V e^{r \Delta t_{k}} \phi_{k}^{T} \mathbf{E}_{k}\left[\operatorname{diag}\left(Y_{k}\right)\right] S\right) \\
& +\mathbf{E}_{k}\left[C_{k+1}\left(\operatorname{diag}\left(R_{k}\right) S\right)\right] V e^{r \Delta t_{k}} \\
& +\phi_{k}^{T} \mathbf{E}_{k}\left[C_{k+1}\left(\operatorname{diag}\left(R_{k}\right) S\right) \operatorname{diag}\left(Y_{k}\right)\right] S \\
& \left.+\mathbf{E}_{k}\left[D_{k+1}\left(\operatorname{diag}\left(R_{k}\right) S\right)\right]\right\}
\end{aligned}
$$

which is a quadratic function of $V$.

In the rest of the proof we derive the explicit recursion for the coefficients $A_{k}, C_{k}, D_{k}, k=0, \ldots, N-1$. And prove that $A_{k}>0$ for all $k=0, \ldots, N$. For all $S \in \mathbb{R}^{+}$and $V \in \mathbb{R}, J_{k}(S, V)$ is the minimum of a quadratic function of $\phi_{k}$. Its highest order coefficient $\mathbf{E}_{k}\left[\operatorname{diag}\left(Y_{k}\right) S S^{T} \operatorname{diag}\left(Y_{k}\right)\right]$ is the Gramian matrix in the space of random variables with scalar product defined as

$$
\langle X, Y\rangle=\mathbf{E}_{k}[X Y]
$$

for vector $\operatorname{diag}\left(Y_{k}\right) S$ which coordinates are linearly independent by the model assumptions. Therefore $\mathbf{E}_{k}\left[\operatorname{diag}\left(Y_{k}\right) S S^{T} \operatorname{diag}\left(Y_{k}\right)\right]$ is positive definite and 
hence for all $S \in \mathbb{R}^{+}$and $V \in \mathbb{R}$ there is a unique optimal $\phi_{k}^{*}(S, V)$, solving (4.10). Due to the quadratic structure of (4.10) $\phi_{k}^{*}$ is the solution to the following linear equation:

$$
\begin{aligned}
2 A_{k+1} \mathbf{E}_{k}\left[\operatorname{diag}\left(Y_{k}\right) S S^{T} \operatorname{diag}\left(Y_{k}\right)\right] \phi_{k}^{*} & +\mathbf{E}_{k}\left[C_{k+1}\left(\operatorname{diag}\left(R_{k}\right) S\right) \operatorname{diag}\left(Y_{k}\right)\right] S \\
+2 A_{k+1} V e^{r \Delta t_{k}} \mathbf{E}_{k}\left[\operatorname{diag}\left(Y_{k}\right)\right] S & =0
\end{aligned}
$$

Note that

$$
\operatorname{diag}\left(Y_{k}\right) S=\operatorname{diag}(S) Y_{k}
$$

hence we can rewrite (4.11) as

$$
\begin{aligned}
2 A_{k+1} \operatorname{diag}(S) \mathbf{E}_{k}\left[Y_{k} Y_{k}^{T}\right] \operatorname{diag}(S) \phi_{k}^{*} & +\operatorname{diag}(S) \mathbf{E}_{k}\left[C_{k+1}\left(\operatorname{diag}\left(R_{k}\right) S\right) Y_{k}\right] \\
+2 A_{k+1} V e^{r \Delta t_{k}} \operatorname{diag}(S) \mathbf{E}_{k}\left[Y_{k}\right] & =0
\end{aligned}
$$

If we multiply (4.13) by $(\operatorname{diag}(S))^{-1}$ on the left we get:

$$
\begin{aligned}
2 A_{k+1} \mathbf{E}_{k}\left[Y_{k} Y_{k}^{T}\right] \operatorname{diag}(S) \phi_{k}^{*} & +\mathbf{E}_{k}\left[C_{k+1}\left(\operatorname{diag}\left(R_{k}\right) S\right) Y_{k}\right] \\
& +2 A_{k+1} V e^{r \Delta t_{k}} \mathbf{E}_{k}\left[Y_{k}\right]=0,
\end{aligned}
$$

therefore for all $S \in \mathbb{R}^{+}$and $V \in \mathbb{R}$ the optimal $\phi_{k}^{*}(S, V)$ satisfies

$$
\begin{gathered}
\operatorname{diag}(S) \phi_{k}^{*}(S, V)=-\frac{1}{2 A_{k+1}}\left(\mathbf{E}_{k}\left[Y_{k} Y_{k}^{T}\right]\right)^{-1}\left(\mathbf{E}_{k}\left[C_{k+1}\left(\operatorname{diag}\left(R_{k}\right) S\right) Y_{k}\right]\right. \\
\left.+2 V e^{r \Delta t_{k}} \mathbf{E}_{k}\left[Y_{k}\right]\right) .
\end{gathered}
$$

Using (4.12) we write (4.10) as

$$
\begin{aligned}
J_{k}(S, V)=\inf _{\phi_{k}} & \left\{A _ { k + 1 } \left(\phi_{k}^{T} \operatorname{diag}(S) \mathbf{E}_{k}\left[Y_{k} Y_{k}^{T}\right] \operatorname{diag}(S) \phi_{k}+V^{2} e^{2 r \Delta t_{k}}\right.\right. \\
& \left.+2 V e^{r \Delta t_{k}} \phi_{k}^{T} \operatorname{diag}(S) \mathbf{E}_{k}\left[Y_{k}\right]\right) \\
& +\mathbf{E}_{k}\left[C_{k+1}(\operatorname{diag}(R) S)\right] V e^{r \Delta t_{k}} \\
& +\phi_{k}^{T} \operatorname{diag}(S) \mathbf{E}_{k}\left[C_{k+1}\left(\operatorname{diag}\left(R_{k}\right) S\right) Y_{k}\right] \\
& \left.+\mathbf{E}_{k}\left[D_{k+1}\left(\operatorname{diag}\left(R_{k}\right) S\right)\right]\right\}
\end{aligned}
$$

Substituting (4.15) into (4.16) we find the expression for $J_{k}(S, V)$ :

$$
\begin{aligned}
J_{k} & (S, V)=A_{k+1} V^{2} e^{2 r \Delta t_{k}}\left(1-\mathbf{E}_{k}\left[Y_{k}^{T}\right]\left(\mathbf{E}_{k}\left[Y_{k} Y_{k}^{T}\right]\right)^{-1} \mathbf{E}_{k}\left[Y_{k}\right]\right) \\
+ & V e^{r \Delta t_{k}} \mathbf{E}_{k}\left[C_{k+1}\left(\operatorname{diag}\left(R_{k}\right) S\right)\left(1-Y_{k}^{T}\left(\mathbf{E}_{k}\left[Y_{k} Y_{k}^{T}\right]\right)^{-1} \mathbf{E}_{k}\left[Y_{k}\right]\right)\right] \\
+ & \mathbf{E}_{k}\left[D_{k+1}\left(\operatorname{diag}\left(R_{k}\right) S\right)\right] \\
- & \frac{1}{4 A_{k+1}} \mathbf{E}_{k}\left[C_{k+1}\left(\operatorname{diag}\left(R_{k}\right) S\right) Y_{k}^{T}\right]\left(\mathbf{E}_{k}\left[Y_{k} Y_{k}^{T}\right]\right)^{-1} \times \\
\quad & \times \mathbf{E}_{k}\left[C_{k+1}\left(\operatorname{diag}\left(R_{k}\right) S\right) Y_{k}\right]
\end{aligned}
$$


And therefore we have

$$
J_{k}(S, V)=A_{k} V^{2}+C_{k}(S) V+D_{k}(S)
$$

with

$$
\begin{aligned}
A_{k} & =A_{k+1} e^{2 r \Delta t_{k}}\left(1-\mathbf{E}_{k}\left[Y_{k}^{T}\right]\left(\mathbf{E}_{k}\left[Y_{k} Y_{k}^{T}\right]\right)^{-1} \mathbf{E}_{k}\left[Y_{k}\right]\right) \\
C_{k}(S) & =e^{r \Delta t_{k}} \mathbf{E}_{k}\left[C_{k+1}\left(\operatorname{diag}\left(R_{k}\right) S\right)\left(1-Y_{k}^{T}\left(\mathbf{E}_{k}\left[Y_{k} Y_{k}^{T}\right]\right)^{-1} \mathbf{E}_{k}\left[Y_{k}\right]\right)\right] \\
D_{k} & =-\frac{1}{4 A_{k+1}} \mathbf{E}_{k}\left[C_{k+1}\left(\operatorname{diag}\left(R_{k}\right) S\right) Y_{k}^{T}\right]\left(\mathbf{E}_{k}\left[Y_{k} Y_{k}^{T}\right]\right)^{-1} \mathbf{E}_{k}\left[C_{k+1}\left(\operatorname{diag}\left(R_{k}\right) S\right) Y_{k}\right] \\
& +\mathbf{E}_{k}\left[D_{k+1}\left(\operatorname{diag}\left(R_{k}\right) S\right)\right] .
\end{aligned}
$$

The only thing left to prove is that $A_{k}>0$. Consider random vector

$$
Y_{k}^{T}\left(\mathbf{E}_{k}\left[Y_{k} Y_{k}^{T}\right]\right)^{-1} \mathbf{E}_{k}\left[Y_{k}\right] .
$$

Note that $\mathbb{P}\left(Y_{k}^{T}\left(\mathbf{E}_{k}\left[Y_{k} Y_{k}^{T}\right]\right)^{-1} \mathbf{E}_{k}\left[Y_{k}\right] \neq 1\right)>0$. Otherwise the coordinates of $Y_{k}$ would be linearly dependent, which contradicts the model assumptions. Then the following inequality holds

$$
0<\mathbf{E}_{k}\left[\left(1-Y_{k}^{T}\left(\mathbf{E}_{k}\left[Y_{k} Y_{k}^{T}\right]\right)^{-1} \mathbf{E}_{k}\left[Y_{k}\right]\right)^{2}\right]
$$

If we denote

$$
N_{k}=\left(\mathbf{E}_{k}\left[Y_{k} Y_{k}^{T}\right]\right)^{-1},
$$

then rewriting the right part of (4.18) we get

$$
\begin{aligned}
0 & <\mathbf{E}_{k}\left[1+\mathbf{E}_{k}\left[Y_{k}^{T}\right] N_{k} Y_{k} Y_{k}^{T} N_{k} \mathbf{E}_{k}[Y]-2 Y_{k}^{T} N_{k} \mathbf{E}_{k}\left[Y_{k}\right]\right] \\
& =1+\mathbf{E}_{k}\left[Y_{k}^{T}\right] N_{k} \mathbf{E}_{k}\left[Y_{k}\right]-2 \mathbf{E}_{k}\left[Y_{k}^{T}\right] N_{k} \mathbf{E}_{k}\left[Y_{k}\right] \\
& =1-\mathbf{E}_{k}\left[Y_{k}^{T}\right] N_{k} \mathbf{E}_{k}\left[Y_{k}\right] .
\end{aligned}
$$

Therefore

$$
1-\mathbf{E}_{k}\left[Y_{k}^{T}\right]\left(\mathbf{E}_{k}\left[Y_{k} Y_{k}^{T}\right]\right)^{-1} \mathbf{E}_{k}\left[Y_{k}\right]>0 .
$$

And since by the induction assumption $A_{k+1}>0$ we conclude that $A_{k}>0$.

Proposition 4.3.1 gives us an explicit recipe for our optimization procedure. Start at $t_{N}$ with

$$
\begin{aligned}
A_{N} & =1 \\
C_{N}(S) & =-2 \Phi(S) \\
D_{N}(S) & =\Phi^{2}(S)
\end{aligned}
$$

and then step by step calculate optimal weights $\phi_{k}^{*}$ using (4.15) and coefficients $A_{k}, C_{k}(S)$ and $D_{k}(S)$ using equations (4.17). 


\subsection{The Optimal Initial Capital}

In section 4.3 we have solved the minimization problem simultaneously for all values of the initial wealth. This section is focused on the properties of the optimal initial capital e.g. $V_{0}^{*}$ solving

$$
J_{0}\left(S, V_{0}^{*}(S)\right)=\min _{V} J_{0}(S, V) .
$$

First we show that under the assumption of independent increments the density of the variance-optimal measure (see definition 3.2.2) can be presented as a product certain functions, which can be interpreted as one-step densities.

As a straightforward corollary of proposition 4.3.1 we get an explicit form for the optimal initial capital.

Proposition 4.4.1 The solution of the problem (4.21) is given by

$$
V_{0}^{*}=-\frac{C_{0}(S)}{2 A_{0}}
$$

with $A_{0}$ and $C_{0}(S)$ defined in (4.20) and (4.17).

Definition 4.4.1 For each $N$ we define a signed measure $\mathbb{Q}_{N}$ by specifying its Radon-Nikodym derivative with respect to the real-world measure $\mathbb{P}$ :

$$
\begin{aligned}
\frac{d \mathbb{Q}_{N}}{d \mathbb{P}} & :=m_{t_{1} \mid t_{0}} m_{t_{2} \mid t_{1}} \ldots m_{t_{N} \mid t_{N-1}} \\
m_{t_{k+1} \mid t_{k}} & =\frac{1-Y_{k}^{T}\left(\boldsymbol{E}_{k}\left[Y_{k} Y_{k}^{T}\right]\right)^{-1} \boldsymbol{E}_{k}\left[Y_{k}\right]}{1-\boldsymbol{E}_{k}\left[Y_{k}^{T}\right]\left(\boldsymbol{E}_{k}\left[Y_{k} Y_{k}^{T}\right]\right)^{-1} \boldsymbol{E}_{k}\left[Y_{k}\right]},
\end{aligned}
$$

(by (4.19) the denominator is not equal to 0.)

Measure $\mathbb{Q}^{N}$ is defined in [47] where it is used for the computation of the optimal initial capital. Our setting allows us to compute $V_{0}^{*}$ directly once $C_{0}(S)$ is known. Nevertheless below we present the proofs that $\mathbb{Q}^{N}$ is the variance-optimal measure of Schweizer [45] for the case of independent returns and that $V_{0}^{*}$ defined in (4.21) is equal to the expectation of the payoff $\Phi$ with respect to the measure $\mathbb{Q}^{N}$. In the following section we prove that the density of $\mathbb{Q}^{N}$ converges to the density of the risk-neutral measure in $L^{2}$ as the distance between hedging dates goes to 0 .

Proposition 4.4.2 Under the assumption of independent returns the measure $\mathbb{Q}_{N}$ is the m-dimensional version of the variance-optimal measure of Schweizer [45] (see definition 3.2.2 of Chapter 3). 
Proof By a backward induction in $k$ we prove that for all $k=1, \ldots, N$ the following two equalities hold:

$$
\frac{1-\beta_{k} \Delta S_{k-1}}{\mathbf{E}\left[1-\beta_{k} \Delta S_{k-1}\right]}=m_{t_{k} \mid t_{k-1}}
$$

and

$$
\frac{\mathbf{E}_{k-1}\left[1-\beta_{k} \Delta S_{k-1}\right]}{\mathbf{E}_{k-1}\left[1-\beta_{k} \Delta S_{k-1}\right]^{2}}=1
$$

$1 . k=N$.

$$
\beta_{N}=\frac{\mathbf{E}_{N-1}\left[\Delta S_{N-1}\right]}{\mathbf{E}_{N-1}\left[\Delta S_{N-1}^{2}\right]}=\frac{\mathbf{E}_{N-1}\left[R_{N-1}-1\right]}{S_{N-1} \mathbf{E}_{N-1}\left[R_{N-1}-1\right]^{2}}
$$

Remember that Schweizer assumes discounted asset prices, so

$$
R_{k}-1=Y_{k}, \quad \text { for all } k=0, \ldots, N-1 \text {. }
$$

and

$$
\beta_{N}=\frac{\mathbf{E}_{N-1}\left[Y_{N-1}\right]}{S_{N-1} \mathbf{E}_{N-1}\left[Y_{N-1}\right]^{2}}
$$

Then

$$
1-\beta_{N} \Delta S_{N-1}=1-\frac{Y_{N-1} \mathbf{E}_{N-1}\left[Y_{N-1}\right]}{\mathbf{E}_{N-1}\left[Y_{N-1}^{2}\right]}
$$

and

$$
\frac{1-\beta_{N} \Delta S_{N-1}}{\mathbf{E}_{N-1}\left[1-\beta_{N} \Delta S_{N-1}\right]}=\frac{\mathbf{E}_{N-1}\left[Y_{N-1}^{2}\right]-Y_{N-1} \mathbf{E}_{N-1}\left[Y_{N-1}\right]}{\mathbf{E}_{N-1}\left[Y_{N-1}^{2}\right]-\left(\mathbf{E}_{N-1}\left[Y_{N-1}\right]\right)^{2}}=m_{t_{N} \mid t_{N-1}}
$$

which proves (4.23). Now consider

$$
\begin{aligned}
\frac{\mathbf{E}_{N-1}\left[1-\beta_{N} \Delta S_{N-1}\right]}{\mathbf{E}_{N-1}\left[1-\beta_{N} \Delta S_{N-1}\right]^{2}} & =\frac{1-\frac{\left(\mathbf{E}_{N-1}\left[Y_{N-1}\right]\right)^{2}}{\mathbf{E}_{N-1}\left[Y_{N-1}^{2}\right]}}{1-2 \frac{\left(\mathbf{E}_{N-1}\left[Y_{N-1}\right]\right)^{2}}{\mathbf{E}_{N-1}\left[Y_{N-1}^{2}\right]}+\frac{\mathbf{E}_{N-1}\left[Y_{N-1}^{2}\right]\left(\mathbf{E}_{N-1}\left[Y_{N-1}\right]\right)^{2}}{\left(\mathbf{E}_{N-1}\left[Y_{N-1}^{2}\right]\right)^{2}}} \\
& =\frac{\mathbf{E}_{N-1}\left[Y_{N-1}^{2}\right]\left(\mathbf{E}_{N-1}\left[Y_{N-1}^{2}\right]-\left(\mathbf{E}_{N-1}\left[Y_{N-1}\right]\right)^{2}\right)}{\mathbf{E}_{N-1}\left[Y_{N-1}^{2}\right]\left(\mathbf{E}_{N-1}\left[Y_{N-1}^{2}\right]-\left(\mathbf{E}_{N-1}\left[Y_{N-1}\right]\right)^{2}\right)} \\
& =1,
\end{aligned}
$$


which proves (4.24).

2. Assume now that we have proved (4.23) and (4.24) for $k+1, \ldots, N$. Let us prove it for $k$.

$$
\beta_{k}=\frac{\mathbf{E}_{k-1}\left[\Delta S_{k-1} \prod_{j=k+1}^{N}\left(1-\beta_{j} \Delta S_{j-1}\right)\right]}{\mathbf{E}_{k-1}\left[\Delta S_{k-1}^{2} \prod_{j=k+1}^{N}\left(1-\beta_{j} \Delta S_{j-1}\right)^{2}\right]}
$$

By the induction assumption and because returns $R_{j}, j=0, \ldots, N-1$ are independent all $\beta_{j}, j=k+1, \ldots, N$ are independent and they all are also independent of $R_{k-1}$, therefore

$$
\beta_{k}=\frac{\mathbf{E}_{k-1}\left[\Delta S_{k-1}\right] \prod_{j=k+1}^{N} \mathbf{E}_{k-1}\left[1-\beta_{j} \Delta S_{j-1}\right]}{\mathbf{E}_{k-1}\left[\Delta S_{k-1}^{2}\right] \prod_{j=k+1}^{N} \mathbf{E}_{k-1}\left[1-\beta_{j} \Delta S_{j-1}\right]^{2}} .
$$

Then by (4.24) of the induction assumption

$$
\beta_{k}=\frac{\mathbf{E}_{k-1}\left[\Delta S_{k-1}\right]}{\mathbf{E}_{k-1}\left[\Delta S_{k-1}^{2}\right]}
$$

and the rest of the proof is similar to the case $k=N$.

As a corollary of proposition 4.4.1 we directly prove the result of Schweizer [45] that the optimal initial capital is the expectation under the varianceoptimal measure.

Theorem 4.4.3 For every $S_{0} \in \mathbb{R}^{m}$ the optimal initial capital $V_{0}^{*}\left(S_{0}\right)$ is the discounted expectation of the payoff at maturity under the signed measure $\mathbb{Q}_{N}$ :

$$
V_{0}^{*}\left(S_{0}\right)=e^{-r T} \boldsymbol{E}^{\mathbb{Q}_{N}}\left[\Phi\left(S_{N}\right)\right]
$$

Proof (of Theorem 4.4.3) Let us denote

$$
\begin{aligned}
\tilde{V}_{k}(S) & :=-\frac{C_{k}(S)}{2 A_{k}} \\
m_{t_{k}} & :=m_{t_{k+1} \mid t_{k}} \ldots m_{t_{N} \mid t_{N-1}}, k=0, \ldots, N-1 \\
m_{t_{N}} & :=1
\end{aligned}
$$


and prove for all $k$ that

$$
\tilde{V}_{k}\left(S_{k}\right)=e^{-r\left(T-t_{k}\right)} \mathbf{E}_{k}^{\mathbb{P}}\left[m_{t_{k}} \Phi\left(S_{N}\right)\right]
$$

by backward induction in $k$. This will prove the statement of the theorem, because $\tilde{V}_{0}=V_{0}^{*}$.

1. $k=N$ :

$$
\tilde{V}_{N}\left(S_{N}\right)=-\frac{C_{N}\left(S_{N}\right)}{2 A_{N}}=\Phi\left(S_{N}\right)=\mathbf{E}_{N}^{\mathbb{P}}\left[m_{t_{N}} \Phi\left(S_{N}\right)\right] .
$$

2. Suppose the statement has been proved for all $i=k+1, \ldots, n$. Now we will prove it for $i=k$. Using (4.17) we get:

$$
\begin{aligned}
\tilde{V}_{k}\left(S_{k}\right) & =-\frac{C_{k}\left(S_{k}\right)}{2 A_{k}} \\
& =-e^{-r \Delta t_{k}} \mathbf{E}_{k}^{\mathbb{P}}\left[\frac{C_{k+1}\left(\operatorname{diag}\left(R_{k}\right) S_{k}\right)\left(1-Y_{k}^{T}\left(\mathbf{E}_{k}\left[Y_{k} Y_{k}^{T}\right]\right)^{-1} \mathbf{E}_{k}\left[Y_{k}\right]\right)}{2 A_{k+1}\left(1-\mathbf{E}_{k}\left[Y_{k}\right]^{T}\left(\mathbf{E}_{k}\left[Y_{k} Y_{k}^{T}\right]\right)^{-1} \mathbf{E}_{k}\left[Y_{k}\right]\right)}\right] \\
& =e^{-r \Delta t_{k}} \mathbf{E}_{k}^{\mathbb{P}}\left[\tilde{V}_{k+1}\left(\operatorname{diag}\left(R_{k}\right) S_{k}\right) m_{t_{k+1} \mid t_{k}}\right]
\end{aligned}
$$

and by the induction assumption

$$
\tilde{V}_{k+1}\left(S_{k+1}\right)=e^{-r\left(T-t_{k+1}\right)} \mathbf{E}_{k+1}^{\mathbb{P}}\left[m_{t_{k+1}} \Phi\left(S_{N}\right)\right] .
$$

So if we substitute (4.27) in (4.26) we find

$$
\begin{aligned}
\tilde{V}_{k}\left(S_{k}\right) & =e^{-r\left(T-t_{k}\right)} \mathbf{E}_{k}^{\mathbb{P}}\left[\mathbf{E}_{k+1}^{\mathbb{P}}\left[m_{t_{k+1}} \Phi\left(S_{N}\right)\right] m_{t_{k+1} \mid t_{k}}\right] \\
& =e^{-r\left(T-t_{k}\right)} \mathbf{E}_{k}^{\mathbb{P}}\left[m_{t_{k}} \Phi\left(S_{N}\right)\right] .
\end{aligned}
$$

This proves (4.25) and therefore

$$
V_{0}^{*}\left(S_{0}\right)=\tilde{V}_{0}\left(S_{0}\right)=e^{-r\left(T-t_{0}\right)} \mathbf{E}^{\mathbb{P}}\left[\frac{d \mathbb{Q}_{N}}{d \mathbb{P}} \Phi\left(S_{N}\right)\right]=e^{-r T} \mathbf{E}^{\mathbb{Q}_{N}}\left[\Phi\left(S_{N}\right)\right],
$$

which is the statement we had to prove.

\subsection{Continuous Dynamics}

In this section we show that if asset returns are independent the $L^{2}$ limit of the Radon-Nikodym derivative of the variance-optimal measure from definition 4.4.1 is the Radon-Nikodym derivative of the risk-neutral measure of 
the complete limit model and that the optimal initial capital converges to the Black-Scholes price as the number of the hedging dates goes to infinity. From now on we assume that the number of underlying assets is one.

Consider the generalized Black-Scholes model with the underlying asset price process $\tilde{S}$ and deterministic drift and volatility functions depending only on time. Note that if they also depend on the underlying asset price the returns fail to be independent. Indeed, take $R_{k}$ and $R_{k+1}$ for some $k$. Then

$$
\mathbf{E}\left[R_{k} R_{k+1}\right]=\mathbf{E}\left[\frac{S_{k+2}}{S_{k}}\right]
$$

which is not the same as

$$
\mathbf{E}\left[R_{k}\right] \mathbf{E}\left[R_{k+1}\right]=\mathbf{E}\left[\frac{S_{k+1}}{S_{k}}\right] \mathbf{E}\left[\frac{S_{k+2}}{S_{k+1}}\right]
$$

in a general model. Define

$$
\begin{aligned}
d \tilde{S}(t) & =\mu(t) \tilde{S}(t) d t+\sigma(t) \tilde{S}(t) d W(t), t \in[0, T] \\
\tilde{S}(0) & =\tilde{S}_{0} \\
d B(t) & =r B(t) d t \\
B(0) & =B_{0}
\end{aligned}
$$

We denote the filtration of the continuous model generated by the price process $\tilde{S}$ by $\left\{\tilde{\mathcal{F}}_{t}\right\}_{0 \leq t \leq T}$. We assume $r \in \mathbb{R}^{+}$to be constant for the sake of simplicity, the model might easily be extended to the case where $r$ is an arbitrary function of time. $\mu:[0, T] \rightarrow \mathbb{R}$ and $\sigma:[0, T] \rightarrow \mathbb{R}$ are given continuous functions satisfying the conditions $(2.4)-(2.5)$.

We define the discrete model for the time set $\mathcal{T}=\left\{t_{0}=0, \ldots, t_{N}=T\right\}$ as

$$
\begin{aligned}
S_{k} & =\tilde{S}\left(t_{k}\right), \quad k=0, \ldots, N \\
R_{k} & =\frac{S_{k+1}}{S_{k}}, \quad k=0, \ldots, N-1 .
\end{aligned}
$$

Note that there is an analytical solution for $\tilde{S}(t), t \in[0, T]$ :

$$
\tilde{S}(t)=\tilde{S}_{0} \exp \left\{\int_{0}^{t}\left(\mu(u)-\frac{1}{2} \sigma^{2}(u)\right) d u+\int_{0}^{t} \sigma(u) d W(u)\right\},
$$

and thus

$$
R_{k}=\exp \left\{\int_{t_{k}}^{t_{k+1}}\left(\mu(u)-\frac{1}{2} \sigma^{2}(u)\right) d u+\int_{t_{k}}^{t_{k+1}} \sigma(u) d W(u)\right\}
$$


for all $k=0, \ldots, N-1$ and therefore the variables $R_{k}$ are independent. Moreover the conditional and unconditional expectations are the same:

$$
\begin{aligned}
& \mathbf{E}\left[R_{k}\right]=\mathbf{E}_{k}\left[R_{k}\right]=\exp \left\{\int_{t_{k}}^{t_{k+1}} \mu(u) d u\right\}, \\
& \mathbf{E}\left[R_{k}^{2}\right]=\mathbf{E}_{k}\left[R_{k}^{2}\right]=\exp \left\{\int_{t_{k}}^{t_{k+1}}\left(2 \mu(u)+\sigma^{2}(u)\right) d u\right\} .
\end{aligned}
$$

In the rest of this section we are going to prove that the Radon-Nikodym derivatives of the variance-optimal measures $\mathbb{Q}^{N}$ of the discrete model (4.30)(4.31) with respect to the real-world measure $\mathbb{P}$ converge in $L^{2}$ to the RadonNikodym derivative of the risk-neutral measure $\mathbb{Q}$ of the continuous model (4.28)-(4.29) with respect to the real-world measure and subsequently that the optimal initial capital $V_{0}^{*}(S)$ converges to the no-arbitrage price $F(0, S)$ in the model (4.28)-(4.29) as the distance between the hedging dates goes to 0 .

Theorem 4.5.1 Assume the generalized Black-Scholes model (4.28)-(4.29) with constant interest rate $r$. Suppose $\mu(\cdot)$ and $\sigma(\cdot)$ are continuous on $[0, T]$, $\mu(\cdot)$ is continuously differentiable on $[0, T]$ and $\sigma(t)>c$ for some $c>0$ and for all $t \in[0, T]$. Then the Radon-Nikodym derivative of the variance-optimal measure of the discrete model (4.30)-(4.31) with respect to the real-world one converges in $L^{2}$ to the Radon-Nikodym derivative of the risk-neutral measure in model (4.28)-(4.29) as the maximal distance between hedging dates goes to 0:

$$
\frac{d \mathbb{Q}^{N}}{d \mathbb{P}} \stackrel{L^{2}}{\rightarrow} \frac{d \mathbb{Q}}{d \mathbb{P}}, \quad \max _{k=0, \ldots, N-1} \Delta t_{k} \rightarrow 0
$$

Proof We have to prove that

$$
\prod_{k=0}^{N-1} m_{t_{k+1} \mid t_{k}}^{N} \stackrel{L^{2}}{\rightarrow} \exp \left\{-\int_{0}^{T} \lambda(u) d W(u)-\frac{1}{2} \int_{0}^{T} \lambda^{2}(u) d u\right\}, \max _{k=0, \ldots, N-1} \Delta t_{k} \rightarrow 0
$$

where $m_{t_{k+1 \mid t_{k}}}^{N}$ is the one-dimensional version of (4.22):

$$
\begin{aligned}
m_{t_{k+1 \mid t_{k}}}^{N} & =\frac{1-Y_{k} \mathbf{E}_{k}\left[Y_{k}\right] / \mathbf{E}_{k}\left[Y_{k}^{2}\right]}{1-\left(\mathbf{E}_{k}\left[Y_{k}\right]\right)^{2} / \mathbf{E}_{k}\left[Y_{k}^{2}\right]}=\frac{\mathbf{E}_{k}\left[Y_{k}^{2}\right]-Y_{k} \mathbf{E}_{k}\left[Y_{k}\right]}{\mathbf{E}_{k}\left[Y_{k}^{2}\right]-\left(\mathbf{E}_{k}\left[Y_{k}\right]\right)^{2}}, \\
Y_{k} & =R_{k}-e^{r \Delta t_{k}},
\end{aligned}
$$


$k=0, \ldots, N-1$, and where $\lambda(t)$ is the market price of risk in the continuous model (4.28)-(4.29):

$$
\lambda(t)=\frac{\mu(t)-r}{\sigma(t)}, \quad t \in[0, T]
$$

In other words we have to prove that

$$
\left\|\prod_{k=0}^{N-1} m_{t_{k+1 \mid t_{k}}}^{N}-\exp \left\{-\int_{0}^{T} \lambda(u) d W(u)-\frac{1}{2} \int_{0}^{T} \lambda^{2}(u) d u\right\}\right\|_{L^{2}} \rightarrow 0
$$

if $\max _{k=0, \ldots, N-1} \Delta t_{k} \rightarrow 0$, which is equivalent to

$$
\mathbf{E}\left[\left(\prod_{k=0}^{N-1} m_{t_{k+1 \mid t_{k}}}^{N}-\exp \left\{-\int_{0}^{T} \lambda(u) d W(u)-\frac{1}{2} \int_{0}^{T} \lambda^{2}(u) d u\right\}\right)^{2}\right] \rightarrow 0,
$$

if $\max _{k=0, \ldots, N-1} \Delta t_{k} \rightarrow 0$.

Let us fix $N$ and rewrite the expression for this expectation.

$$
\begin{aligned}
& \mathbf{E}\left[\left(\prod_{k=0}^{N-1} m_{t_{k+1 \mid t_{k}}}^{N}-\exp \left\{-\int_{0}^{T} \lambda(u) d W(u)-\frac{1}{2} \int_{0}^{T} \lambda^{2}(u) d u\right\}\right)^{2}\right] \\
& =\mathbf{E}\left[\left(\prod_{k=0}^{N-1} m_{t_{k+1 \mid t_{k}}}^{N}\right)^{2}\right]+\mathbf{E}\left[\exp \left\{-2 \int_{0}^{T} \lambda(u) d W(u)-\int_{0}^{T} \lambda^{2}(u) d u\right\}\right] \\
& -2 \mathbf{E}\left[\prod_{k=0}^{N-1} m_{t_{k+1 \mid t_{k}}^{N}} \exp \left\{-\int_{0}^{T} \lambda(u) d W(u)-\frac{1}{2} \int_{0}^{T} \lambda^{2}(u) d u\right\}\right] \\
& =\mathbf{E}\left[\left(\frac{d \mathbb{Q}^{N}}{d \mathbb{P}}\right)^{2}\right]-2 \mathbf{E}\left[\frac{d \mathbb{Q}^{N}}{d \mathbb{P}} \frac{d \mathbb{Q}}{d \mathbb{P}}\right]+\mathbf{E}\left[\left(\frac{d \mathbb{Q}}{d \mathbb{P}}\right)^{2}\right] .
\end{aligned}
$$

We calculate each of the terms separately. The first one is

$\mathbf{E}\left[\left(\frac{d \mathbb{Q}^{N}}{d \mathbb{P}}\right)^{2}\right]=\mathbf{E}\left[\left(\prod_{k=0}^{N-1} m_{t_{k+1 \mid t_{k}}}^{N}\right)^{2}\right]=\mathbf{E}\left[\prod_{k=0}^{N-1}\left(\frac{\mathbf{E}_{k}\left[Y_{k}^{2}\right]-Y_{k} \mathbf{E}_{k}\left[Y_{k}\right]}{\mathbf{E}_{k}\left[Y_{k}^{2}\right]-\left(\mathbf{E}_{k}\left[Y_{k}\right]\right)^{2}}\right)^{2}\right]$.

Since all $R_{k}, k=0, \ldots, N-1$ are independent, $Y_{k}, k=0, \ldots, N-1$ are also independent and so are $\mathbf{E}_{k}\left[Y_{k}\right], k=0, \ldots, N-1$ and therefore all $m_{t_{k+1 \mid t_{k}}}^{N}$, 
$k=0, \ldots, N-1$ are independent and the expectation of the product is thus the product of expectations:

$$
\mathbf{E}\left[\left(\frac{d \mathbb{Q}^{N}}{d \mathbb{P}}\right)^{2}\right]=\prod_{k=0}^{N-1} \mathbf{E}\left[\left(\frac{\mathbf{E}_{k}\left[Y_{k}^{2}\right]-Y_{k} \mathbf{E}_{k}\left[Y_{k}\right]}{\mathbf{E}_{k}\left[Y_{k}^{2}\right]-\left(\mathbf{E}_{k}\left[Y_{k}\right]\right)^{2}}\right)^{2}\right]
$$

By (4.33) and (4.34) we write that

$$
\begin{gathered}
\mathbf{E}_{k}\left[Y_{k}\right]=\mathbf{E}\left[Y_{k}\right]=\exp \left\{\int_{t_{k}}^{t_{k+1}} \mu(u) d u\right\}-e^{r \Delta t_{k}} \\
\mathbf{E}_{k}\left[Y_{k}^{2}\right]=\mathbf{E}\left[Y_{k}^{2}\right]=\exp \left\{\int_{t_{k}}^{t_{k+1}}\left(2 \mu(u)+\sigma^{2}(u)\right) d u\right\} \\
-2 e^{r \Delta t_{k}} \exp \left\{\int_{t_{k}}^{t_{k+1}} \mu(u) d u\right\}+e^{2 r \Delta t_{k}} .
\end{gathered}
$$

Then for all $k=0, \ldots, N-1$

$$
\begin{aligned}
& \mathbf{E}\left[\left(\frac{\mathbf{E}_{k}\left[Y_{k}^{2}\right]-Y_{k} \mathbf{E}_{k}\left[Y_{k}\right]}{\mathbf{E}_{k}\left[Y_{k}^{2}\right]-\left(\mathbf{E}_{k}\left[Y_{k}\right]\right)^{2}}\right)^{2}\right]=\mathbf{E}\left[\left(\frac{\mathbf{E}\left[Y_{k}^{2}\right]-Y_{k} \mathbf{E}\left[Y_{k}\right]}{\mathbf{E}\left[Y_{k}^{2}\right]-\left(\mathbf{E}\left[Y_{k}\right]\right)^{2}}\right)^{2}\right] \\
& =\frac{\mathbf{E}\left[\left(\mathbf{E}\left[Y_{k}^{2}\right]\right)^{2}-2 Y_{k} \mathbf{E}\left[Y_{k}\right] \mathbf{E}\left[Y_{k}^{2}\right]+\left(Y_{k} \mathbf{E}\left[Y_{k}\right]\right)^{2}\right]}{\left(\mathbf{E}\left[Y_{k}^{2}\right]-\left(\mathbf{E}\left[Y_{k}\right]\right)^{2}\right)^{2}} \\
& =\frac{\mathbf{E}\left[Y_{k}^{2}\right]}{\mathbf{E}\left[Y_{k}^{2}\right]-\left(\mathbf{E}\left[Y_{k}\right]\right)^{2}} \\
& =1+\frac{\left(\mathbf{E}\left[Y_{k}\right]\right)^{2}}{\mathbf{E}\left[Y_{k}^{2}\right]-\left(\mathbf{E}\left[Y_{k}\right]\right)^{2}} .
\end{aligned}
$$

Using (4.36) and (4.37) we write that for all $k=0, \ldots, N-1$

$$
1+\frac{\left(\mathbf{E}\left[Y_{k}\right]\right)^{2}}{\mathbf{E}\left[Y_{k}^{2}\right]-\left(\mathbf{E}\left[Y_{k}\right]\right)^{2}}=1+\frac{\left(e^{\int_{t_{k}}^{t_{k+1}} \mu(u) d u}-e^{r \Delta t_{k}}\right)^{2}}{e^{\int_{t_{k}}^{t_{k+1}}\left(2 \mu(u)+\sigma^{2}(u)\right) d u}-e^{\int_{t_{k}}^{t_{k+1}} 2 \mu(u) d u}}
$$


and therefore the first term is

$$
\mathbf{E}\left[\left(\frac{d \mathbb{Q}^{N}}{d \mathbb{P}}\right)^{2}\right]=\prod_{k=0}^{N-1}\left\{1+\frac{\left(e^{\int_{t_{k}}^{t_{k+1}} \mu(u) d u}-e^{r \Delta t_{k}}\right)^{2}}{e^{\int_{t_{k}}^{t_{k+1}}\left(2 \mu(u)+\sigma^{2}(u)\right) d u}-e^{\int_{t_{k}}^{t_{k+1}} 2 \mu(u) d u}}\right\} .
$$

The cross-term can be rewritten as

$\begin{aligned} \mathbf{E}\left[\frac{d \mathbb{Q}^{N}}{d \mathbb{P}} \frac{d \mathbb{Q}}{d \mathbb{P}}\right] & =\mathbf{E}\left[\prod_{k=0}^{N-1} m_{t_{k+1 \mid t_{k}}}^{N} \exp \left\{-\int_{0}^{T} \lambda(u) d W(u)-\frac{1}{2} \int_{0}^{T} \lambda^{2}(u) d u\right\}\right] \\ & =\mathbf{E}\left[\prod_{k=0}^{N-1}\left\{m_{t_{k+1 \mid t_{k}}}^{N} \exp \left\{-\int_{t_{k}}^{t_{k+1}} \lambda(u) d W(u)-\frac{1}{2} \int_{t_{k}}^{t_{k+1}} \lambda^{2}(u) d u\right\}\right\}\right] .\end{aligned}$

Since exp $\left\{-\int_{0}^{T} \lambda(u) d W(u)-\frac{1}{2} \int_{0}^{T} \lambda^{2}(u) d u\right\}$ is the Radon-Nikodym derivative of the risk-neutral measure $\mathbb{Q}$ with respect to the real-world measure $\mathbb{P}$ we may write

$$
\mathbf{E}\left[\frac{d \mathbb{Q}^{N}}{d \mathbb{P}} \frac{d \mathbb{Q}}{d \mathbb{P}}\right]=\mathbf{E}^{\mathbb{Q}}\left[\frac{d \mathbb{Q}^{N}}{d \mathbb{P}}\right]=\mathbf{E}^{\mathbb{Q}}\left[\prod_{k=0}^{N-1} m_{t_{k+1} \mid t_{k}}^{N}\right]
$$

and because variables $m_{t_{k+1 \mid t_{k}}}^{N}, k=0, \ldots, N-1$ are independent we may interchange the order of the product and the expectation:

$$
\mathbf{E}\left[\frac{d \mathbb{Q}^{N}}{d \mathbb{P}} \frac{d \mathbb{Q}}{d \mathbb{P}}\right]=\prod_{k=0}^{N-1} \mathbf{E}^{\mathbb{Q}}\left[m_{t_{k+1 \mid t_{k}}}^{N}\right] .
$$

For all $k=0, \ldots, N-1$

$$
\begin{aligned}
\mathbf{E}^{\mathbb{Q}}\left[m_{t_{k+1 \mid t_{k}}}^{N}\right] & =\mathbf{E}^{\mathbb{Q}}\left[\frac{\mathbf{E}\left[Y_{k}^{2}\right]-Y_{k} \mathbf{E}\left[Y_{k}\right]}{\mathbf{E}\left[Y_{k}^{2}\right]-\left(\mathbf{E}\left[Y_{k}\right]\right)^{2}}\right] \\
& =\frac{\mathbf{E}\left[Y_{k}^{2}\right]-\mathbf{E}^{\mathbb{Q}}\left[Y_{k}\right] \mathbf{E}\left[Y_{k}\right]}{\mathbf{E}\left[Y_{k}^{2}\right]-\left(\mathbf{E}\left[Y_{k}\right]\right)^{2}} .
\end{aligned}
$$

Under the risk-neutral measure the expected return on the underlying asset is equal to the bank return:

$$
\mathbf{E}_{k}^{\mathbb{Q}}\left[R_{k}\right]=e^{r \Delta t_{k}}, \quad k=0, \ldots, N-1,
$$


hence

$$
\mathbf{E}_{k}^{\mathbb{Q}}\left[Y_{k}\right]=0, \quad k=0, \ldots, N-1,
$$

therefore

$$
\begin{aligned}
\mathbf{E}_{k}^{\mathbb{Q}}\left[m_{t_{k+1 \mid t_{k}}}^{N}\right] & =\frac{\mathbf{E}\left[Y_{k}^{2}\right]}{\mathbf{E}\left[Y_{k}^{2}\right]-\left(\mathbf{E}\left[Y_{k}\right]\right)^{2}} \\
& =1+\frac{\left(\mathbf{E}\left[Y_{k}\right]\right)^{2}}{\mathbf{E}\left[Y_{k}^{2}\right]-\left(\mathbf{E}\left[Y_{k}\right]\right)^{2}}
\end{aligned}
$$

Thus the second term is

$$
-2 \mathbf{E}\left[\frac{d \mathbb{Q}^{N}}{d \mathbb{P}} \frac{d \mathbb{Q}}{d \mathbb{P}}\right]=-2 \prod_{k=0}^{N-1}\left\{1+\frac{\left(e^{\int_{t_{k}}^{t_{k+1}} \mu(u) d u}-e^{r \Delta t_{k}}\right)^{2}}{e^{\int_{t_{k}}^{t_{k+1}}\left(2 \mu(u)+\sigma^{2}(u)\right) d u}-e^{\int_{t_{k}}^{t_{k+1}} 2 \mu(u) d u}}\right\} .
$$

Finally, the last term

$$
\begin{aligned}
\mathbf{E}\left[\left(\frac{d \mathbb{Q}}{d \mathbb{P}}\right)^{2}\right] & =\mathbf{E}\left[\exp \left\{-2 \int_{0}^{T} \lambda(u) d W(u)-\int_{0}^{T} \lambda^{2}(u) d u\right\}\right] \\
& =\exp \left\{\int_{0}^{T} \lambda^{2}(u) d u\right\} .
\end{aligned}
$$

Taking all terms back together we find that

$$
\left\|\frac{d \mathbb{Q}^{N}}{d \mathbb{P}}-\frac{d \mathbb{Q}}{d \mathbb{P}}\right\|_{L^{2}}^{2}=e^{\int^{T} \lambda^{2}(u) d u}-\prod_{k=0}^{N-1}\left\{1+\frac{\left(e^{\int_{t_{k}}^{t_{k+1}} \mu(u) d u}-e^{r \Delta t_{k}}\right)^{2}}{e^{\int_{t_{k}}^{t_{k+1}}\left(2 \mu(u)+\sigma^{2}(u)\right) d u}-e^{\int_{t_{k}}^{t_{k+1}} 2 \mu(u) d u}}\right\} .
$$

Since $\mu$ and $\sigma$ are continuous functions we may use the mean-value theorem 
(see [19]) to state that for all $k=0, \ldots, N-1$

$$
\begin{aligned}
\int_{t_{k}}^{t_{k+1}} \mu(u) d u & =\mu\left(\xi_{k}\right) \Delta t_{k}, \\
\int_{t_{k}}^{t_{k+1}} \sigma^{2}(u) d u & =\sigma^{2}\left(\zeta_{k}\right) \Delta t_{k} .
\end{aligned}
$$

for some points $\xi_{k}, \zeta_{k} \in\left[t_{k}, t_{k+1}\right]$. Then for each $k=0, \ldots, N-1$

$$
\frac{\left(e^{\int_{t_{k}}^{t_{k+1}} \mu(u) d u}-e^{r \Delta t_{k}}\right)^{2}}{e^{\int_{t_{k}}^{t_{k+1}}\left(2 \mu(u)+\sigma^{2}(u)\right) d u}-e^{\int_{t_{k}}^{t_{k+1}} 2 \mu(u) d u}}=\frac{\left(e^{\mu\left(\xi_{k}\right) \Delta t_{k}}-e^{r \Delta t_{k}}\right)^{2}}{e^{\left(2 \mu\left(\xi_{k}\right)+\sigma^{2}\left(\zeta_{k}\right)\right) \Delta t_{k}}-e^{2 \mu\left(\xi_{k}\right) \Delta t_{k}}} .
$$

Expanding exponents around zero we write for all $k=0, \ldots, N-1$ and $\Delta t_{k} \rightarrow 0$

$$
\begin{aligned}
\frac{\left(e^{\mu\left(\xi_{k}\right) \Delta t_{k}}-e^{r \Delta t_{k}}\right)^{2}}{e^{\left(2 \mu\left(\xi_{k}\right)+\sigma^{2}\left(\zeta_{k}\right)\right) \Delta t_{k}}-e^{2 \mu\left(\xi_{k}\right) \Delta t_{k}}} & =\frac{\left(\mu\left(\xi_{k}\right) \Delta t_{k}-r \Delta t_{k}+o\left(\Delta t_{k}\right)\right)^{2}}{\sigma^{2}\left(\zeta_{k}\right) \Delta t_{k}+o\left(\Delta t_{k}\right)} \\
& =\frac{\left(\mu\left(\xi_{k}\right)-r\right)^{2}}{\sigma^{2}\left(\zeta_{k}\right)} \Delta t_{k}+o\left(\Delta t_{k}\right) .
\end{aligned}
$$

So

$$
\begin{aligned}
\prod_{k=0}^{N-1}\{1+ & \left.\frac{\left(e^{\int_{t_{k}}^{t_{k+1}} \mu(u) d u}-e^{r \Delta t_{k}}\right)^{2}}{e^{\int_{t_{k}}^{t_{k+1}}\left(2 \mu(u)+\sigma^{2}(u)\right) d u}-e^{\int_{t_{k}}^{t_{k+1}}} 2 \mu(u) d u}\right\} \\
& =\prod_{k=0}^{N-1}\left\{1+\frac{\left(\mu\left(\xi_{k}\right)-r\right)^{2}}{\sigma^{2}\left(\zeta_{k}\right)} \Delta t_{k}+o\left(\Delta t_{k}\right)\right\} \\
& =\exp \left\{\sum_{k=0}^{N-1} \ln \left(1+\frac{\left(\mu\left(\xi_{k}\right)-r\right)^{2}}{\sigma^{2}\left(\zeta_{k}\right)} \Delta t_{k}+o\left(\Delta t_{k}\right)\right)\right\} .
\end{aligned}
$$

If $\mu(t)$ is continuously differentiable, then for all $k=0, \ldots, N-1$

$$
\mu\left(\xi_{k}\right)=\mu\left(\zeta_{k}\right)+\mu^{\prime}\left(\zeta_{k}\right)\left(\xi_{k}-\zeta_{k}\right)+o\left(\left|\zeta_{k}-\xi_{k}\right|\right),
$$


so for all $k=0, \ldots, N-1$

$$
\frac{\left(\mu\left(\xi_{k}\right)-r\right)^{2}}{\sigma^{2}\left(\zeta_{k}\right)} \Delta t_{k}+o\left(\Delta t_{k}\right)=\frac{\left(\mu\left(\zeta_{k}\right)-r\right)^{2}}{\sigma^{2}\left(\zeta_{k}\right)} \Delta t_{k}+o\left(\Delta t_{k}\right) .
$$

Expanding the logarithm around zero we get

$$
\begin{aligned}
\exp \left\{\sum_{k=0}^{N-1} \ln \left(1+\frac{\left(\mu\left(\xi_{k}\right)-r\right)^{2}}{\sigma^{2}\left(\zeta_{k}\right)} \Delta t_{k}+o\left(\Delta t_{k}\right)\right)\right\} \\
=\exp \left\{\sum_{k=0}^{N-1}\left(\frac{\left(\mu\left(\zeta_{k}\right)-r\right)^{2}}{\sigma^{2}\left(\zeta_{k}\right)} \Delta t_{k}+o\left(\Delta t_{k}\right)\right)\right\} \\
=\exp \left\{\sum_{k=0}^{N-1}\left(\lambda^{2}\left(\zeta_{k}\right) \Delta t_{k}+o\left(\Delta t_{k}\right)\right)\right\} \rightarrow \exp \left\{\int_{0}^{T} \lambda^{2}(u) d u\right\},
\end{aligned}
$$

if $\max _{k=0, \ldots, N-1} \Delta t_{k} \rightarrow 0$. Therefore

$$
\left\|\frac{d \mathbb{Q}^{N}}{d \mathbb{P}}-\frac{d \mathbb{Q}}{d \mathbb{P}}\right\|_{L^{2}} \rightarrow 0, \max _{k=0, \ldots, N-1} \Delta t_{k} \rightarrow 0
$$

Corollary 4.5.2 Under the assumptions of the theorem 4.5.1 and if $\Phi$ is bounded then the optimal initial capital $V_{0}^{*}$ defined in (4.21) converges to the no-arbitrage price $V$ of the contingent claim $\Phi(\tilde{S}(T))$ in the generalized Black-Scholes model (4.28)-(4.29) as the time between hedges goes to zero.

Proof By theorem 4.4.3 the optimal initial capital is the expectation of the final payoff under the variance optimal measure $\mathbb{Q}^{N}$ :

$$
\left(V_{0}^{*}\right)^{N}=\mathbf{E}^{\mathbb{Q}^{N}}\left[\Phi\left(S_{T}\right)\right]
$$

By theorem 4.5.1 the Radon-Nikodym derivatives $\frac{d \mathbb{Q}^{N}}{d \mathbb{P}}$ converge to the RadonNikodym derivative $\frac{d \mathbb{Q}}{d \mathbb{P}}$ in $L_{2}$ if $N$ goes to infinity. Therefore

$$
\frac{d \mathbb{Q}^{N}}{d \mathbb{P}} \rightarrow \frac{d \mathbb{Q}}{d \mathbb{P}}, N \rightarrow \infty \text { in distribution, }
$$

SO

$$
\left(V_{0}^{*}\right)^{N}=\mathbf{E}^{\mathbb{Q}^{N}}\left[\Phi\left(S_{T}\right)\right] \rightarrow \mathbf{E}^{\mathbb{Q}}\left[\Phi\left(S_{T}\right)\right]=V, N \rightarrow \infty .
$$




\section{Chapter 5}

\section{Model with Cost of Risk}

In this chapter we present a new approach to the optimal hedging problem. Our model maximizes the expected profit of the trader while avoiding the use of utility functions. In a standard model (e.g. such as defined in chapter 4) the maximization of the expected portfolio value would lead to unbounded strategies: since the expected return on the risky asset is bigger than the risk-free rate, borrowing more money from the bank account $B$ and investing it in a larger number of assets $S$ will result in a higher positive expected profit. Thus the optimal strategy is buying an infinite number of stocks, which results in an infinite expected profit. It also results in an infinite risk though, which the strategy does not take care of. A utility function is one of the ways to take the risk into account. As mentioned for example in [11], despite thorough research made in the field of utility maximization and the fact that it is well-developed from a theoretical point of view, the utility approach is rarely used in practice mostly due to the difficulty of defining the utility function of a trader. Therefore we choose a different way. In our model the risk is managed by means of an extra bank account with an interest smaller than the risk-free interest rate, which should be thought of as a capital reserve.

Capital reserve is the amount of money a bank has to put aside to cover an eventual loss. This amount is regulated by the Basel Committee on Banking Supervision and its Basel Capital Accords. The first of the Basel Accords, also known as Basel I was mainly focused on the credit risk. According to it the banks were required to reserve $8 \%$ of their risk-weighted assets. The full version of Basel I can be found in [3]. Basel II, the second Basel Capital Accord, (see [4] for the full version) considers not only the credit risk but also operational and market risks. The latter - the risk we are interested in for our research - is supposed to be valuated using VaR - the value at risk technique. VaR is the maximum amount at risk to be lost over a certain 
period on a certain confidence level. For more details we refer the reader to [27]. Although this technique is widely applied, it has been criticized for its strong dependence on the estimation of small probabilities [38] and weak perfomance in the event of market crashes [26].

Modeling the capital reserve as an extra bank account with an interest rate smaller than the risk-free can be seen as a slightly stylized reflection of what is happening in the real life. In general, every bank (or a trading company) has a certain profit target. At the same time, it has to remain solvent and thus the amount of capital it can put at risk is limited. This defines a target rate of return for the bank's investments. When a trader takes on a risky position, he ties up some of the bank's capital: the bank now has to put some money aside in case the trader makes a loss and the bank has to pay to the counterparty. Since this money has to be readily available, it is kept in a bank account or in some very liquid instruments with yields that can not possibly match the bank's target rate of return. So from the bank's point of view this is not a profitable enough investment. For trader's risk to be worth it, the trader has to compensate all the "lost" return associated with the solvency reserve that has to be kept by the bank in order to mitigate possible losses of the trader. Hence, from the trader's point of view every euro worth of risk is generating an interest smaller than the risk-free rate.

The extra bank account of our model is not an extra traded asset in the market, because at any time it has to contain a fixed amount of money depending on the trader's portfolio risk. The mechanism of the risk management is simple. The bigger the risk of the portfolio, the more the trader should keep in the reserve bank account, the more he or she loses because of the lower interest rate.

We assume a finite discrete time set and a binomial model for the underlying asset price process. The discrete time of the model reflects the non-continuous nature of the trading in the real life. The simplicity of the binomial structure is also a desirable feature for the traders. Nevertheless we see the continuous-time limit as a possible extension. The risk-free rate is assumed to be constant and so is the interest rate on the reserve bank account. We only consider portfolios of European options here. We model the risk of the portfolio by a function which depends on the market state and the weights of the portfolio, and we maximize the expected final value of the portfolio by optimizing the number of underlying assets with the dynamic programming technique.

Despite the absence of utility functions we may then introduce an indifference price as the amount of money, which makes the optimally behaving trader indifferent between having a certain option in his or her portfolio or not 
having it. We introduce relative prices with respect to an existing portfolio: we define buying and selling indifference prices that depend on the current trader's portfolio. Assuming a special form of the risk function based on the portfolio Greeks - the derivatives of the Black-Scholes price of the portfolio with respect to its parameters - we are able to get analytical solutions for the indifference prices. These indifference prices show a difference between bid and ask prices, they depend on the trader's existing portfolio and produce volatility smiles, which may be fitted to the market by changing the parameters of the risk function.

\subsection{The Model}

In this section we define the market model and the set of admissible trading strategies. We consider the discrete time model (4.1) with time set $\mathcal{T}=\left\{0=t_{0}<\ldots<t_{N}=T\right\}$ that we defined in chapter 4 with an additional riskless asset $Z$ :

$$
\begin{aligned}
S_{k+1} & =S_{k} R_{k}, \quad k=0, \ldots, N-1, \\
B_{k} & =e^{r t_{k}}, \quad k=0, \ldots, N, \\
Z_{k} & =e^{\tilde{r} t_{k}}, \quad k=0, \ldots, N, \\
0 & \leq \tilde{r}<r .
\end{aligned}
$$

with $S_{0}>0, R: \Omega \times \mathcal{T} \rightarrow \mathbb{R}$ and $\mathcal{F}_{k}=\sigma\left(R_{0}, \ldots, R_{k-1}\right)$. The third asset $Z$ is not traded, and therefore, the obvious arbitrage opportunity of borrowing from $Z$ and investing in $B$ is not possible.

We assume binomial dynamics for the underlying asset $S$, which is described by a special form of random variables $R_{k}$ :

$$
R_{k}=\left\{\begin{array}{ll}
u_{k} & \text { with probability } p_{k} \\
d_{k} & \text { with probability } 1-p_{k}
\end{array},\right.
$$

where

$$
\begin{aligned}
& u_{k}=\exp \left\{\left(\mu-\frac{1}{2} \sigma^{2}\right) \Delta t_{k}+\sigma \sqrt{\Delta t_{k}}\right\} \\
& d_{k}=\exp \left\{\left(\mu-\frac{1}{2} \sigma^{2}\right) \Delta t_{k}-\sigma \sqrt{\Delta t_{k}}\right\} \\
& p_{k}=\frac{1}{2}
\end{aligned}
$$

for all $k=0, \ldots, N-1$ and $\mu>r$. 
The trader holds a portfolio of $n$ European options with maturities $T_{i} \in$ $\mathcal{T} \backslash\left\{t_{0}\right\}, i=1, \ldots, n$ and payoffs $\Phi^{i}: \mathbb{R}^{+} \rightarrow \mathbb{R}, i=1, \ldots, n$. Their total payoff is a function $\Phi: \mathcal{T} \times \mathbb{R}^{+} \rightarrow \mathbb{R}$

$$
\Phi(t, S)=\sum_{i=1}^{n} \Phi^{i}(S) \delta_{T_{i}}(t)
$$

where

$$
\delta_{T_{i}}(t)=\left\{\begin{array}{lr}
1, & t=T_{i} \\
0, & \text { otherwise }
\end{array}\right.
$$

we denote the payoff at time $t_{k}$ as $\Phi_{k}$ (unlike the number of an option, which is denoted by a superscript, the time point is denoted by a subscript):

$$
\Phi_{k}(S)=\Phi\left(t_{k}, S\right), \quad k=1, \ldots, N
$$

The trading strategies are adapted vector-valued stochastic processes $\pi=\left(h^{S}, h^{B}, h^{Z}\right)$ with $\pi: \Omega \times \mathcal{T} \rightarrow \mathbb{R}^{3}$, where $h^{S}$ is the number of stocks, $h^{B}$ is the number of bonds in the bank account $B$ and $h^{Z}$ is the number of bonds in the capital reserve account $Z$. All option payoffs received or paid are invested in or withdrawn from the bank account $B$. In other words at time $t_{i}$ we buy $\frac{\Phi_{i}\left(S_{i}\right)}{B_{i}}$ bonds for the price $B_{i}$ when $\Phi_{i}\left(S_{i}\right)>0$ or sell them when $\Phi_{i}\left(S_{i}\right)<0$. At time $t_{k}>t_{i}$ this investment is worth $\Phi_{i}\left(S_{i}\right) \frac{B_{k}}{B_{i}}$. If we denote the value process of the portfolio by $V$, then the value of the hedging portfolio at time $t_{k}$ is

$$
V_{k}^{\pi}=h_{k}^{S} S_{k}+h_{k}^{B} B_{k}+h_{k}^{Z} Z_{k}+\sum_{i=1}^{k} \Phi_{i}\left(S_{i}\right) \frac{B_{k}}{B_{i}} .
$$

Definition 5.1.1 The set of admitted trading strategies $\mathcal{A}$ consists of all adapted strategies $\pi$ satisfying two conditions:

1. $\pi$ is self-financing when the cash inflow from the expiring options' payoffs are included:

$$
\begin{gathered}
\Delta V_{k}^{\pi}=h_{k}^{S} \Delta S_{k}+h_{k}^{B} \Delta B_{k}+h_{k}^{Z} \Delta Z_{k}+\sum_{i=1}^{k} \Phi_{i}\left(S_{i}\right) \frac{\Delta B_{k}}{B_{i}} \\
+\Phi_{k+1}\left(S_{k+1}\right) .
\end{gathered}
$$

2. The size of the prescribed capital reserve is a fixed function of the portfolio weights and the market prices:

$$
h_{k}^{Z}=f_{k}\left(h_{k}^{S}, h_{k}^{B}, S_{k}, B_{k}, Z_{k}\right) .
$$

The function $f_{k}$ is called the risk function. 
The goal of the trader is to maximize the expectation of his or her final position over all admitted strategies, for given initial wealth $V_{0}$ :

$$
\max _{\pi \in \mathcal{A}} \mathbf{E}\left[V_{T}^{\pi}\right] .
$$

In section 5.1.2 we specify functions $f_{k}$ in such a way that the dependence on various variables comes from a functional dependence on the payoff $\Phi$, so that the optimal strategy depends on the portfolio of options, which is not the case otherwise.

\subsubsection{The Optimization Problem Setup}

The state variable for our problem is the pair $(S, V) \in \mathbb{R}^{+} \times \mathbb{R}$, where $S$ is the price of the underlying asset and $V$ is the total value of the hedging portfolio. We define the reward functional as

$$
J^{\pi}=\mathbf{E}\left[V_{T}^{\pi}\right]
$$

and we maximize it over all admitted strategies:

$$
\max _{\pi \in \mathcal{A}} J^{\pi}
$$

From now on, for the sake of easier notation, we will omit the superscripts $\pi$ and write $V_{k}$ instead of $V_{k}^{\pi}$.

The dynamics of $S$ are given by the equation

$$
S_{k+1}=S_{k} R_{k}, k=0, \ldots, N-1 .
$$

The dynamics for $V$ follow from the conditions on admitted strategies (5.4) and (5.5). Consider an arbitrary $k=0, \ldots, N-1$. From the first condition we have:

$\Delta V_{k}=h_{k}^{S} S_{k} \frac{\Delta S_{k}}{S_{k}}+\left(h_{k}^{B} B_{k}+\sum_{i=1}^{k} \Phi_{i}\left(S_{i}\right) \frac{B_{k}}{B_{i}}\right) \frac{\Delta B_{k}}{B_{k}}+h_{k}^{Z} Z_{k} \frac{\Delta Z_{k}}{Z_{k}}+\Phi_{k+1}\left(S_{k} R_{k}\right)$

And from the second one, the amount of money on the reserve bank account $h_{k}^{Z}$ is determined by the risk function $f_{k}$ :

$$
\begin{aligned}
\Delta V_{k}=h_{k}^{S} & S_{k} \frac{\Delta S_{k}}{S_{k}}+\left(h_{k}^{B} B_{k}+\sum_{i=1}^{k} \Phi_{i}\left(S_{i}\right) \frac{B_{k}}{B_{i}}\right) \frac{\Delta B_{k}}{B_{k}} \\
& +f_{k}\left(h_{k}^{S}, h_{k}^{B}, S_{k}, B_{k}, Z_{k}\right) Z_{k} \frac{\Delta Z_{k}}{Z_{k}}+\Phi_{k+1}\left(S_{k} R_{k}\right)
\end{aligned}
$$


By the definition of the portfolio value (5.3)

$$
h_{k}^{B} B_{k}+\sum_{i=1}^{k} \Phi_{i}\left(S_{i}\right) \frac{B_{k}}{B_{i}}=V_{k}-h_{k}^{S} S_{k}-h_{k}^{Z} Z_{k}
$$

and

$$
\begin{aligned}
& \frac{\Delta B_{k}}{B_{k}}=e^{r \Delta t_{k}}-1, \\
& \frac{\Delta S_{k}}{S_{k}}=R_{k}-1,
\end{aligned}
$$

So

$$
V_{k+1}=V_{k} e^{r \Delta t_{k}}+h_{k}^{S} S_{k}\left(R_{k}-e^{r \Delta t_{k}}\right)+f_{k}\left(h_{k}^{S}, h_{k}^{B}, S_{k}, B_{k}, Z_{k}\right) E_{k}+\Phi_{k+1}\left(S_{k} R_{k}\right),
$$

where

$$
E_{k}=e^{\tilde{r} t_{k}}\left(e^{\tilde{r} \Delta t_{k}}-e^{r \Delta t_{k}}\right) \leq 0, k=0, \ldots, N-1 .
$$

Since $B_{k}$ and $Z_{k}$ are deterministic and $h_{k}^{B}$ follows from $V_{k}$ and the selffinancing condition (5.4) we may write

$$
f_{k}\left(h_{k}^{S}, h_{k}^{B}, S_{k}, B_{k}, Z_{k}\right)=\rho_{k}\left(S_{k}, V_{k}, h_{k}^{S}\right) .
$$

Note that for any adapted process $h^{S}$ there exist (by (5.5) and (5.6)) unique adapted processes $h^{B}$ and $h^{Z}$ such that $\left(h^{S}, h^{B}, h^{Z}\right)$ is an admitted strategy. Therefore the optimization problem may be written as

$$
\begin{aligned}
& \max _{h^{S} \text {-adapted }} \mathbf{E}\left[V_{N}\right], \\
& \left(S_{k+1}, V_{k+1}\right)=\left(S_{k} R_{k}, V_{k} e^{r \Delta t_{k}}+h_{k}^{S} S_{k}\left(R_{k}-e^{r \Delta t_{k}}\right)+\rho_{k}\left(S_{k}, V_{k}, h_{k}^{S}\right) E_{k}\right. \\
& \left.+\Phi_{k+1}\left(S_{k} R_{k}\right)\right)
\end{aligned}
$$

So unlike Basak and Shapiro [2] case our optimization problem is unconstrained as the risk management term is embedded in the dynamics of the portfolio value.

Following the line of utility function theory as in Chapter 3 we introduce the notion of indifference prices:

Definition 5.1.2 Let $J^{X}$ be the solution to the problem (5.7) for the option portfolio $X$, then the number $p^{b}$

$$
p^{b}=e^{-r T}\left(J^{\Phi+\Psi}-J^{\Psi}\right)
$$


is called the indifference buyer's price of portfolio $\Phi$ in the presence of portfolio $\Psi$, the number $p^{s}$

$$
p^{s}=e^{-r T}\left(J^{\Psi}-J^{\Psi-\Phi}\right)
$$

is called the indifference seller's price of portfolio $\Phi$ in the presence of portfolio $\Psi$ and the number $p_{m}$

$$
p^{m}=\frac{p^{s}+p^{b}}{2}
$$

is called the indifference mid price of portfolio $\Phi$ in the presence of portfolio $\Psi$. We will use the following notations for these prices:

$$
\begin{aligned}
& \{\Phi\}_{\{\Psi\}}^{b}=p^{b}, \\
& \{\Phi\}_{\{\Psi\}}^{s}=p^{s}, \\
& \{\Phi\}_{\{\Psi\}}^{m}=p^{m} .
\end{aligned}
$$

In other words the indifference price of $\Phi$ in presence of $\Psi$ is the price that an optimally hedging trader who currently holds $\Psi$ will quote for selling or buying the portfolio $\Phi$. For example if a trader is asked to sell a portfolio $\Phi$ he or she will compare the expected profit he or she may get from his or her current position $\Psi$, and that is $J^{\Psi}$, with the expected profit he or she may get from the same position but without $\Phi$, and that is $J^{\Psi-\Phi}$, and will ask for the price which makes him or her indifferent between those two, which is the discounted difference:

$$
e^{-r T}\left(J^{\Psi}-J^{\Psi-\Phi}\right)
$$

If prices are defined in this way they do not only show a difference between the bid and the ask prices but also depend on the trader's book and allow the trader to quote competitive prices.

Here we defined the indifference prices at time zero. We may also define selling, buying and mid indifference prices $\{\Phi\}_{\{\Psi\}, k}^{s},\{\Phi\}_{\{\Psi\}, k}^{b},\{\Phi\}_{\{\Psi\}, k}^{m}$ at any time $0<t_{k} \leq T$ in the similar way, if $J^{\Phi, k}$ is the solution to the problem (5.7) for option portfolio $\Phi$ and time set $\mathcal{T}_{k}=\left\{t_{k}, \ldots, t_{N}\right\}$ :

$$
\begin{aligned}
& \{\Phi\}_{\{\Psi\}, k}^{s}=e^{-r\left(T-t_{k}\right)}\left(J^{\Psi, k}-J^{\Psi-\Phi, k}\right), \\
& \{\Phi\}_{\{\Psi\}, k}^{b}=e^{-r\left(T-t_{k}\right)}\left(J^{\Phi+\Psi, k}-J^{\Psi, k}\right), \\
& \{\Phi\}_{\{\Psi\}, k}^{m}=\frac{\{\Phi\}_{\{\Psi\}, k}^{s}+\{\Phi\}_{\{\Psi\}, k}^{b}}{2} .
\end{aligned}
$$




\subsubsection{The Risk Measure}

Our choice for the risk measures $\rho_{k}$ is dictated by the following considerations. First of all we would like our risk function to be expressed in terms of observable market parameters which are immediately recognizable by traders such as option prices' partial derivatives with respect to a relevant model and state parameters (the so called Greeks). Let us denote by $\Pi_{k}^{i, \Phi_{i}}(S, \sigma)$ the Black-Scholes price at time $t_{k}$ of the summed payoff $\Phi_{i}\left(S_{i}\right)$ at time $t_{i} \geq t_{k}$ :

$$
\Pi_{k}^{i, \Phi_{i}}(S, \sigma)=e^{-r\left(t_{i}-t_{k}\right)} \mathbf{E}^{\mathbb{Q}}\left[\Phi_{i}\left(S_{i}\right) \mid S_{k}=S\right],
$$

where $\mathbb{Q}$ is the risk-neutral measure of the Black-Scholes model with volatility $\sigma$. We denote by $\operatorname{Bin}_{k}^{i, \Phi_{i}}(S)$ the binomial price at time $t_{k}$ of the summed payoff $\Phi_{i}\left(S_{i}\right)$ at time $t_{i} \geq t_{k}$ :

$$
\operatorname{Bin}_{k}^{i, \Phi_{i}}(S)=e^{-r\left(t_{i}-t_{k}\right)} \mathbf{E}^{\hat{\mathbb{Q}}}\left[\Phi_{i}\left(S_{i}\right) \mid S_{k}=S\right],
$$

$k=0, \ldots, N-1, i=k+1, \ldots, N$, where $\hat{\mathbb{Q}}$ is the risk-neutral measure of the binomial model with probabilities

$$
\begin{aligned}
& \hat{\mathbb{Q}}\left(S_{k+1}=u_{k} S_{k}\right)=q_{k}^{u}=\frac{e^{r \Delta t_{k}}-d_{k}}{u_{k}-d_{k}}, \\
& \hat{\mathbb{Q}}\left(S_{k+1}=d_{k} S_{k}\right)=q_{k}^{d}=\frac{u_{k}-e^{r \Delta t_{k}}}{u_{k}-d_{k}},
\end{aligned}
$$

with $u_{k}$ and $d_{k}$ defined as in (5.2). At the time $t_{k}$ we define Delta, Vega, Vanna and Volga of the payoff at time $t_{i} \geq t_{k}$, i.e. of $\Phi_{i}\left(S_{i}\right)$, as

$$
\begin{aligned}
\hat{\Delta}_{k}^{i} & =\frac{\operatorname{Bin}_{k+1}^{i, \Phi_{i}}\left(S_{k} u_{k}\right)-\operatorname{Bin}_{k+1}^{i, \Phi_{i}}\left(S_{k} d_{k}\right)}{S_{k}\left(u_{k}-d_{k}\right)}, \\
\mathcal{V}_{k}^{i} & =\frac{\partial \prod_{k}^{i, \Phi_{i}}}{\partial \sigma}\left(S_{k}, \sigma\right), \\
\operatorname{vanna}_{k}^{i} & =\frac{\partial^{2} \prod_{k}^{i, \Phi_{i}}}{\partial S \partial \sigma}\left(S_{k}, \sigma\right), \\
\operatorname{volga}_{k}^{i} & =\frac{\partial^{2} \prod_{k}^{i, \Phi_{i}}}{\partial \sigma^{2}}\left(S_{k}, \sigma\right),
\end{aligned}
$$

$k=0, \ldots, N-1, i>k$. Note, that we do not define Delta in the usual way as the derivative of the Black-Scholes price with respect to the underlying price process $\frac{\partial \Pi^{i}}{\partial S}$, but as its binomial equivalent. Pelsser and Vorst [37] show that using the binomial Delta is more accurate than its continuous counterpart. 
Further on we will see that binomial Deltas make sure that the optimal hedging strategy is a corrected binomial rather than Black-Scholes hedge, which in turn provides transparent analytical formulae for the indifference prices. Since the trader is allowed to hedge only with the underlying asset, he or she can only (partially) hedge Delta of his portfolio. The volatility risk can be secured by the reserve bank account but not hedging, therefore we would like to include not only the linear sensitivity of the price to volatility Vega but also the second-order terms Vanna and Volga.

Second, we want our solutions to be bounded. If for some $k=0, \ldots, N-1$ the risk function $\rho_{k}\left(S, V, h^{S}\right)$ is linear in the third parameter, then $\mathbf{E}\left[V_{N}\right]$ is a linear function of $h_{k}^{S}$ (see the dynamics of $V$ in (5.7)). Then the optimal value of $h_{k}^{S}$ for the maximization of $\mathbf{E}\left[V_{N}\right]$ is either plus or minus infinity depending on the sign of the coefficient before $h_{k}^{S}$. Therefore we need $\rho_{k}\left(S_{k}, V_{k}, h_{k}^{S}\right)$ to be a non-linear function of $h_{k}^{S}$ for all $k=0, \ldots, N-1$. This condition excludes, for example, a risk function equal to the Delta or any other Greek of the portfolio, which are linear or do not depend on $h^{S}$.

Thirdly, to make calculations of the optimal strategies easier we require the function $\rho_{k}\left(S_{k}, V_{k}, h_{k}^{S}\right)$ to be convex function of the third variable. Let us remind the reader that $\rho_{k}\left(S_{k}, V_{k}, h_{k}^{S}\right)$ appears in (5.8) with a negative coefficient $E_{k}$, so if $\rho_{k}\left(S_{k}, V_{k}, h_{k}^{S}\right)$ is convex in the third argument then $V_{k+1}$ is a concave function of $h_{k}^{S}$.

And finally we would like our risk function to be positive only for those values of the Greeks that traders do hedge. A positive Volga, for example, means that the price of the portfolio is a convex function of volatility. Therefore changes in volatility will result in higher price growth than price decline. (In some neighborhood of $\mathcal{V}=0$ any change in volatility will result in the portfolio price growth.) Thus traders prefer not to hedge Volga if it is positive. If Vanna is negative and the stock price and the volatility change differently (one drops, while another grows), then again the possible price growth is higher than the possible price decline. In equity markets stock prices and volatility are negatively correlated, which means that often their values move in the opposite directions more often than in the same. That is why equity traders prefer to keep negative Vanna unhedged. For some assets (e.g. some foreign exchange rates) the spot-volatility correlation is positive. In those cases a positive Vanna is left unprotected.

Taking all this into account we decide to use the following risk function:

$$
\begin{gathered}
\rho_{k}\left(S_{k}, h_{k}^{S}\right)=\alpha_{1} S_{k}^{2}\left(h_{k}^{S}+\hat{\Delta}_{k}\right)^{2}+\frac{\left(\mathbf{E}_{k}\left[R_{k}\right]-e^{r \Delta t_{k}}\right)^{2}}{4 \alpha_{1} E_{k}^{2}}+\alpha_{2}\left|\mathcal{V}_{k}\right| \\
+\max \left(\alpha_{3} \operatorname{vanna}_{k}, 0\right)+\max \left(-\alpha_{4} \operatorname{volga}_{k}, 0\right),
\end{gathered}
$$


where $\alpha_{1}, \alpha_{2}, \alpha_{4} \geq 0, \alpha_{3} \in \mathbb{R}$ and $\mathcal{V}_{k}$, vanna , volga $_{k}$ are the sums of the corresponding Black-Scholes Greeks and $\hat{\Delta}_{k}$ is the sum of binomial Deltas of all the options included in the portfolio:

$$
\begin{aligned}
\hat{\Delta}_{k} & =\sum_{i=k+1}^{n} \hat{\Delta}_{k}^{i}, \\
\mathcal{V}_{k} & =\sum_{i=k+1}^{n} \mathcal{V}_{k}^{i}, \\
\operatorname{vanna}_{k} & =\sum_{i=k+1}^{n} \operatorname{vanna}_{k}^{i}, \\
\operatorname{volga}_{k} & =\sum_{i=k+1}^{n} \operatorname{volga}_{k}^{i},
\end{aligned}
$$

$k=0, \ldots, N-1$. Note that unlike other parameters $\alpha_{3}$ may be either positive or negative. We may choose its sign according to the correlation of the volatility and the underlying. The second term is there to make sure that the model does not produce arbitrage. Actually, as it will be clear further on, this term is equal to the expected profit on the $k$ th step from the optimal trading in the underlying asset, divided by $-E_{k}$. So it eliminates the average growth of the portfolio value due to the trading in the underlying market.

Unlike VaR the risk function $\rho_{k}$ does not depend on the distribution of the underlying assets but depends on observable parameters only. It measures the local risk of a trader in each particular state of the market. Note, that it is not dependent on the portfolio value. Each parameter $\alpha_{j},(j=2,3,4)$ can be thought of as a market price of risk we assign based on the $j$ th Greek. The risk contained in the Greeks cannot be hedged by trading in the underlying asset, except for Delta. Including these Greeks in the risk function affects the indifference prices and creates bid-ask spreads (while not affecting the hedging strategies). In section 5.3 we derive an analytical formula for the indifference prices, which implies that they are not affected by the coefficient $\alpha_{1}$. This coefficient is a risk aversion parameter, which reflects how well we wish to be delta-hedged. The analytical formula also implies that $\alpha_{2}$ does not affect the mid prices in the presence of the zero portfolio due to the even appearance of Vega in the function $\rho_{k}\left(S, h^{S}\right)$.

From now on we will use the following notation:

$$
\hat{\rho}_{k}\left(S_{k}\right)=\alpha_{2}\left|\mathcal{V}_{k}\right|+\max \left(\alpha_{3} \operatorname{vanna}_{k}, 0\right)+\max \left(-\alpha_{4} \operatorname{volga}_{k}, 0\right)
$$


for all $k=0, \ldots, N-1$, so

$$
\rho_{k}\left(S_{k}, h_{k}^{S}\right)=\alpha_{1} S_{k}^{2}\left(h_{k}^{S}+\hat{\Delta}_{k}\right)^{2}+\frac{\left(\mathbf{E}\left[R_{k}\right]-e^{r \Delta t_{k}}\right)^{2}}{4 \alpha_{1} E_{k}^{2}}+\hat{\rho}_{k}\left(S_{k}\right)
$$

for all $k=0, \ldots, N-1$.

\subsection{The Dynamic Programming Algorithm}

This section provides the algorithm for the numerical solution of our optimization problem (5.7). By theorem 4.2.1 to find the solution we should optimize by proceeding backwards step by step, starting in $t_{N}$ :

$$
\begin{aligned}
J_{N}\left(S_{N}, V_{N}\right)= & V_{N} \\
J_{k}\left(S_{k}, V_{k}\right)= & \max _{h_{k}^{S}} \mathbf{E}_{k}\left[J _ { k + 1 } \left(S_{k} R_{k}, V_{k} e^{r \Delta t_{k}}+S_{k} h_{k}^{S}\left(R_{k}-e^{r \Delta t_{k}}\right)\right.\right. \\
& \left.\left.\quad+\rho_{k}\left(S_{k}, h_{k}^{S}\right) E_{k}+\Phi_{k+1}\left(S_{k} R_{k}\right)\right)\right]
\end{aligned}
$$

for all $k=0, \ldots, N-1$. Note that here we optimize as in chapter 4 , over Markov policies without loss of generality. The following proposition states that $J_{k}(S, V)$ defined by (5.16) is an affine function of $V$ :

Proposition 5.2.1 The $J_{k}(S, V)$ as defined in (5.16) are affine functions of $V$ :

$$
J_{k}(S, V)=A_{k} V+C_{k}(S), \quad k=0, \ldots, N,
$$

where $A_{k}=e^{r\left(T-t_{k}\right)}$ and $C_{k}: \mathbb{R}^{+} \rightarrow \mathbb{R}$ is a deterministic function that does not depend on $V$.

Proof We prove the proposition by backward induction in $k$. First, the statement is true for $k=N$ :

$$
\begin{aligned}
& A_{N}=1 \\
& C_{N}=0
\end{aligned}
$$

Assume the statement is true for $k+1, \ldots, N$. Let us prove the statement for $k$. Using (5.16) and the induction hypothesis we have

$$
\begin{aligned}
J_{k}\left(S_{k}, V_{k}\right)= & \max _{h_{k}^{S}} \mathbf{E}_{k}\left[A _ { k + 1 } \cdot \left\{V_{k} e^{r \Delta t_{k}}+S_{k} h_{k}^{S}\left(R_{k}-e^{r \Delta t_{k}}\right)\right.\right. \\
& \left.\left.\quad+\rho_{k}\left(S_{k}, h_{k}^{S}\right) E_{k}+\Phi_{k+1}\left(S_{k} R_{k}\right)\right\}+C_{k+1}\left(S_{k} R_{k}\right)\right] \\
= & \max _{h_{k}^{S}}\left[A_{k+1} e^{r \Delta t_{k}} V_{k}+A_{k+1}\left\{S_{k} h_{k}^{S}\left(\mathbf{E}_{k}\left[R_{k}\right]-e^{r \Delta t_{k}}\right)\right.\right. \\
& \left.\left.\quad+\rho_{k}\left(S_{k}, h_{k}^{S}\right) E_{k}+\mathbf{E}_{k}\left[\Phi_{k+1}\left(S_{k} R_{k}\right)\right]\right\}+\mathbf{E}_{k}\left[C_{k+1}\left(S_{k} R_{k}\right)\right]\right] .
\end{aligned}
$$


Since $\rho_{k}\left(S_{k}, h_{k}^{S}\right)$ is a convex quadratic function of the second argument (see (5.15) and (5.14)), then $\rho_{k}\left(S_{k}, h_{k}^{S}\right) E_{k}$ is quadratic with a negative highest order coefficient, because $E_{k}<0$, and $J_{k}\left(S_{k}, V_{k}\right)$ is the maximum of a quadratic function of $h_{k}^{S}$ with a positive highest order coefficient. Therefore

$$
\begin{aligned}
J_{k}\left(S_{k}, V_{k}\right)= & A_{k+1} e^{r \Delta t_{k}} V_{k}+\left(A _ { k + 1 } \left\{S_{k}\left(h_{k}^{S}\right)^{*}\left(\mathbf{E}_{k}\left[R_{k}\right]-e^{r \Delta t_{k}}\right)\right.\right. \\
& +\left(\alpha_{1} S_{k}^{2}\left(\left(h_{k}^{S}\right)^{*}+\hat{\Delta}_{k}\right)^{2}+\frac{\left(\mathbf{E}\left[R_{k}\right]-e^{r \Delta t_{k}}\right)^{2}}{4 \alpha_{1} E_{k}^{2}}+\hat{\rho}_{k}\left(S_{k}\right)\right) E_{k} \\
& \left.\left.+\mathbf{E}_{k}\left[\Phi_{k+1}\left(S_{k} R_{k}\right)\right]\right\}+\mathbf{E}_{k}\left[C_{k+1}\left(S_{k} R_{k}\right)\right]\right)
\end{aligned}
$$

with

$$
\left(h_{k}^{S}\right)^{*}=-\hat{\Delta}_{k}-\frac{\left(\mathbf{E}_{k}\left[R_{k}\right]-e^{r \Delta t_{k}}\right)}{2 \alpha_{1} S_{k} E_{k}} .
$$

This means that $J_{k}\left(S_{k}, V_{k}\right)$ is also a linear function of $V_{k}$ :

$$
J_{k}\left(S_{k}, V_{k}\right)=A_{k} V_{k}+B_{k}\left(S_{k}\right)
$$

where we take

$$
\begin{aligned}
A_{k}= & A_{k+1} e^{r \Delta t_{k}} \\
C_{k}\left(S_{k}\right)= & A_{k+1}\left\{-S_{k} \hat{\Delta}_{k}\left(\mathbf{E}_{k}\left[R_{k}\right]-e^{r \Delta t_{k}}\right)+\hat{\rho}_{k}\left(S_{k}\right) E_{k}\right. \\
& \left.\quad+\mathbf{E}_{k}\left[\Phi_{k+1}\left(S_{k} R_{k}\right)\right]\right\}+\mathbf{E}_{k}\left[C_{k+1}\left(S_{k} R_{k}\right)\right]
\end{aligned}
$$

Note that equation (5.17) gives us the optimal hedging strategy. For small time increments we have the following asymptotics for $k=0, \ldots, N-1$

$$
\left(h_{k}^{S}\right)^{*} S_{k}=-\hat{\Delta}_{k} S_{k}+e^{-\tilde{r} t_{k}}\left(\frac{\mu-r}{r-\tilde{r}}\right) \frac{1}{2 \alpha_{1}}+o(1), \Delta t_{k} \rightarrow 0
$$

So the optimal strategy is a corrected binomial hedge. The asymptotics of the correction term show that the bigger is the difference between the drift $\mu$ and the risk-free rate $r$ the more we should invest in the underlying asset. On the other hand, the bigger the difference between the rates on the reserve and the regular bank accounts and the higher the value of the risk aversion parameter $\alpha_{1}$, the less we should invest in the underlying.

Proposition 5.2.1 provides us with formulae for the numerical algorithm. We run the backward recursion for $A_{k}$ and $C_{k}(S)$ using (5.18) to get $A_{0}=e^{r T}$ and $C_{0}(S)$. By Theorem 4.2.1, $J_{0}\left(S_{0}, V_{0}\right)=e^{r T} V_{0}+C_{0}\left(S_{0}\right)$ is then the solution 
to our optimization problem, and it is the expected value of the optimally hedged portfolio at time $T$. $C_{0}\left(S_{0}\right)$ is the effect of the introduction of options in the portfolio. Note, that if there are no options (payoff function $\Phi(t, S)$ is zero for all $\left.t \in \mathcal{T}, S \in \mathbb{R}^{+}\right)$, then $C_{0}\left(S_{0}\right)$ is also zero and $J_{0}\left(S_{0}, V_{0}\right)=e^{r T} V_{0}$, the value of investing all initial capital in the bank account $B$. Note that the extra investment in stocks does not change the expected value, because the second term of the risk function (see (5.15)) nullifies it.

\subsection{Analytical Solutions for the Indifference Prices}

In this section we prove a theorem which gives a relation between the indifference price and the no-arbitrage price in the binomial model. This result gives a clear characterization of the prices in the model with cost of risk as the binomial price plus a correction term dependent on the risk function. The fact that indifference prices do not depend on the parameter $\alpha_{1}$ and that the indifference mid prices do not depend on the parameter $\alpha_{2}$ are then the corollaries. We also prove that the buying indifference price of a portfolio is the minimum of all its selling prices.

Further we will add superscripts $\Phi, \Psi$ etc. to some functions to stress the relation of these functions to portfolios $\Phi, \Psi$ etc. For example we write $\rho_{k}^{\Phi}\left(S_{k}\right)$ for the value of the risk function of portfolio $\Phi$ and $\hat{\Delta}_{k}^{i, \Phi}$ for the Delta at time $t_{k}$ of the total payoff $\Phi_{i}$ at time $t_{i}>t_{k}$ of option portfolio $\Phi$. We define

$$
E_{k, i}=e^{\tilde{r} t_{i}-r\left(t_{i+1}-t_{k}\right)}\left(e^{\tilde{r} \Delta t_{i}}-e^{r \Delta t_{i}}\right)
$$

for all $k=0, \ldots, N-1$ and $i=k, \ldots, N-1$.

Theorem 5.3.1 For all $k=0, \ldots, N$ and all portfolios $\Phi$ and $\Psi$ of European options the following equations hold

$$
\begin{aligned}
& \{\Phi\}_{\{\Psi\}, k}^{s}\left(S_{k}\right)=\sum_{i=k+1}^{N} \operatorname{Bin}_{k}^{i, \Phi_{i}}\left(S_{k}\right)+\sum_{i=k}^{N-1} \boldsymbol{E}_{k}\left[\hat{\rho}_{i}^{\Psi}\left(S_{i}\right)-\hat{\rho}_{i}^{\Psi-\Phi}\left(S_{i}\right)\right] E_{k, i} \\
& \{\Phi\}_{\{\Psi\}, k}^{b}\left(S_{k}\right)=\sum_{i=k+1}^{N} \operatorname{Bin}_{k}^{i, \Phi_{i}}\left(S_{k}\right)+\sum_{i=k}^{N-1} \boldsymbol{E}_{k}\left[\hat{\rho}_{i}^{\Phi+\Psi}\left(S_{i}\right)-\hat{\rho}_{i}^{\Psi}\left(S_{i}\right)\right] E_{k, i},
\end{aligned}
$$

where $\hat{\rho}_{k}(S)$ is defined in (5.14).

Before proving the theorem we prove the following two lemmas. 
Lemma 5.3.2 Let us for all $k=0, \ldots, N-1$ and for any measurable function $f: \mathbb{R} \rightarrow \mathbb{R}$ define $\tilde{\Delta}_{k}^{f}$ as

$$
\tilde{\Delta}_{k}^{f}(S)=\frac{f\left(S u_{k}\right)-f\left(S d_{k}\right)}{S u_{k}-S d_{k}}
$$

Then the following equation holds

$$
\boldsymbol{E}_{k}\left[f\left(S_{k} R_{k}\right)\right]-S_{k} \tilde{\Delta}_{k}^{f}\left(S_{k}\right)\left(\boldsymbol{E}_{k}\left[R_{k}\right]-e^{r \Delta t_{k}}\right)=\boldsymbol{E}_{k}^{\hat{\mathbb{Q}}}\left[f\left(S_{k} R_{k}\right)\right],
$$

where $\tilde{\mathbb{Q}}$ is defined in (5.9)-(5.10).

\section{Proof}

$$
\begin{aligned}
\mathbf{E}_{k} & {\left[f\left(S_{k} R_{k}\right)\right]-S_{k} \tilde{\Delta}_{k}^{f}\left(\mathbf{E}_{k}\left[R_{k}\right]-e^{r \Delta t_{k}}\right) } \\
& =\frac{f\left(S_{k} u_{k}\right)+f\left(S_{k} d_{k}\right)}{2}-S_{k} \frac{f\left(S_{k} u_{k}\right)-f\left(S_{k} d_{k}\right)}{S_{k} u_{k}-S_{k} d_{k}}\left(\frac{u_{k}+d_{k}}{2}-e^{r \Delta t_{k}}\right) \\
& =f\left(S_{k} u_{k}\right) \frac{e^{r \Delta t_{k}}-d_{k}}{u_{k}-d_{k}}+f\left(S_{k} d_{k}\right) \frac{u_{k}-e^{r \Delta t_{k}}}{u_{k}-d_{k}} \\
& =\mathbf{E}_{k}^{\mathbb{Q}}\left[f\left(S_{k} R_{k}\right)\right] .
\end{aligned}
$$

Note that if $f$ is the binomial price at time $t_{k}$ of the payoff $\Phi_{i}\left(S_{i}\right)$ at time $t_{i}>t_{k}$

$$
f(S)=\operatorname{Bin}_{k+1}^{i, \Phi_{i}}(S)
$$

then

$$
\tilde{\Delta}_{k}^{f}\left(S_{k}\right)=\hat{\Delta}_{k}^{i, \Phi}
$$

defined in (5.11). Thus by lemma 5.3.2,

$$
\mathbf{E}_{k}\left[\operatorname{Bin}_{k+1}^{i, \Phi_{i}}\left(S_{k} R_{k}\right)\right]-S_{k} \hat{\Delta}_{k}^{i, \Phi}\left(\mathbf{E}_{k}\left[R_{k}\right]-e^{r \Delta t_{k}}\right)=\mathbf{E}_{k}^{\hat{\mathbb{Q}}}\left[\operatorname{Bin}_{k+1}^{i, \Phi_{i}}\left(S_{k} R_{k}\right)\right]
$$

Lemma 5.3.3 For all $k=0, \ldots, N$ and all portfolios $\Phi$ and $\Psi$ of European options

$$
\begin{aligned}
& \{\Phi\}_{\{\Psi\}, k}^{s}\left(S_{k}\right)=e^{-r\left(T-t_{k}\right)}\left(C_{k}^{\Psi}\left(S_{k}\right)-C_{k}^{\Psi-\Phi}\left(S_{k}\right)\right), \\
& \{\Phi\}_{\{\Psi\}, k}^{b}\left(S_{k}\right)=e^{-r\left(T-t_{k}\right)}\left(C_{k}^{\Psi+\Phi}\left(S_{k}\right)-C_{k}^{\Psi}\left(S_{k}\right)\right),
\end{aligned}
$$

where the functions $C_{k}$ are as defined in (5.18). 
Proof We prove only the first equality since the proof for the second one is similar. By definition 5.1.2 and Theorem 4.2.1 the indifference selling price at time $t_{k}$ is

$$
\{\Phi\}_{\{\Psi\}, k}^{s}\left(S_{k}\right)=e^{-r\left(T-t_{k}\right)}\left(J_{k}^{\Psi}\left(S_{k}, V_{k}\right)-J_{k}^{\Psi-\Phi}\left(S_{k}, V_{k}\right)\right),
$$

where

$$
\begin{aligned}
J_{k}^{\Psi}\left(S_{k}, V_{k}\right) & =A_{k} V_{k}+C_{k}^{\Psi}\left(S_{k}\right) \\
J_{k}^{\Psi-\Phi}\left(S_{k}, V_{k}\right) & =A_{k} V_{k}+C_{k}^{\Psi-\Phi}\left(S_{k}\right)
\end{aligned}
$$

therefore

$$
\{\Phi\}_{\{\Psi\} k}^{s}\left(S_{k}\right)=e^{-r\left(T-t_{k}\right)}\left(C_{k}^{\Psi}\left(S_{k}\right)-C_{k}^{\Psi-\Phi}\left(S_{k}\right)\right)
$$

Now we are ready to prove Theorem 5.3.1.

Proof (of Theorem 5.3.1). We prove (5.20) by backward induction in $k$.

1. The case of $k=N$ is trivial, since both sides of the equality are zeros:

$$
\begin{aligned}
\{\Phi\}_{\{\Psi\}, N}^{s}\left(S_{N}\right)=J_{N}^{\Psi}\left(S_{N}, V_{N}\right)-J_{N}^{\Psi-\Phi}\left(S_{N}, V_{N}\right)=V_{N}-V_{N} & =0 \\
\sum_{i=N+1}^{N} \operatorname{Bin}_{N}^{\Phi_{i}}\left(S_{N}\right)+\sum_{i=N}^{N-1} \mathbf{E}_{k}\left[\hat{\rho}^{\Psi}\left(S_{i}\right)-\hat{\rho}^{\Psi-\Phi}\left(S_{i}\right)\right] e^{\tilde{r} t_{i}\left(e^{\tilde{r} \Delta t_{i}}-e^{r \Delta t_{i}}\right)} & =0
\end{aligned}
$$

2. Assume we have proved (5.20) for all $k+1, \ldots, N$. Now we prove it for $k$. From (5.23) we have

$$
\{\Phi\}_{\{\Psi\}, k}^{s}\left(S_{k}\right)=e^{-r\left(T-t_{k}\right)}\left(C_{k}^{\Psi}\left(S_{k}\right)-C_{k}^{\Psi-\Phi}\left(S_{k}\right)\right),
$$

substituting $C_{k}\left(S_{k}\right)$ from (5.18) we write

$$
\begin{aligned}
\{\Phi\}_{\{\Psi\}, k}^{s}\left(S_{k}\right)=e^{-r \Delta t_{k}}\left\{-S_{k}\left(\hat{\Delta}_{k}^{\Psi}-\hat{\Delta}_{k}^{\Psi-\Phi}\right)\left(\mathbf{E}_{k}\left[R_{k}\right]-e^{r \Delta t_{k}}\right)\right. \\
+\left(\hat{\rho}_{k}^{\Psi}\left(S_{k}\right)-\hat{\rho}_{k}^{\Psi-\Phi}\left(S_{k}\right)\right) E_{k}+\mathbf{E}_{k}\left[\Psi_{k+1}\left(S_{k} R_{k}\right)\right] \\
\left.-\mathbf{E}_{k}\left[\Psi_{k+1}\left(S_{k} R_{k}\right)-\Phi_{k+1}\left(S_{k} R_{k}\right)\right]\right\} \\
+e^{-r\left(T-t_{k}\right)}\left\{\mathbf{E}_{k}\left[C_{k+1}^{\Psi}\left(S_{k} R_{k}\right)\right]-\mathbf{E}_{k}\left[C_{k+1}^{\Psi-\Phi}\left(S_{k} R_{k}\right)\right]\right\} .
\end{aligned}
$$

By the definition of the portfolio Delta (5.13) and by (5.23) we write

$$
\begin{aligned}
\{\Phi\}_{\{\Psi\}, k}^{s}\left(S_{k}\right)=e^{-r \Delta t_{k}}\left\{-S_{k} \sum_{i=k+1}^{N} \hat{\Delta}_{k}^{i, \Phi}\left(\mathbf{E}_{k}\left[R_{k}\right]-e^{r \Delta t_{k}}\right)\right. \\
\\
+\left(\hat{\rho}_{k}^{\Psi}\left(S_{k}\right)-\hat{\rho}_{k}^{\Psi-\Phi}\left(S_{k}\right)\right) E_{k}+\mathbf{E}_{k}\left[\Phi_{k+1}\left(S_{k} R_{k}\right)\right] \\
\left.+\mathbf{E}_{k}\left[\{\Phi\}_{\{\Psi\}, k+1}^{s}\left(S_{k} R_{k}\right)\right]\right\} .
\end{aligned}
$$


By the induction assumption

$\{\Phi\}_{\{\Psi\}, k+1}^{s}\left(S_{k} R_{k}\right)=\sum_{i=k+2}^{N} \operatorname{Bin}_{k+1}^{i, \Phi_{i}}\left(S_{k} R_{k}\right)+\sum_{i=k+1}^{N-1} \mathbf{E}_{k+1}\left[\hat{\rho}_{i}^{\Psi}\left(S_{i}\right)-\hat{\rho}_{i}^{\Psi-\Phi}\left(S_{i}\right)\right] E_{k+1, i}$,

therefore

$$
\begin{aligned}
\{\Phi\}_{\{\Psi\}, k}^{s}\left(S_{k}\right)= & e^{-r \Delta t_{k}}\left\{-S_{k} \sum_{i=k+1}^{N} \hat{\Delta}_{k}^{i, \Phi_{i}}\left(\mathbf{E}_{k}\left[R_{k}\right]-e^{r \Delta t_{k}}\right)\right. \\
& +\left(\hat{\rho}_{k}^{\Psi}\left(S_{k}\right)-\hat{\rho}_{k}^{\Psi-\Phi}\left(S_{k}\right)\right) E_{k} \\
& +\mathbf{E}_{k}\left[\Phi_{k+1}\left(S_{k} R_{k}\right)\right]+\sum_{i=k+2}^{N} \mathbf{E}_{k}\left[\operatorname{Bin}_{k+1}^{i, \Phi_{i}}\left(S_{k} R_{k}\right)\right] \\
& \left.+\sum_{i=k+1}^{N-1} \mathbf{E}_{k}\left[\hat{\rho}_{i}^{\Psi}\left(S_{i}\right)-\hat{\rho}_{i}^{\Psi-\Phi}\left(S_{i}\right)\right] E_{k+1, i}\right\} .
\end{aligned}
$$

Regrouping the terms we get

$$
\begin{aligned}
\{\Phi\}_{\{\Psi\}, k}^{s}\left(S_{k}\right)=e^{-r \Delta t_{k}}\left\{\mathbf{E}_{k}\left[\Phi_{k+1}\left(S_{k} R_{k}\right)\right]-S_{k} \hat{\Delta}_{k}^{k+1, \Phi_{k+1}}\left(\mathbf{E}_{k}\left[R_{k}\right]-e^{r \Delta t_{k}}\right)\right. \\
+\sum_{i=k+2}^{N} \mathbf{E}_{k}\left[\operatorname{Bin}_{k+1}^{i, \Phi_{i}}\left(S_{k} R_{k}\right)\right]-S_{k} \sum_{i=k+2}^{N} \hat{\Delta}_{k}^{i, \Phi_{i}}\left(\mathbf{E}_{k}\left[R_{k}\right]-e^{r \Delta t_{k}}\right) \\
\left.+\sum_{i=k}^{N-1} \mathbf{E}_{k}\left[\hat{\rho}_{i}^{\Psi}\left(S_{i}\right)-\hat{\rho}_{i}^{\Psi-\Phi}\left(S_{i}\right)\right] E_{k+1, i}\right\},
\end{aligned}
$$

which by Lemma 5.3.2 and (5.22) yields

$$
\begin{aligned}
\{\Phi\}_{\{\Psi\}, k}^{s}\left(S_{k}\right)= & e^{-r \Delta t_{k}}\left\{\mathbf{E}_{k}^{\hat{\mathbb{Q}}}\left[\Phi_{k+1}\left(S_{k} R_{k}\right)\right]+\sum_{i=k+2}^{N} \mathbf{E}_{k}^{\hat{\mathbb{Q}}}\left[\operatorname{Bin}_{k+1}^{i, \Phi_{i}}\left(S_{k} R_{k}\right)\right]\right\} \\
& \quad+\sum_{i=k}^{N-1} \mathbf{E}_{k}\left[\hat{\rho}_{i}^{\Psi}\left(S_{i}\right)-\hat{\rho}_{i}^{\Psi-\Phi}\left(S_{i}\right)\right] E_{k, i} \\
= & \sum_{i=k+1}^{N} \operatorname{Bin}_{k}^{i, \Phi_{i}}\left(S_{k}\right)+\sum_{i=k}^{N-1} \mathbf{E}_{k}\left[\hat{\rho}_{i}^{\Psi}\left(S_{i}\right)-\hat{\rho}_{i}^{\Psi-\Phi}\left(S_{i}\right)\right] E_{k, i}
\end{aligned}
$$

and thus we have proved (5.20). The proof of (5.21) is similar.

As a straightforward corollary from this theorem follow the independence of the indifference prices of the parameter $\alpha_{1}$ and the independence of the mid indifference prices in the presence of the zero portfolio of the parameter $\alpha_{2}$. 
Corollary 5.3.4 For all portfolios $\Phi$ and $\Psi$, for all $k=0, \ldots, N-1$ (i) the indifference prices $\{\Phi\}_{\{\Psi\}, k}^{s},\{\Phi\}_{\{\Psi\}, k}^{b}$ and $\{\Phi\}_{\{\Psi\}, k}^{m}$ do not depend on $\alpha_{1}$,

(ii) the indifference mid prices in presence of the zero portfolio $\{\Phi\}_{\{0\}, k}^{m}$ do not depend on $\alpha_{2}$.

Proof (i) Since $\hat{\rho}_{k}^{\Phi}(S)$ is independent of $\alpha_{1}$ for all $k=0, \ldots, N-1$ and all portfolios $\Phi$, then by (5.20) and (5.21) $\{\Phi\}_{\{\Psi\}, k}^{s}$ and $\{\Phi\}_{\{\Psi\}, k}^{b}$ are also independent of $\alpha_{1}$, therefore $\{\Phi\}_{\{\Psi\}, k}^{m}$ is also independent.

(ii) By definition

$$
\{\Phi\}_{\{0\}, k}^{m}=\frac{\{\Phi\}_{\{0\}, k}^{s}+\{\Phi\}_{\{0\}, k}^{b}}{2}
$$

Therefore by Theorem 5.3.1

$$
\{\Phi\}_{\{0\}, k}^{m}=\sum_{i=k+1}^{N} \operatorname{Bin}_{k}^{i, \Phi_{i}}\left(S_{k}\right)+\sum_{i=k}^{N-1} \mathbf{E}_{k}\left[\frac{\hat{\rho}_{i}^{\Phi}\left(S_{i}\right)-\hat{\rho}_{i}^{-\Phi}\left(S_{i}\right)}{2}\right] E_{k, i} .
$$

By the definition of $\hat{\rho}_{k}\left(S_{k}\right)$ from (5.14) we write for all $i=k, \ldots, N-1$

$$
\begin{aligned}
\hat{\rho}_{i}^{\Phi}\left(S_{i}\right)-\hat{\rho}_{i}^{-\Phi}\left(S_{i}\right)=\alpha_{2} & \left(\left|\mathcal{V}_{i}^{\Phi}\right|-\left|\mathcal{V}_{i}^{-\Phi}\right|\right) \\
& +\left(\max \left(\alpha_{3} \operatorname{vanna}_{i}^{\Phi}, 0\right)-\max \left(\alpha_{3} \operatorname{vanna}_{i}^{-\Phi}, 0\right)\right) \\
& +\left(\max \left(-\alpha_{4} \operatorname{volga}_{i}^{\Phi}, 0\right)-\max \left(-\alpha_{4} \operatorname{volga}_{i}^{-\Phi}, 0\right)\right) \\
=\alpha_{2} & \left(\left|\mathcal{V}_{i}^{\Phi}\right|-\left|-\mathcal{V}_{i}^{\Phi}\right|\right) \\
& +\left(\max \left(\alpha_{3} \operatorname{vanna}_{i}^{\Phi}, 0\right)-\max \left(-\alpha_{3} \operatorname{vanna}_{i}^{\Phi}, 0\right)\right) \\
& +\left(\max \left(-\alpha_{4} \operatorname{volga}_{i}^{\Phi}, 0\right)-\max \left(\alpha_{4} \operatorname{volga}_{i}^{\Phi}, 0\right)\right) \\
= & \alpha_{3} \operatorname{vanna}_{i}^{\Phi}-\alpha_{4} \operatorname{volga}_{i}^{\Phi} .
\end{aligned}
$$

Hence $\{\Phi\}_{\{0\}, k}^{m}$ does not depend on $\alpha_{2}$.

In the market all options are available to buy for their selling prices. Another interesting property of the model with cost of risk is that for any options portfolio $\Phi$ its buying indifference price in the presence of the zero portfolio is the smallest selling indifference prices of this portfolio.

Theorem 5.3.5 For all $k=0, \ldots, N-1$, all portfolios of European options $\Phi$, and all $S \in \mathbb{R}^{+}$the following is true:

$$
\{\Phi\}_{\{0\}, k}^{b}(S)=\min _{\Psi}\left\{\{\Phi\}_{\{\Psi\}, k}^{s}(S)\right\} .
$$


Proof First we note that for all $k=0, \ldots, N-1, \beta>0, S \in \mathbb{R}^{+}$and any portfolio of European options $\Phi$

$$
\hat{\rho}_{k}^{\beta \Phi}(S)=\beta \hat{\rho}_{k}^{\Phi}(S) .
$$

Indeed,

$$
\begin{aligned}
\hat{\rho}_{k}^{\beta \Phi}(S) & =\alpha_{2}\left|\mathcal{V}_{k}^{\beta \Phi}\right|+\max \left(\alpha_{3} \operatorname{vanna}_{k}^{\beta \Phi}, 0\right)+\max \left(-\alpha_{4} \operatorname{volga}_{k}^{\beta \Phi}, 0\right) \\
& =\alpha_{2}\left|\beta \mathcal{V}_{k}^{\Phi}\right|+\max \left(\alpha_{3} \beta \operatorname{vanna}_{k}^{\Phi}, 0\right)+\max \left(-\alpha_{4} \beta \operatorname{volga}_{k}^{\Phi}, 0\right) \\
& =\beta\left(\alpha_{2}\left|\mathcal{V}_{k}^{\Phi}\right|+\max \left(\alpha_{3} \operatorname{vanna}_{k}^{\Phi}, 0\right)+\max \left(-\alpha_{4} \operatorname{volga}_{k}^{\Phi}, 0\right)\right) \\
& =\beta \hat{\rho}_{k}^{\Phi}(S) .
\end{aligned}
$$

We also note, that for all $k=0, \ldots, N-1$, and all $S \in \mathbb{R}^{+}, \hat{\rho}_{k}^{\Phi}(S)$ is a convex function of $\Phi$ :

$$
\hat{\rho}_{k}^{\beta \Phi+(1-\beta) \Psi}(S) \leq \beta \hat{\rho}_{k}^{\Phi}(S)+(1-\beta) \hat{\rho}_{k}^{\Psi}(S)
$$

for any $\beta \in[0,1]$ and $\Phi$ and $\Psi$ portfolios of European options. To see this we write

$$
\begin{aligned}
\hat{\rho}_{k}^{\beta \Phi+} & (1-\beta) \Psi \\
& +\max \left(-\alpha_{4} \operatorname{volga}_{k}^{\beta \Phi+(1-\beta) \Psi}, 0\right) \\
& +\alpha_{2}\left|\mathcal{V}_{k}^{\beta \Phi+(1-\beta) \Psi}\right|+\max \left(\alpha_{3} \operatorname{vanna}_{k}^{\beta \Phi+(1-\beta) \Psi}, 0\right) \\
= & \alpha_{2}\left|\beta \mathcal{V}_{k}^{\Phi}+(1-\beta) \mathcal{V}_{k}^{\Psi}\right|+\max \left(\alpha_{3}\left(\beta \operatorname{vanna}_{k}^{\Phi}+(1-\beta) \operatorname{vanna}_{k}^{\Psi}\right), 0\right) \\
& +\max \left(-\alpha_{4}\left(\beta \operatorname{volga}_{k}^{\Phi}+(1-\beta) \operatorname{volga}_{k}^{\Psi}\right), 0\right) \\
\leq & \beta\left(\alpha_{2}\left|\mathcal{V}_{k}^{\Phi}\right|+\max \left(\alpha_{3} \operatorname{vanna}{ }_{k}^{\Phi}, 0\right)+\max \left(-\alpha_{4} \operatorname{volga}_{k}^{\Phi}, 0\right)\right) \\
& +(1-\beta)\left(\alpha_{2}\left|\mathcal{V}_{k}^{\Psi}\right|+\max \left(\alpha_{3} \operatorname{vanna}_{k}^{\Psi}, 0\right)+\max \left(-\alpha_{4} \operatorname{volga}_{k}^{\Psi}, 0\right)\right) \\
= & \beta \hat{\rho}_{k}^{\Phi}(S)+(1-\beta) \hat{\rho}_{k}^{\Psi}
\end{aligned}
$$

Now using properties (5.26) and (5.27) for all $k=0, \ldots, N-1, S \in \mathbb{R}^{+}$and portfolios $\Phi, \Psi$ we can write

$$
\hat{\rho}_{k}^{\Psi}(S)=\hat{\rho}_{k}^{\Psi-\Phi+\Phi}(S)=2 \hat{\rho}_{k}^{\frac{1}{2}(\Psi-\Phi)+\frac{1}{2} \Phi}(S) \leq \hat{\rho}_{k}^{\Psi-\Phi}(S)+\hat{\rho}_{k}^{\Phi}(S)
$$

so for all $k=0, \ldots, N-1, S \in \mathbb{R}^{+}$and portfolios $\Phi, \Psi$

$$
\hat{\rho}_{k}^{\Psi}(S)-\hat{\rho}_{k}^{\Psi-\Phi}(S) \leq \hat{\rho}_{k}^{\Phi}(S)
$$


By Theorem 5.3.1, noting that $\hat{\rho}_{k}^{0}(S)=0$ for all $k=0, \ldots, N-1, S_{0} \in \mathbb{R}^{+}$ and remembering that since $\tilde{r}<r$ the expression $e^{\tilde{r} \Delta t_{i}}-e^{r \Delta t_{i}}<0$

$$
\begin{aligned}
\{\Phi\}_{\{0\}, k}^{b}\left(S_{k}\right)= & \sum_{i=k+1}^{N} \operatorname{Bin}_{k}^{i, \Phi_{i}}\left(S_{k}\right)+\sum_{i=k}^{N-1} \mathbf{E}_{k}\left[\hat{\rho}_{i}^{\Phi}\left(S_{i}\right)\right] e^{\tilde{r}\left(t_{k}-\Delta t_{i}\right)}\left(e^{\tilde{r} \Delta t_{i}}-e^{r \Delta t_{i}}\right) \\
\leq & \sum_{i=k+1}^{N} \operatorname{Bin}_{k}^{i, \Phi_{i}}\left(S_{k}\right) \\
& \quad+\sum_{i=k}^{N-1} \mathbf{E}_{k}\left[\hat{\rho}_{i}^{\Psi}\left(S_{i}\right)-\hat{\rho}_{i}^{\Psi-\Phi}\left(S_{i}\right)\right] e^{\tilde{r}\left(t_{k}-\Delta t_{i}\right)}\left(e^{\tilde{r} \Delta t_{i}}-e^{r \Delta t_{i}}\right) \\
= & \{\Phi\}_{\{\Psi\}, k}^{s}\left(S_{k}\right),
\end{aligned}
$$

therefore for all $\Psi$, for all $k=0, \ldots, N-1$ and for all $S \in \mathbb{R}^{+}$

$$
\{\Phi\}_{\{0\}}^{b}(S) \leq\{\Phi\}_{\{\Psi\}}^{s}(S) .
$$

The equality holds for $\Psi=\Phi$ :

$$
\begin{aligned}
\{\Phi\}_{\{\Phi\}, k}^{s}(S) & =e^{-r\left(T-t_{k}\right)}\left(C_{k}^{\Phi}(S)-C_{k}^{\Phi-\Phi}(S)\right) \\
& =e^{-r\left(T-t_{k}\right)}\left(C_{k}^{\Phi}(S)-C_{k}^{0}(S)\right)=\{\Phi\}_{\{0\}, k}^{b}(S) .
\end{aligned}
$$

\subsection{Absence of Arbitrage}

In this paragraph we are going to show that our model does not allow arbitrage. We think of arbitrage as an opportunity for a new player in the market to make a sure profit without any initial investments. Or in other words, a possibility for a trader to get a riskless positive profit for a zero initial price when starting from a zero portfolio. Further on in this paragraph we will call a hedging portfolio for options $\Phi$ of a new trader the hedging portfolio of a trader who hedges $\Phi$ and only $\Phi$ optimally according to (5.17). The value of this portfolio at time $t_{k}$ is given by

$$
\begin{gathered}
V_{k+1}=V_{k} e^{r \Delta t}-S_{k} \hat{\Delta}_{k}\left(R_{k}-e^{r \Delta t_{k}}\right)-\frac{\left(R_{k}-e^{r \Delta t_{k}}\right)\left(\mathbf{E}\left[R_{k}\right]-e^{r \Delta t_{k}}\right)}{2 \alpha_{1} E_{k}} \\
+\frac{\left(\mathbf{E}\left[R_{k}\right]-e^{r \Delta t_{k}}\right)^{2}}{2 \alpha_{1} E_{k}}+\hat{\rho}_{k}^{\Phi}\left(S_{k}\right) E_{k}+\Phi_{k+1}\left(S_{k+1}\right)
\end{gathered}
$$

for all $k=1, \ldots, N$ and some $V_{0} \in \mathbb{R}$. This can be seen by substituting (5.15) and (5.17) into (5.8) 
Definition 5.4.1 In the model with cost of risk we call an arbitrage opportunity for a new trader a portfolio of European options $\Phi$ such that the value of its hedging portfolio of a new trader satisfies

$$
\begin{aligned}
& V_{0}=-\{\Phi\}_{\{\Psi\}, 0}^{s}\left(S_{0}\right) \\
& \mathbb{P}\left(V_{N} \geq 0\right)=1 \\
& \mathbb{P}\left(V_{N}>0\right)>0 .
\end{aligned}
$$

for some portfolio of options $\Psi$.

In other words an arbitrage opportunity for a new trader is a possibility for a trader holding the zero portfolio to buy options portfolio $\Phi$, hedge it optimally and at time $t_{N}$ get a riskless profit. We assume that all options in the market are available for their selling prices from various traders, holding their different portfolios. So the value $V_{0}=-\{\Phi\}_{\{\Psi\}, 0}^{s}\left(S_{0}\right)$ at time $t_{0}$ corresponds to the amount borrowed from the bank account $B$ for the purchase of portfolio $\Phi$ from a trader who holds portfolio $\Psi$. Note that by the definition (5.3) the price of an option portfolio is not included in the portfolio value before its expiry date. That is why the initial zero investment corresponds to minus the price of the portfolio.

The buying indifference prices (and also selling indifference prices in the presence of some non-zero portfolios) of positive payoffs may in principle turn out to be negative (see the example below). Nevertheless this does not necessarily mean that these payoffs are arbitrage opportunities, because the buyer of a risky option is obliged to put money on the reserve bank account according to the risk of his or her portfolio. Transferring this money from the regular to the reserve bank account makes the trader lose money.

In the classical definition of an arbitrage opportunity we gave in chapter 2 the trader always starts with an empty portfolio, so in the traditional formulation everyone is a 'new trader'. In this sense the definition of an arbitrage opportunity for a new trader is equivalent to the definition 2.2.3.

Example As a simple example we consider a one-step model with the following parameters: $\mu=0.05, r=0.03, \tilde{r}=0, \sigma=0.3, T=1, S_{0}=B_{0}=$ $1, \alpha_{1}=1, \alpha_{2}=20, \alpha_{3}=\alpha_{4}=0$. Then at time $t_{1}=T=1$

$$
\begin{aligned}
B_{1} & =1.0305, \\
S_{1} & =\left\{\begin{array}{ll}
1.3566 & \text { with probability } \frac{1}{2} \\
0.7445 & \text { with probability } \frac{1}{2}
\end{array} .\right.
\end{aligned}
$$

Consider a European Call $\Phi$ with strike $K=1$ and maturity $T_{1}=1$. Then its binomial price in this one-step model is

$$
\operatorname{Bin}_{0}^{1, \Phi}\left(S_{0}\right)=e^{-r T}\left(q_{0}^{u} \Phi\left(u_{0} S_{0}\right)+q_{0}^{d} \Phi\left(d_{0} S_{0}\right)\right)=0.1617
$$


and its Vega is

$$
\mathcal{V}_{0}^{1}=0.4116
$$

then

$$
\begin{aligned}
\hat{\rho}_{0}^{\Phi}\left(S_{0}\right) & =\alpha_{2}\left|\mathcal{V}_{0}\right|=8.2321, \\
E_{0,0} & =e^{-r}\left(e^{\tilde{r}}-e^{r}\right)=-0.0296,
\end{aligned}
$$

and hence by Theorem 5.3.1

$$
\{\Phi\}_{\{0\}, 0}^{b}\left(S_{0}\right)=\operatorname{Bin}_{0}^{1, \Phi_{1}}\left(S_{0}\right)+\hat{\rho}_{0}^{\Phi}\left(S_{0}\right) E_{0,0}=-0.0816 .
$$

The negative price we have got is explained by a very high value of parameter $\alpha_{2}$ and reflects the trader's fear of risk, which makes him or her very unwilling towards accepting this payoff. Under the same assumptions, by Lemma 5.3.3 the selling indifference price of $\Phi$ in the presence of $\Phi$ (which is the price the trader who holds $\Phi$ will ask for giving $\Phi$ away) is

$$
\begin{array}{r}
\{\Phi\}_{\{\Phi\}, 0}^{s}\left(S_{0}\right)=e^{-r T}\left(C_{0}^{\Phi}\left(S_{0}\right)-C_{0}^{\Phi-\Phi}\left(S_{0}\right)\right)=e^{-r T}\left(C_{0}^{\Phi}\left(S_{0}\right)-C_{0}^{0}\left(S_{0}\right)\right) \\
=\{\Phi\}_{\{0\}, 0}^{b}\left(S_{0}\right)
\end{array}
$$

the price a trader without a portfolio will offer for having $\Phi$. And thus

$$
\{\Phi\}_{\{\Phi\}, 0}^{s}\left(S_{0}\right)=-0.0816 .
$$

Again, the punishment for the risk is so high $\left(\alpha_{2}=20\right)$ that the holder of $\Phi$ is ready to get rid of it even for a negative price.

In lemma 5.4.1 and theorem 5.4.2 we prove that our model does not allow arbitrage opportunities for a new trader.

Lemma 5.4.1 If $V$ is the value of a hedging portfolio for options portfolio $\Phi$ of a new trader with

$$
V_{0}=-\{\Phi\}_{\{\Psi\}, 0}^{s}\left(S_{0}\right),
$$

for some options portfolio $\Psi$ then $V_{N}$ satisfies the following inequality

$$
\begin{aligned}
V_{N}(\omega) \leq & \sum_{k=0}^{N-1}\left(\hat{\rho}_{k}^{\Phi}\left(S_{k}(\omega)\right)-\boldsymbol{E}\left[\hat{\rho}_{k}^{\Phi}\left(S_{k}\right)\right]\right) E_{N, k} \\
& +\sum_{k=0}^{N-1} \frac{\left(\boldsymbol{E}_{k}\left[R_{k}\right]-e^{r \Delta t_{k}}\right)\left(\boldsymbol{E}_{k}\left[R_{k}\right]-R_{k}(\omega)\right)}{2 \alpha_{1} E_{k}} e^{r\left(t_{N}-t_{k+1}\right)}
\end{aligned}
$$

for all $\omega \in \Omega$. 
Proof By induction in $k$ we prove that for all $k=0, \ldots, N$

$$
\begin{aligned}
V_{k} \leq- & \sum_{i=k+1}^{N} \operatorname{Bin}_{k}^{i, \Phi_{i}}\left(S_{k}\right)-\sum_{i=0}^{N-1} \mathbf{E}\left[\hat{\rho}_{i}^{\Phi}\left(S_{i}\right)\right] E_{k, i}+\sum_{i=0}^{k-1} \hat{\rho}_{i}^{\Phi}\left(S_{i}\right) E_{k, i} \\
& +\sum_{i=0}^{k-1} \frac{\left(\mathbf{E}_{i}\left[R_{i}\right]-e^{r \Delta t_{i}}\right)\left(\mathbf{E}_{i}\left[R_{i}\right]-R_{i}\right)}{2 \alpha_{1} E_{i}} e^{r\left(t_{k}-t_{i+1}\right)}
\end{aligned}
$$

1. $k=0$.

$$
V_{0}=-\{\Phi\}_{\{\Psi\}, 0}^{s}\left(S_{0}\right)
$$

then by theorem 5.3 .5

$$
V_{0} \leq-\{\Phi\}_{\{0\}, 0}^{b}\left(S_{0}\right)
$$

therefore by theorem 5.3.1

$$
V_{0} \leq-\sum_{i=1}^{N} \operatorname{Bin}_{0}^{i, \Phi_{i}}\left(S_{0}\right)-\sum_{i=0}^{N-1} \mathbf{E}\left[\hat{\rho}_{i}^{\Phi}\left(S_{i}\right)\right] E_{0, i} .
$$

2. Assume the statement is proved for $0, \ldots, k$. By (5.28) we write

$$
\begin{gathered}
V_{k+1}=V_{k} e^{r \Delta t}-S_{k} \hat{\Delta}_{k}^{\Phi}\left(R_{k}-e^{r \Delta t_{k}}\right)-\frac{\left(R_{k}-e^{r \Delta t_{k}}\right)\left(\mathbf{E}_{k}\left[R_{k}\right]-e^{r \Delta t_{k}}\right)}{2 \alpha_{1} E_{k}} \\
+\frac{\left(\mathbf{E}_{k}\left[R_{k}\right]-e^{r \Delta t_{k}}\right)^{2}}{2 \alpha_{1} E_{k}}+\hat{\rho}_{k}^{\Phi}\left(S_{k}\right) E_{k}+\Phi_{k+1}\left(S_{k+1}\right) .
\end{gathered}
$$

By the induction assumption

$$
\begin{aligned}
V_{k+1} \leq( & -\sum_{i=k+1}^{N} \operatorname{Bin}_{k}^{i, \Phi_{i}}\left(S_{k}\right)-\sum_{i=0}^{N-1} \mathbf{E}\left[\hat{\rho}_{i}^{\Phi}\left(S_{i}\right)\right] E_{k, i}+\sum_{i=0}^{k-1} \hat{\rho}_{i}^{\Phi}\left(S_{i}\right) E_{k, i} \\
& \left.+\sum_{i=0}^{k-1} \frac{\left(\mathbf{E}_{i}\left[R_{i}\right]-e^{r \Delta t_{i}}\right)\left(\mathbf{E}_{i}\left[R_{i}\right]-R_{i}\right)}{2 \alpha_{1} E_{i}} e^{r\left(t_{k}-t_{i+1}\right)}\right) e^{r \Delta t_{k}} \\
& -S_{k} \hat{\Delta}_{k}^{\Phi}\left(R_{k}-e^{r \Delta t_{k}}\right)-\frac{\left(R_{k}-e^{r \Delta t_{k}}\right)\left(\mathbf{E}_{k}\left[R_{k}\right]-e^{r \Delta t_{k}}\right)}{2 \alpha_{1} E_{k}} \\
& +\frac{\left(\mathbf{E}_{k}\left[R_{k}\right]-e^{r \Delta t_{k}}\right)^{2}}{2 \alpha_{1} E_{k}}+\hat{\rho}_{k}^{\Phi}\left(S_{k}\right) E_{k}+\Phi_{k+1}\left(S_{k+1}\right) .
\end{aligned}
$$


Since $\hat{\Delta}_{k}^{\Phi}=\sum_{i=k+1}^{N} \hat{\Delta}_{k}^{i, \Phi_{i}}$ is the sum of binomial Deltas of payoffs to be paid after time $t_{k}$, the following is true

$$
\sum_{i=k+1}^{N} e^{r \Delta t_{k}} \operatorname{Bin}_{k}^{i, \Phi_{i}}\left(S_{k}\right)+S_{k} \hat{\Delta}_{k}^{\Phi}\left(R_{k}-e^{r \Delta t_{k}}\right)=\sum_{i=k+1}^{N} \operatorname{Bin}_{k+1}^{i, \Phi_{i}}\left(S_{k+1}\right) .
$$

Note that by the definition of the binomial price

$$
\operatorname{Bin}_{k}^{k, \Phi_{k}}\left(S_{k}\right)=\Phi_{k}\left(S_{k}\right)
$$

for all $k=1, \ldots, N$. We should also mention that

$$
\begin{aligned}
E_{k, i} e^{r \Delta t_{k}} & =e^{\tilde{r} t_{i}-r\left(t_{i+1}-t_{k+1}\right)}\left(e^{\tilde{r} \Delta t_{i}}-e^{r \Delta t_{i}}\right)=E_{k+1, i}, \\
E_{k} & =e^{\tilde{r} t_{k}-r\left(t_{k+1}-t_{k+1}\right)}\left(e^{\tilde{r} \Delta t_{k}}-e^{r \Delta t_{k}}\right)=E_{k+1, k} .
\end{aligned}
$$

Applying (5.31)-(5.34) to (5.30) we get

$$
\begin{aligned}
V_{k+1} \leq- & \sum_{i=k+2}^{N} \operatorname{Bin}_{k+1}^{i, \Phi_{i}}\left(S_{k+1}\right)-\sum_{i=0}^{N-1} \mathbf{E}\left[\hat{\rho}_{i}^{\Phi}\left(S_{i}\right)\right] E_{k+1, i}+\sum_{i=0}^{k} \hat{\rho}_{i}^{\Phi}\left(S_{i}\right) E_{k+1, i} \\
& +\sum_{i=0}^{k} \frac{\left(\mathbf{E}_{i}\left[R_{i}\right]-e^{r \Delta t_{i}}\right)\left(\mathbf{E}_{i}\left[R_{i}\right]-R_{i}\right)}{2 \alpha_{1} E_{i}} e^{r\left(t_{k+1}-t_{i+1}\right)},
\end{aligned}
$$

so (5.29) is true for all $k=0, \ldots, N$. Substituting $k=N$ gives us the statement of the lemma:

$$
\begin{aligned}
V_{N} \leq & \sum_{i=0}^{N-1}\left(\hat{\rho}_{i}^{\Phi}\left(S_{i}\right)-\mathbf{E}\left[\hat{\rho}_{i}^{\Phi}\left(S_{i}\right)\right]\right) E_{N, i} \\
& +\sum_{i=0}^{N-1} \frac{\left(\mathbf{E}_{i}\left[R_{i}\right]-e^{r \Delta t_{i}}\right)\left(\mathbf{E}_{i}\left[R_{i}\right]-R_{i}\right)}{2 \alpha_{1} E_{i}} e^{r\left(t_{N}-t_{i+1}\right)}
\end{aligned}
$$

As a corollary of Lemma 5.4.1 we prove that our model admits no arbitrage opportunities for new traders.

Theorem 5.4.2 (No Arbitrage) There are no portfolios of options $\Phi$ and $\Psi$ such that the value of the hedging portfolio for $\Phi$ of a new trader satisfies

$$
\begin{aligned}
& V_{0}=-\{\Phi\}_{\{\Psi\}, 0}^{s}\left(S_{0}\right) \\
& \mathbb{P}\left(V_{N} \geq 0\right)=1 \\
& \mathbb{P}\left(V_{N}>0\right)>0 .
\end{aligned}
$$


Proof By Lemma 5.4.1 from (5.35) the value of the hedging portfolio for $\Phi$ of a new trader satisfies

$$
\begin{aligned}
V_{N} \leq & \sum_{k=0}^{N-1}\left(\hat{\rho}_{k}^{\Phi}\left(S_{k}\right)-\mathbf{E}\left[\hat{\rho}_{k}^{\Phi}\left(S_{k}\right)\right]\right) E_{N, k} \\
& \quad+\sum_{k=0}^{N-1} \frac{\left(\mathbf{E}_{k}\left[R_{k}\right]-e^{r \Delta t_{k}}\right)\left(\mathbf{E}_{k}\left[R_{k}\right]-R_{k}\right)}{2 \alpha_{1} E_{k}} e^{r\left(t_{N}-t_{k+1}\right)}
\end{aligned}
$$

Since the expectation of the random variable on the right is zero, then taking expectations on both sides gives us

$$
\mathbf{E}\left[V_{N}\right] \leq 0
$$

which contradicts (5.36) and (5.37). Therefore there is no arbitrage for a new trader in the model with cost of risk. 


\section{Chapter 6}

\section{Numerical Results}

This chapter presents the numerical results we have obtained from the model with cost of risk. First we show the buying and the selling indifference prices for some values of parameters and some portfolios of European options to demonstrate the key properties of the model. Then we present the calibration results of the indifference mid prices to the market prices.

\subsection{Indifference Prices}

To demonstrate the main properties of the indifference prices of the model with cost of risk we present the buying and the selling indifference prices of at the money Calls and Puts in the presence of different portfolios for a given (artificial) set of model parameters. We also compare these prices to the prices of the same options in a binomial model with the corresponding market parameters. The following values of the model parameters are used:

$$
\begin{array}{cl}
\mu=0.05 & \sigma=0.3 \\
r=0.03 & \tilde{r}=0.0 \\
S_{0}=1.0 & T=1.0 \\
\alpha_{1}=1.0 . &
\end{array}
$$

For simplicity we use $\alpha_{3}=\alpha_{4}=0$. All observations hold true for other values of $\alpha_{3}$ and $\alpha_{4}$ and are simple corollaries of the pricing theorem 5.3.1. We take the Put and the Call with the same maturities $T_{\text {Put }}=T_{\text {Call }}=1.0$ year and strikes $K_{\mathrm{Put}}=K_{\mathrm{Call}}=1.0$. The results are presented in table 6.1 .

First of all we note that the Put-Call parity price $\{\text { Call }-\mathrm{Put}\}_{0}^{s}$ does not depend on the value of $\alpha$ and is equal to its theoretical value $S_{0}-K e^{-r T}=$ 0.0296. This should not be a surprise, since all sensitivities apart from Delta 


\begin{tabular}{|c|c|c|c|c|c|}
\hline option, $\alpha_{2} \rightarrow$ & 0.01 & 0.1 & 0.5 & 1 & BS Price \\
\hline$\{\mathrm{Put}\}_{0}^{s}$ & 0.1033 & 0.1035 & 0.1041 & 0.1049 & 0.1033 \\
\hline$\{\text { Put }\}_{0}^{b}$ & 0.1033 & 0.1031 & 0.1025 & 0.1017 & 0.1033 \\
\hline$\{\mathrm{Put}\}_{\{-\mathrm{Put}\}}^{s}$ & 0.1034 & 0.1037 & 0.1057 & 0.1081 & 0.1033 \\
\hline$\{\text { Call }\}_{0}^{s}$ & 0.1329 & 0.1330 & 0.1337 & 0.1345 & 0.1328 \\
\hline$\{\text { Call }\}_{0}^{b}$ & 0.1328 & 0.1327 & 0.1320 & 0.1312 & 0.1328 \\
\hline$\{\text { Call }\}_{\{- \text {Call }\}}^{s}$ & 0.1329 & 0.1333 & 0.1352 & 0.1377 & 0.1328 \\
\hline$\{\text { Call }- \text { Put }\}_{0}^{s}$ & 0.0296 & 0.0296 & 0.0296 & 0.0296 & 0.0296 \\
\hline$\{\text { Put }\}^{s}$ & 0.1032 & 0.1031 & 0.1024 & 0.1016 & 0.1033 \\
\hline Call $\}_{\text {-Put }}^{b}$ & 0.1329 & 0.1331 & 0.1337 & 0.1345 & 0.1328 \\
\hline
\end{tabular}

Table 6.1: Indifference Prices

of this portfolio are zero and therefore the portfolio can be hedged in a riskless way so there is no change in the price. The more formal argument is based on Theorem 5.3.1: if all Greeks are zero apart from Delta, then $\hat{\rho}_{k}^{\text {Call-Put }}(S)=0$ for all $k=0, \ldots, N-1$ and all $S \in \mathbb{R}$, so the indifference price of the portfolio is equal to the binomial price, which satisfies the CallPut parity.

If we look at the indifference prices of the Put and the Call in the presence of the zero portfolio, then we see that the selling prices $\{\mathrm{Put}\}_{0}^{s}$ and $\{\mathrm{Call}\}_{0}^{s}$ are higher and the buying prices $\{\mathrm{Put}\}_{0}^{b}$ and $\{\mathrm{Call}\}_{0}^{b}$ are lower than the binomial prices. In other words, the trader wants to receive more for selling an option and to pay less for buying it — the model produces bid-ask spreads. The risk of a portfolio consisting of options is always bigger than zero. Therefore if a trader holding the zero portfolio buys an option he or she is obliged to put a certain positive amount of money in the reserve bank account, which earns a lower interest rate. So he or she wishes to be compensated for this loss by receiving a higher premium. Note, that the bid-ask spreads widen if $\alpha_{2}$, the cost of the Vega risk, grows. The selling prices of a Call or a Put in the presence of a short position in the same option grow even faster with the growth of $\alpha_{2}$ than the same prices in the presence of the zero portfolio (compare $\{\mathrm{Put}\}_{\{-\mathrm{Put}\}}^{s}$ and $\{\mathrm{Put}\}_{0}^{s},\{\mathrm{Call}\}_{\{-\mathrm{Call}\}}^{s}$ and $\left.\{\mathrm{Call}\}_{0}^{s}\right)$ since here selling adds an extra risk to an already risky portfolio.

Finally we would like to draw the reader's attention to the fact that the ask prices of the Put become lower and bid prices of the Call become higher in the presence of their Put-Call parity counterparts: $\{\mathrm{Put}\}_{\{\mathrm{Call}\}}^{s}$ ' 
$\{\text { Call }\}_{\{- \text {Put }\}}^{b}$. This is exactly what we should expect. Since Put and Call positions with opposite signs reduce each other's hedging risk, a trader is ready to pay more to get one of them to his or her portfolio, if he or she already has the other, and the trader asks for less money, when he or she is selling them.

\subsection{Calibration to the Market Data}

\subsubsection{Parameters' Interpretation}

We have calibrated the mid indifference prices of the model with cost of risk to the market prices of a few European options. As we have already mentioned in section 5.1.2, $\alpha_{1}$ is the risk aversion parameter and influences only the hedging strategies, not the price, while $\alpha_{2}$ is responsible for bid-ask spreads and does not influence the mid prices in the presence of the zero portfolio. Other parameters of the risk function $\alpha_{3}$ and $\alpha_{4}$ and also the volatility $\sigma$ of the underlying model can be used for the calibration of the indifference mid prices. We used a fixed value for the drift parameter $\mu$.

Consider a one year Call. By Theorem 5.3.1 its indifference mid price in the presence of the zero portfolio at time $t_{0}$ is

$$
\begin{aligned}
\left\{\mathrm{Call}_{\{0\}, 0}^{m}\left(S_{0}\right)=\right. & \frac{\{\Phi\}_{\{0\}, 0}^{s}\left(S_{0}\right)+\{\Phi\}_{\{0\}, 0}^{b}\left(S_{0}\right)}{2} \\
= & \operatorname{Bin}_{0}^{\Phi}\left(S_{0}\right)+\sum_{i=0}^{N-1} \mathbf{E}_{0}\left[\frac{\hat{\rho}_{i}^{\Phi}\left(S_{i}\right)-\hat{\rho}_{i}^{\Phi}\left(S_{i}\right)}{2}\right] E_{0, i} \\
= & \operatorname{Bin}_{0}^{\Phi}\left(S_{0}\right) \\
& +\sum_{i=0}^{N-1} \mathbf{E}_{0}\left[\frac{\max \left(\alpha_{3} \operatorname{vanna}_{i}^{\Phi}\left(S_{i}\right), 0\right)-\max \left(-\alpha_{3} \operatorname{vanna}_{i}^{\Phi}\left(S_{i}\right), 0\right)}{2}\right] E_{0, i} \\
& +\sum_{i=0}^{N-1} \mathbf{E}_{0}\left[\frac{\max \left(-\alpha_{4} \operatorname{volga}_{i}^{\Phi}\left(S_{i}\right), 0\right)-\max \left(\alpha_{4} \operatorname{volga}_{i}^{\Phi}\left(S_{i}\right), 0\right)}{2}\right] E_{0, i} \\
= & \operatorname{Bin}_{0}^{\Phi}\left(S_{0}\right)+\frac{1}{2} \sum_{i=0}^{N-1} \mathbf{E}_{0}\left[\alpha_{3} \operatorname{vanna}_{i}^{\Phi}\left(S_{i}\right)-\alpha_{4} \operatorname{volga}_{i}^{\Phi}\left(S_{i}\right)\right] E_{0, i} .
\end{aligned}
$$

If we now look at the graphs of Vanna and Volga presented in figures 6.1 and 6.2 of a European Call option with maturity 1 year we see that the correction to the theoretical binomial price depends on the strike. Note, that $E_{0, i}<0$ for all $i=0, \ldots, N-1$ hence the Volga correction is positive and is higher 


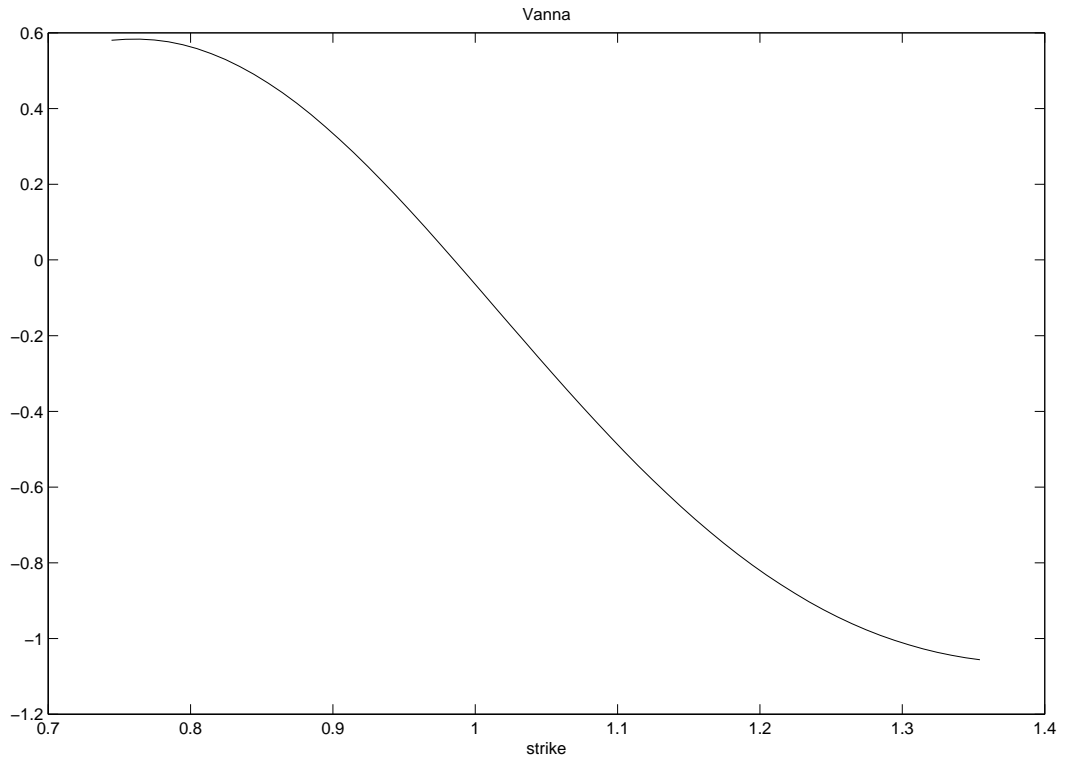

Figure 6.1: Vanna of a 1 year option, $r=0.03, \sigma=0.3$.

for the far in the money and far out of the money options than for at the money options. The Vanna term changes sign from plus to minus (or from minus to plus - depending on the sign of $\alpha_{3}$ ) and therefore makes one side of strikes more and another less expensive than their binomial prices. In other words these correction terms create a smile and while Volga regulates the curvature, Vanna affects the skew. The parameter $\sigma$ affects the level of the at the money volatility.

The graphs in figures 6.3 and 6.4 show various smiles that can be obtained by changing one of parameters $\alpha_{3}$ and $\alpha_{4}$ and setting the other one to be zero. If both parameters are zero, then the smile is almost flat, because the option prices are equal to the binomial prices. Changes in $\alpha_{3}$ affect the slope of the smile, while the changes in $\alpha_{4}$ affect the smile's curvature. We would like to point out that we consider both positive and negative values of $\alpha_{3}$. Due to the negative correlation between stock prices and their volatilities the smiles of stock markets are skewed in favor of low strikes. This corresponds to positive values of the parameter $\alpha_{3}$. Nevertheless foreign exchange rates may have either positive or negative correlations with their volatilities and therefore their smiles may be skewed in favor of either low or of high strikes, corresponding to either positive or negative values of $\alpha_{3}$. 


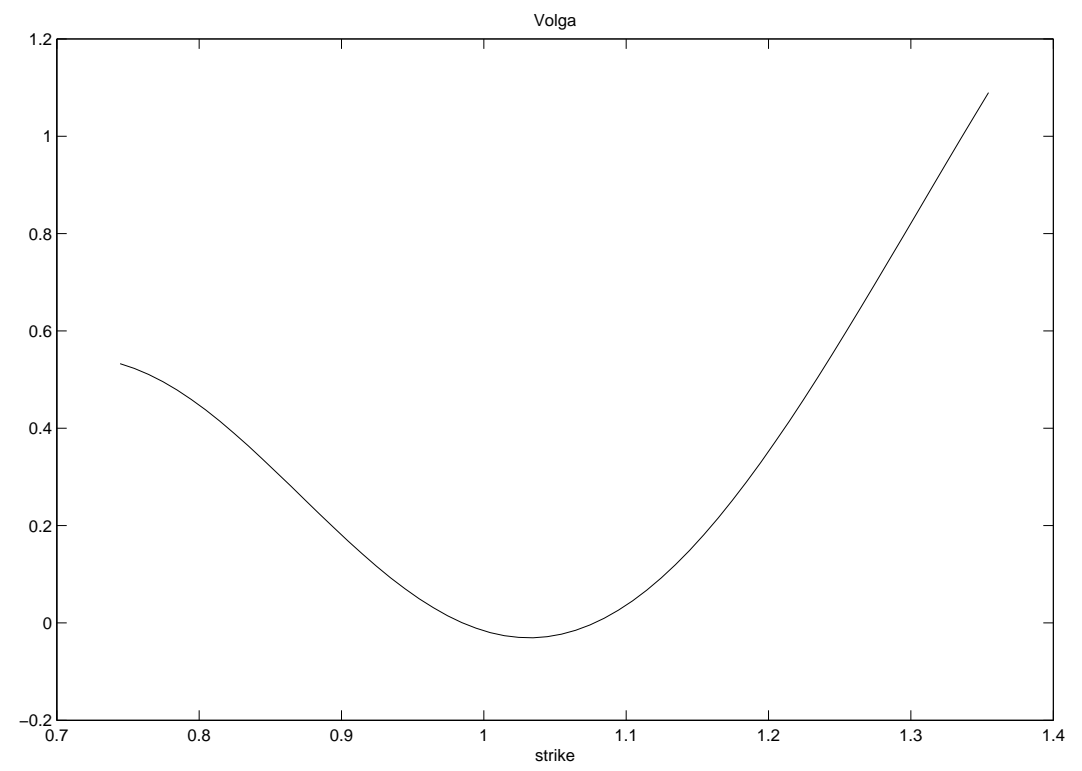

Figure 6.2: Volga of a 1 year option, $r=0.03, \sigma=0.3$.

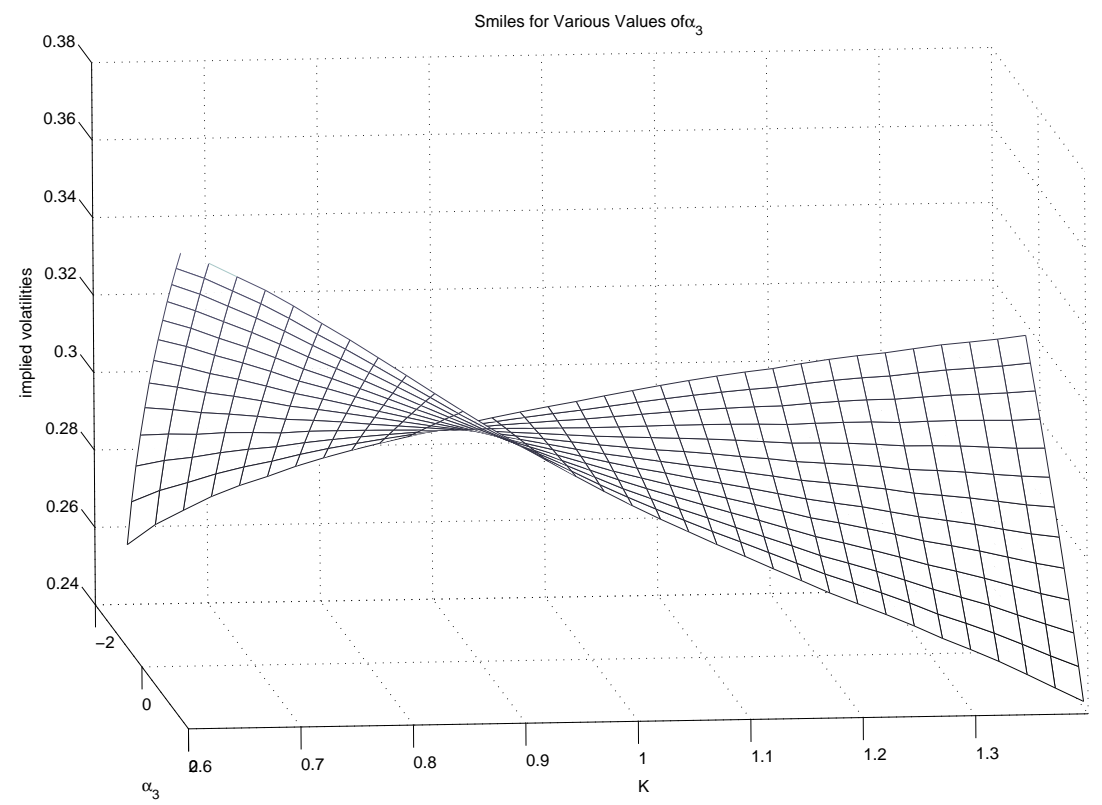

Figure 6.3: Volatility Smiles Against $\alpha_{3},\left(\alpha_{4}=0\right)$. 


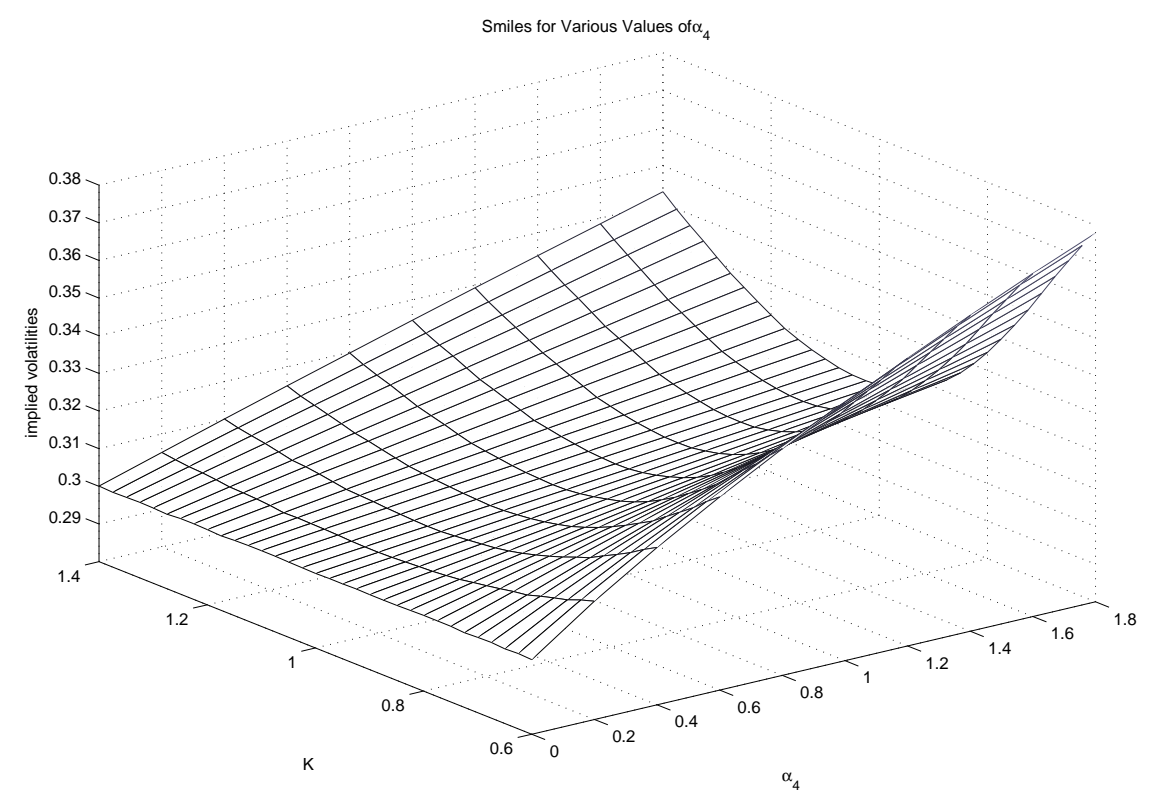

Figure 6.4: Volatility Smiles Against $\alpha_{4},\left(\alpha_{3}=0\right)$.

\subsubsection{Calibration Algorithm}

There are three model parameters to be calibrated: $\sigma, \alpha_{3}$ and $\alpha_{4}$. Our model has the same drawback as many other market models: it is impossible to fit its parameters to the whole implied volatility surface at once. Fitted parameters are different for different expiration dates especially for very short and very long maturities. Maybe a way to overcome this problem is the introduction of a term structure on the calibration parameters, which might make the model more realistic but will substantially increase the number of parameters. We run the calibration procedure for each maturity independently, hence we find a set of parameters for all options with various strikes and the same maturity date. A different set of parameters corresponds to each maturity date.

A straightforward three-dimensional calibration is extremely time consuming: each new value of volatility $\sigma$ requires recalculation of Vannas and Volgas for all nodes of the binomial tree (5.2). To avoid this, we perform the minimization in steps. Each step involves calibration of $\alpha_{3}$ and $\alpha_{4}$ for a fixed value of the volatility parameter $\sigma$. The parameter $\sigma$ changes from step to step in order to fit the at the money implied volatility.

For each maturity the model is fitted using three market option prices an at the money Call option $\Phi_{1}$, an in the money Call option $\Phi_{2}$ and an out 
of the money Call option $\Phi_{3}$. If we denote their market prices respectively by $\Phi_{1}^{\text {market }}, \Phi_{2}^{\text {market }}$, and $\Phi_{3}^{\text {market }}$ the calibration algorithm is described by the following equations:

$$
\begin{aligned}
& \left(\alpha_{3}^{i}\left(\sigma^{i}\right), \alpha_{4}^{i}\left(\sigma^{i}\right)\right)=\arg \min _{\alpha_{3}^{i}, \alpha_{4}^{i}}\left\{\operatorname{Err}\left(\sigma^{i}, \alpha_{3}^{i}, \alpha_{4}^{i}\right)\right\}, \quad i=0,1, \ldots \\
& \operatorname{Err}\left(\sigma, \alpha_{3}, \alpha_{4}\right)=\sum_{j=1}^{3}\left|\Phi_{j}^{\text {market }}-\left\{\Phi_{j}\right\}_{\{0\}, 0}^{m}\left(\sigma^{i}, \alpha_{3}^{i}, \alpha_{4}^{i}\right)\right| \\
& \sigma^{i}=\sigma^{i-1}-\sigma_{\Delta}^{i-1}, \quad i=1,2, \ldots \\
& \sigma_{\Delta}^{i}=\hat{\sigma}\left(\left\{\Phi_{1}\right\}_{\{0\}, 0}^{m}\left(\sigma^{i}, \alpha_{3}^{i}\left(\sigma^{i}\right), \alpha_{4}^{i}\left(\sigma^{i}\right)\right)\right)-\hat{\sigma}\left(\Phi_{1}^{\text {market }}\right), \\
& \sigma^{0}=\hat{\sigma}\left(\Phi_{1}^{\text {market }}\right) \\
& i=0,1, \ldots \text {. }
\end{aligned}
$$

where $\hat{\sigma}(P)$ denotes the implied volatility corresponding to the option price $P$. In other words, we begin with $\sigma$ equal to the at the money implied volatility and then on each step minimize the error function by changing parameters $\alpha_{3}$ and $\alpha_{4}$. Changing $\alpha_{3}$ and $\alpha_{4}$ changes the at the money volatility of the model smile. So after choosing the values for these parameters we change the value of $\sigma$ in such a way that the at the money volatility of the model is equal to the at the money volatility of the market. We follow this algorithm until the fitting error $\operatorname{Err}\left(\sigma^{i}, \alpha_{3}^{i}, \alpha_{4}^{i}\right)$ is smaller than a certain tolerance parameter $\epsilon$ :

$$
\operatorname{Err}\left(\sigma^{i}, \alpha_{3}^{i}, \alpha_{4}^{i}\right)>\epsilon
$$

This algorithm has two drawbacks. First, its results depend on the choice of in the money and out of the money options. As an example let us consider the historical prices on the 6th of February 2006 for European Call options on the German stock index DAX expiring on the 16th of June 2006. The closing price of DAX on that date was 5652.11. We calibrate the model twice. First to the options with strikes 5050, 5700 and 6400 (which approximately corresponds to Deltas equal to $0.9,0.5$ and 0.1 respectively) and then with strikes 5350, 5700 and 6100 (corresponding Deltas are approximately 0.75, 0.5 and 0.25 ). The fitted parameters are presented in table 6.2. One may see that while the values of $\sigma$ and $\alpha_{3}$ differ only moderately, the values of $\alpha_{4}$ differ by more than $25 \%$. Nevertheless if we look at the smile fits in figure 6.5 we see that both parameter sets give a reasonably good fit. The first set performs better for strikes far in and out of the money and the second performs better for strikes closer to the spot price. Therefore the calibration sets of options should be chosen carefully depending on the situation. 


$\begin{array}{ccc}\text { parameter } & \text { options set } 1 & \text { options set } 2 \\ \sigma & 0.1584 & 0.1595 \\ \alpha_{3} & 0.9469 & 0.9823 \\ \alpha_{4} & 0.7755 & 0.5534\end{array}$

Table 6.2: Fitted Parameters for DAX Jun 2006, Feb 6, 2006

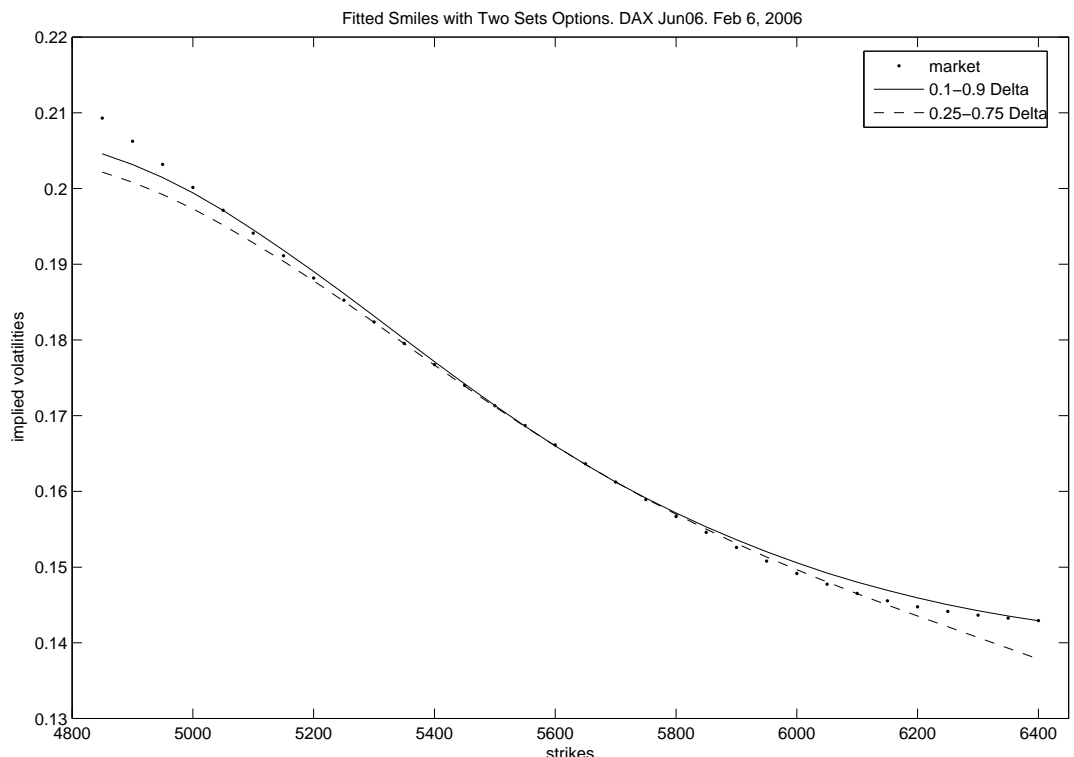

Figure 6.5: Fitted Smiles for Two Sets of Options. 


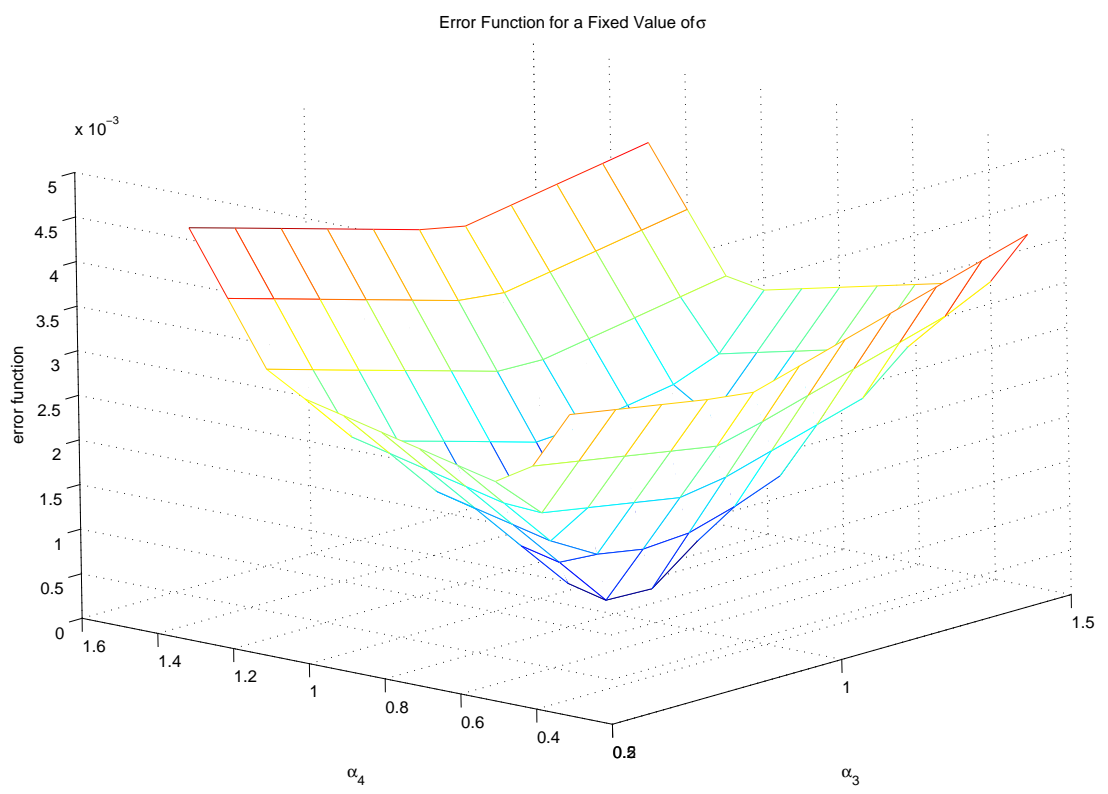

Figure 6.6: The Error Function for a Fixed $\sigma$. DAX Jun06, Feb 6, 2006.

As a second drawback we should mention the fact that the is no guarantee that the result of the algorithm is the global 3-dimensional minimum. Nevertheless, the empirical experience with our algorithm suggests that for a fixed $\sigma=\bar{\sigma}$ the function $\operatorname{Err}\left(\bar{\sigma}, \alpha_{3}, \alpha_{4}\right)$ has no other local minima in the neighborhood of the solution: see figure 6.6 for the graph of the fitting error of Jun06 DAX options for $\sigma=0.1584$. We got similar pictures for other options and other values of the volatility parameter. Moreover, the algorithm converges for all smiles we have experimented with. With a faster computer one could use the result of our fitting procedure as the initial point for a full three-dimensional minimization.

\subsubsection{Calibration Results}

For the calibration of the model we used the data for a few dates in February 2006 for DAX European options kindly provided by Saen Options BV. We have chosen DAX because its price is defined on the basis of reinvested dividends, or in other words, its price dynamics do not have discrete dividends. The historical average growth rate for DAX changed from negative to very positive in the past 5 years. We decided to fix the value of the drift parameter $\mu$ at $5 \%$ because both interest rates and volatilities were very low in the beginning of 2006. The values of the calibrated parameters and the 


$\begin{array}{rllllll}\text { date } & \sigma & \alpha_{3} & \alpha_{4} & \begin{array}{l}\text { av. vol } \\ \text { error }\end{array} & \begin{array}{l}\text { av. vol } \\ \text { error } \\ 25-75 \Delta\end{array} \\ 06.2 .2006 & 0.2353 & 1.7605 & 0.4046 & 0.000082 & 0.00030 \\ 13.2 .2006 & 0.2419 & 1.9198 & 0.3264 & 0.000080 & 0.00021 \\ 20.2 .2006 & 0.2391 & 1.9234 & 0.2944 & 0.000081 & 0.00027 \\ 27.2 .2006 & 0.2365 & 1.7820 & 0.3418 & 0.000059 & 0.00025\end{array}$

Table 6.3: Calibration Results for DAX June 2007 option on various dates

fitting errors can be found in Tables 6.3, 6.4 and 6.5. One can see that the values of the parameters are pretty stable and qualitatively follow our expectations. If we compare figure 6.8 with table 6.4 , figure 6.7 with table 6.3 and figure 6.9 with table 6.5 we see that flatter smiles have lower values of the risk function parameters $\alpha_{3}$ and $\alpha_{4}$ and that lower smiles have lower values of $\sigma$. Note that parameter $\alpha_{4}$ in table 6.4 increases as the time to maturity becomes smaller. This increase reflects the fact that the curvature of implied volatility smiles is higher for shorter maturities.

Error function in tables 6.3 and 6.5 is the value $\operatorname{Err}\left(\sigma, \alpha_{3}, \alpha_{4}\right)$ for the set of calibrated parameters, e.g. the sum of the errors in prices for the three chosen options. Average volatility error is the average absolute difference between market and calibrated implied volatilities for all options considered, (not just three). For shorter maturities (see options expiring in March, April, June and September 2006) we compare average volatility errors calculated over strikes corresponding to Deltas 0.05 to 0.95 and 0.25 to 0.75 , see the last two columns in tables 6.5 and 6.4. For longer maturities (see options expiring in June 2007 in table 6.3) data for strikes corresponding to Deltas below 0.4 was not available. For those options we took the average over all available strikes (approximately from 0.8 to 0.4 Delta). Usually the volatility bid-ask spread for DAX options is 0.001-0.002. So all our fits are always inside the bid-ask spread boundaries and are well inside the boundaries for strikes closer to the spot price and for options with longer maturities.

Figures 6.10-6.20 present the fitted smiles - the implied volatilities of the prices produced with the fitted parameters - plotted against the market implied volatilities. Overall we have managed to get very accurate fits, especially for longer maturities. Shorter maturities demonstrate a slight discrepancy at the far ends of the range of strikes. Note that almost everywhere our model underestimates volatilities for small strikes due to the shape of Vannas and Volgas in that area (see figures 6.1 and 6.2). We would like to stress that those values of strikes correspond to values of Delta less that 0.1 , 


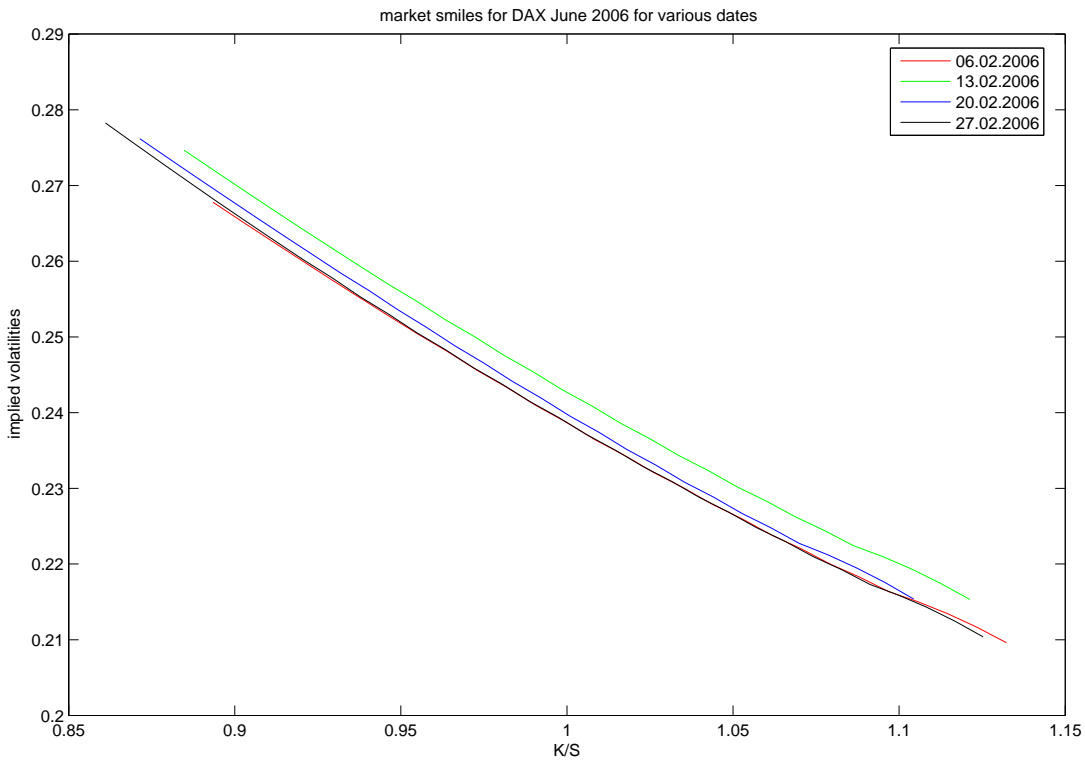

Figure 6.7: Market Volatility Smiles for for DAX June 2007 on various dates

$\begin{array}{rlllllll}\text { expiration } & & & & & \begin{array}{l}\text { av. vol } \\ \text { error } \\ \text { function }\end{array} & \begin{array}{l}\text { av. vol } \\ \text { error } 95 \Delta\end{array} & \begin{array}{l}\text { error } \\ 25-75 \Delta\end{array} \\ 17.3 .2006 & 0.1569 & 1.3954 & 2.8020 & 0.000002 & 0.00098 & 0.00081 \\ 21.4 .2006 & 0.1542 & 1.0256 & 1.2986 & 0.000001 & 0.00072 & 0.00048 \\ 16.6 .2006 & 0.1584 & 0.9469 & 0.7755 & 0.000002 & 0.00087 & 0.00060 \\ 15.9 .2006 & 0.1625 & 0.8836 & 0.3794 & 0.000011 & 0.00091 & 0.00037\end{array}$

Table 6.4: Calibration Results for DAX of various expirations on the 6.2.2006

\begin{tabular}{|c|c|c|c|c|c|c|}
\hline date & $\sigma$ & $\alpha_{3}$ & $\alpha_{4}$ & $\begin{array}{l}\text { error } \\
\text { function }\end{array}$ & $\begin{array}{l}\text { av. vol } \\
\text { error } \\
5-95 \Delta\end{array}$ & $\begin{array}{l}\text { av. vol } \\
\text { error } \\
25-75 \Delta\end{array}$ \\
\hline 006 & 0.1569 & 1.3954 & 2.8020 & 0.00 & 0.00098 & 0.00081 \\
\hline 13.2 .2006 & 0.1579 & 1.5116 & 3.5816 & 0.000001 & 0.00042 & 0.00015 \\
\hline 0.2 .2006 & 0.1457 & 1.7144 & 4.4663 & 0.000001 & 0.00090 & 0.00064 \\
\hline .2 .2006 & 0.1430 & 1.5437 & 4.8973 & 0.000065 & 0.00012 & 0.00008 \\
\hline
\end{tabular}

Table 6.5: Calibration Results for DAX March 2006 option on various dates 


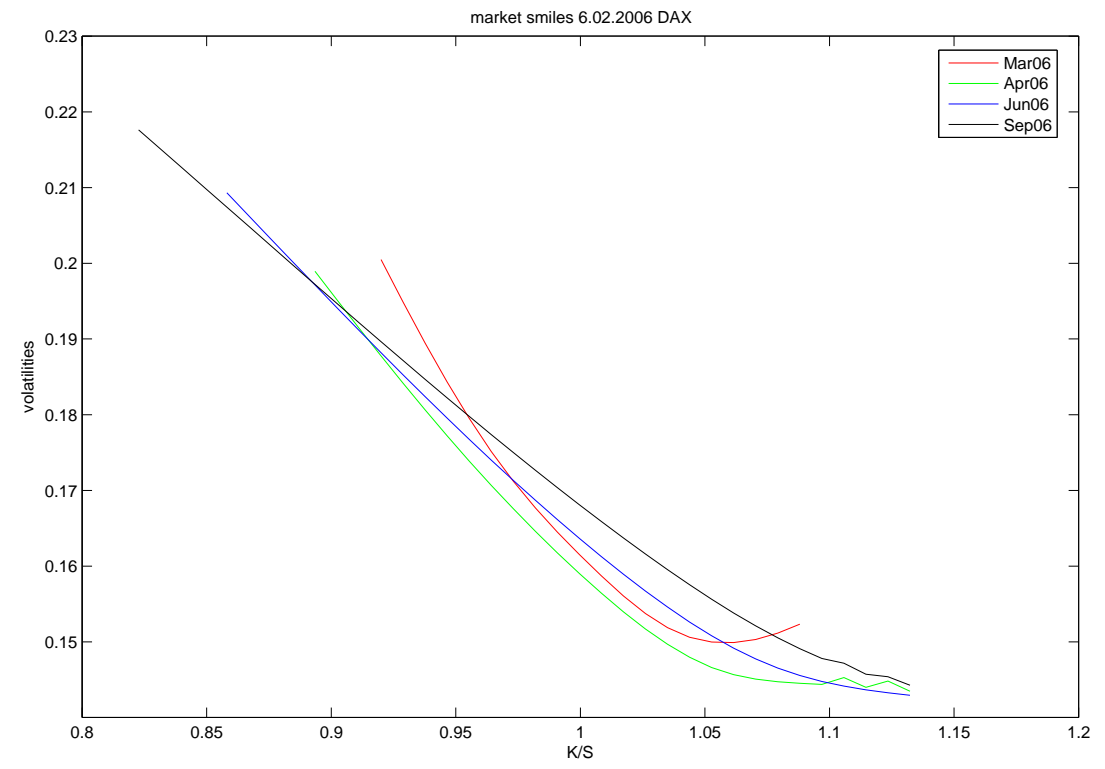

Figure 6.8: DAX Volatility Smiles for Various Maturities on the 6.2.2006

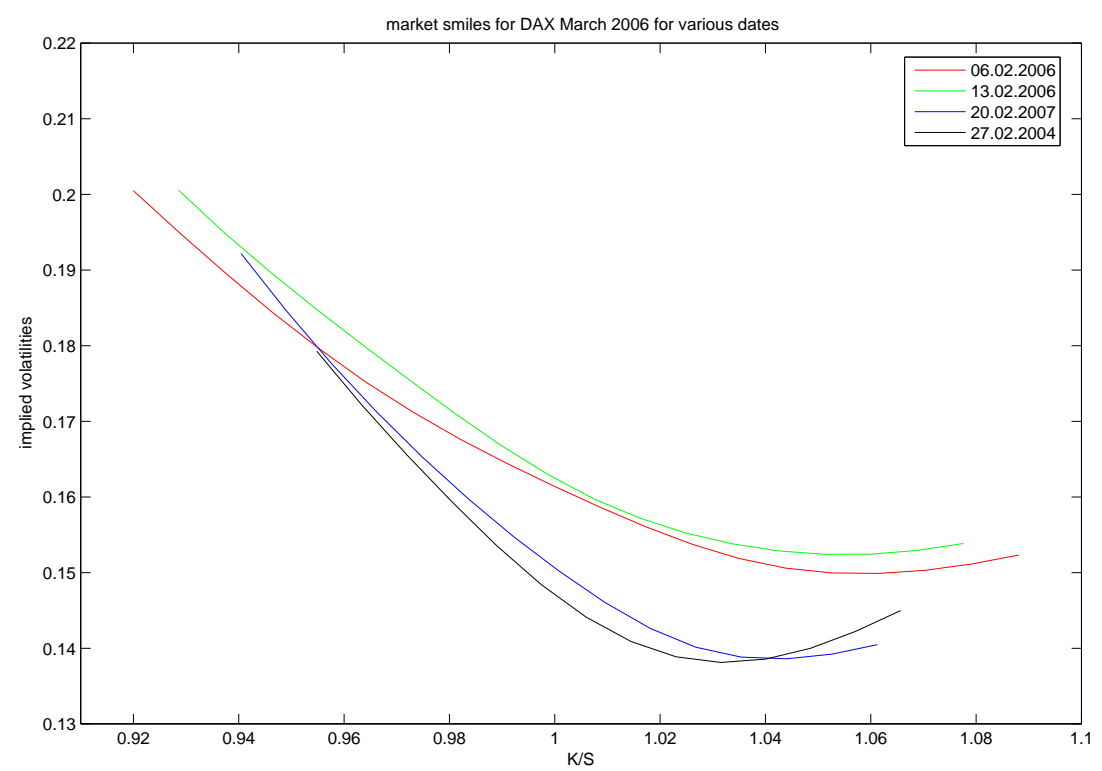

Figure 6.9: Market Volatility Smiles for for DAX March 2006 on various dates 


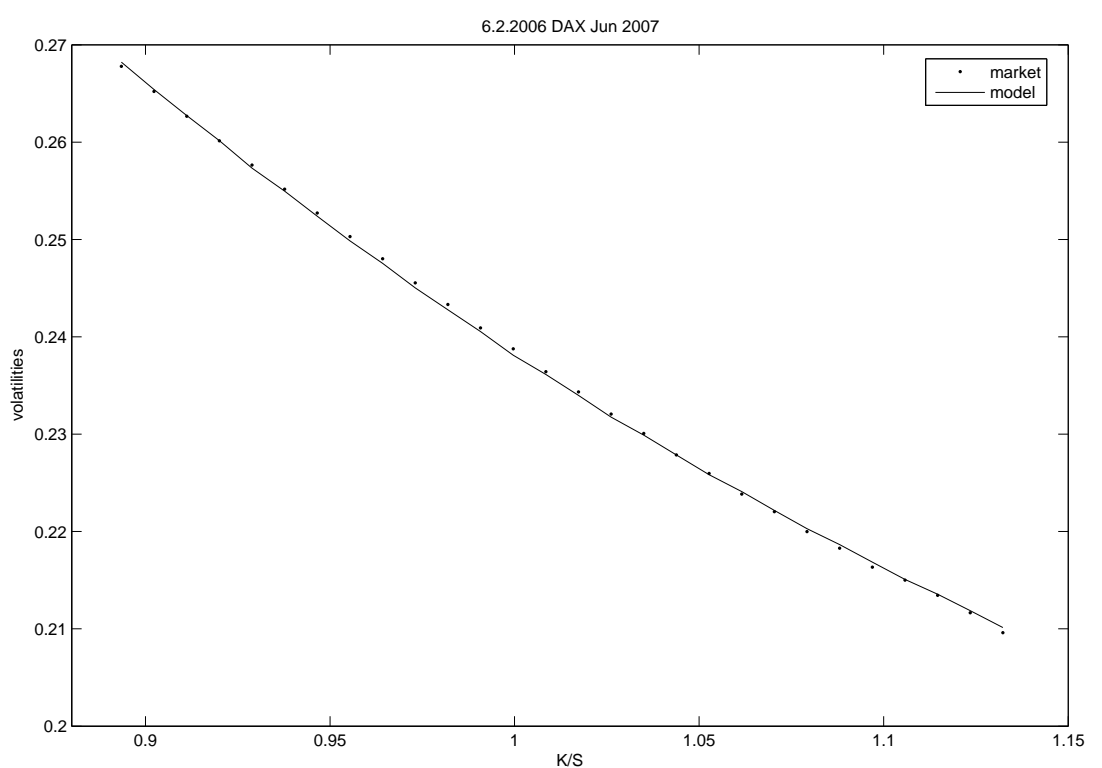

Figure 6.10: DAX June 2007, 6.2.2006

which means that Call options with those strikes are very strongly out of the money. Moreover the fitting errors for options prices are much smaller than those for implied volatilities. 


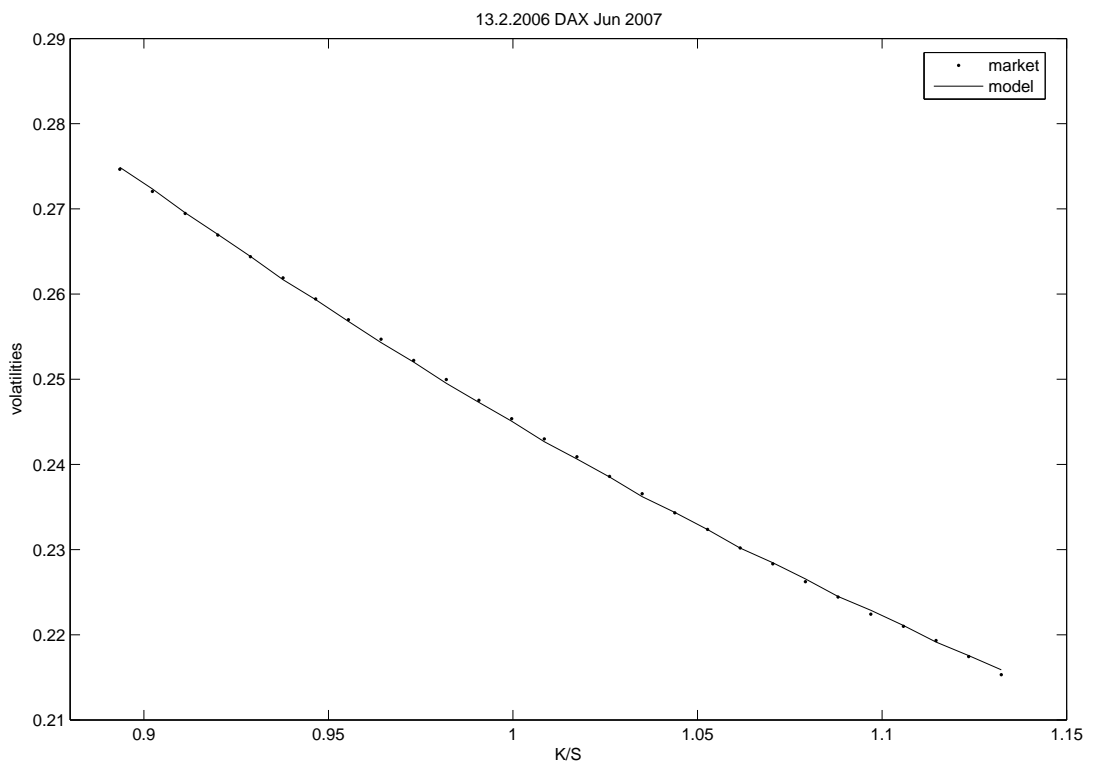

Figure 6.11: DAX June 2007, 13.2.2006

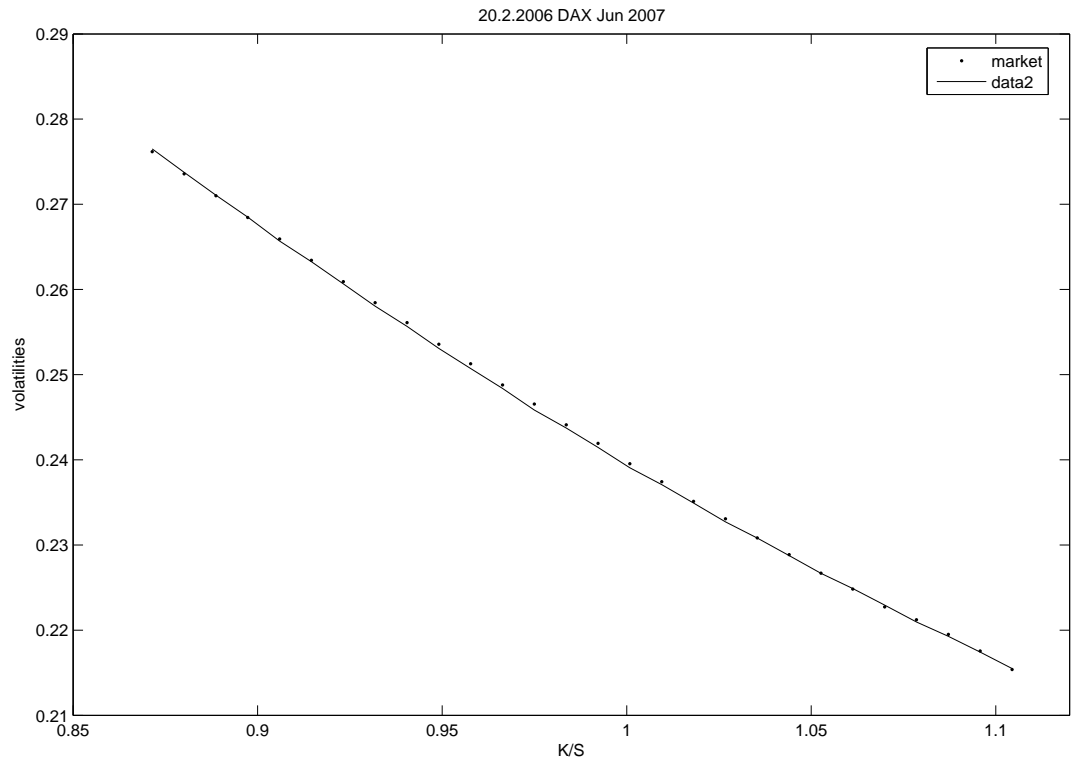

Figure 6.12: DAX June 2007, 20.2.2006 


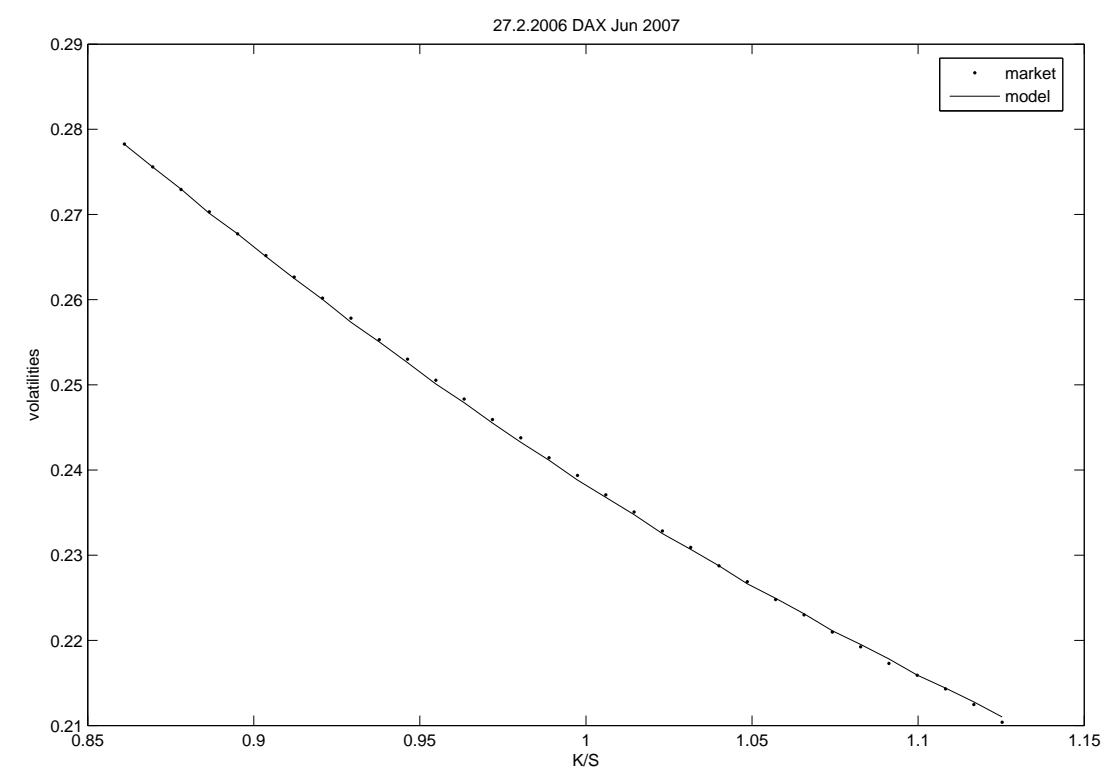

Figure 6.13: DAX June 2007, 27.2.2006

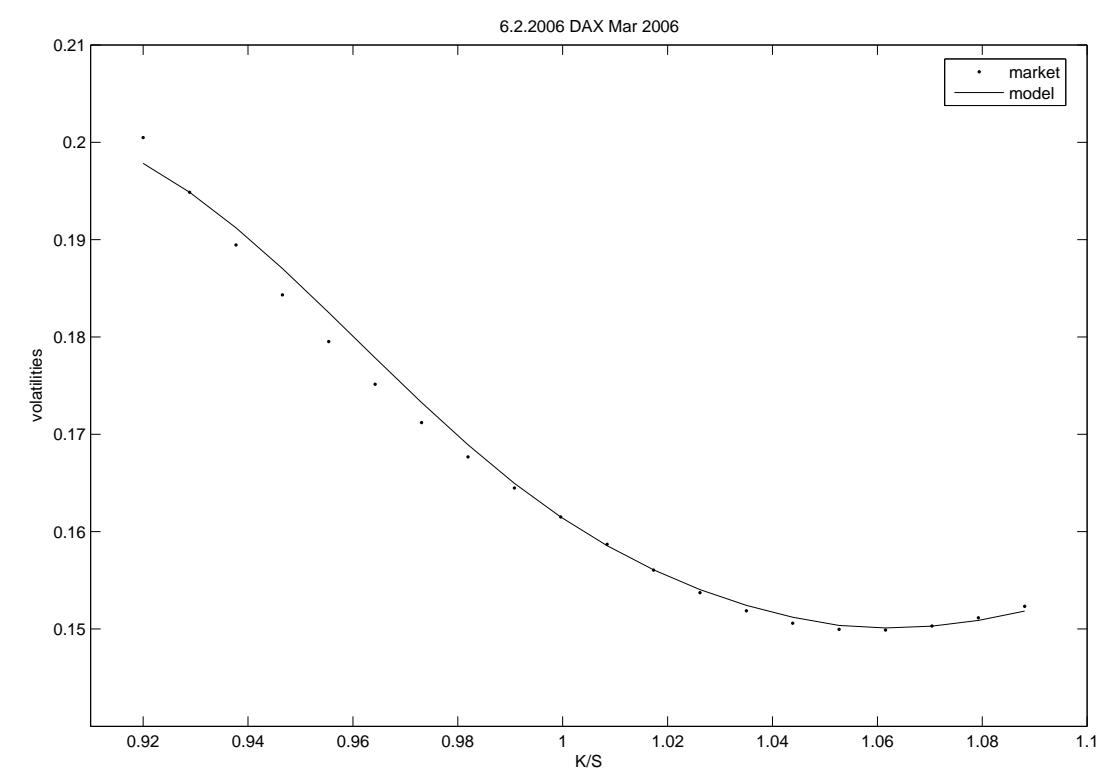

Figure 6.14: DAX March 2006, 6.2.2007 


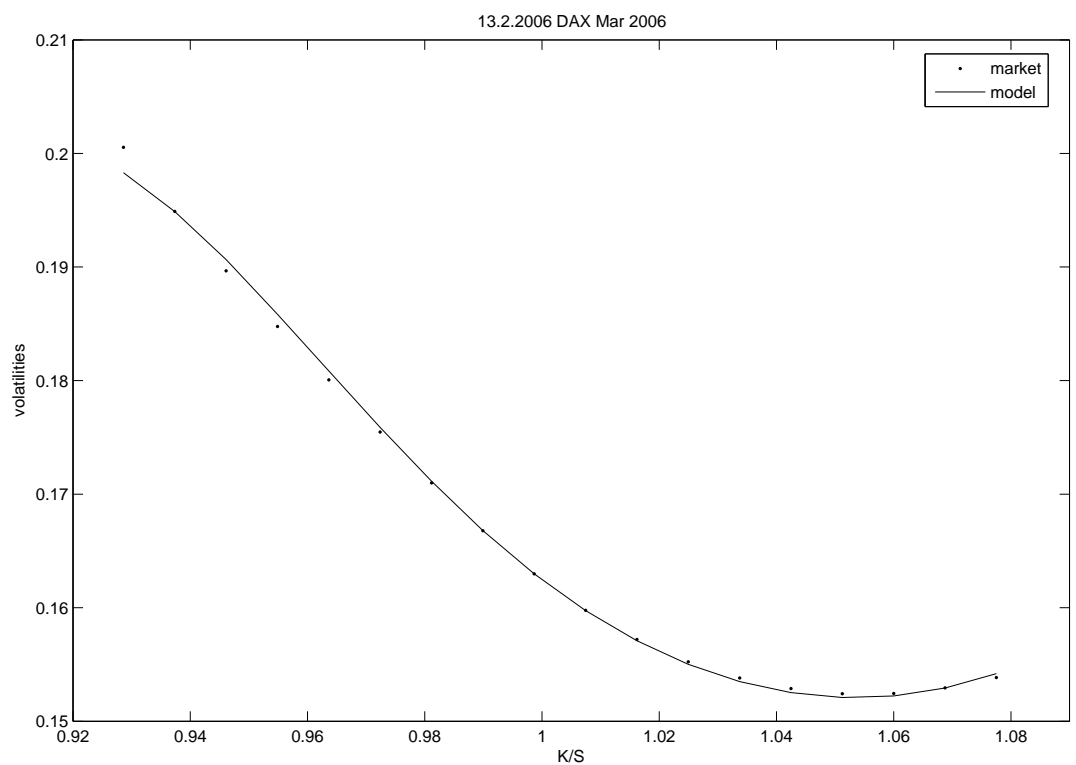

Figure 6.15: DAX March 2006, 13.2.2006

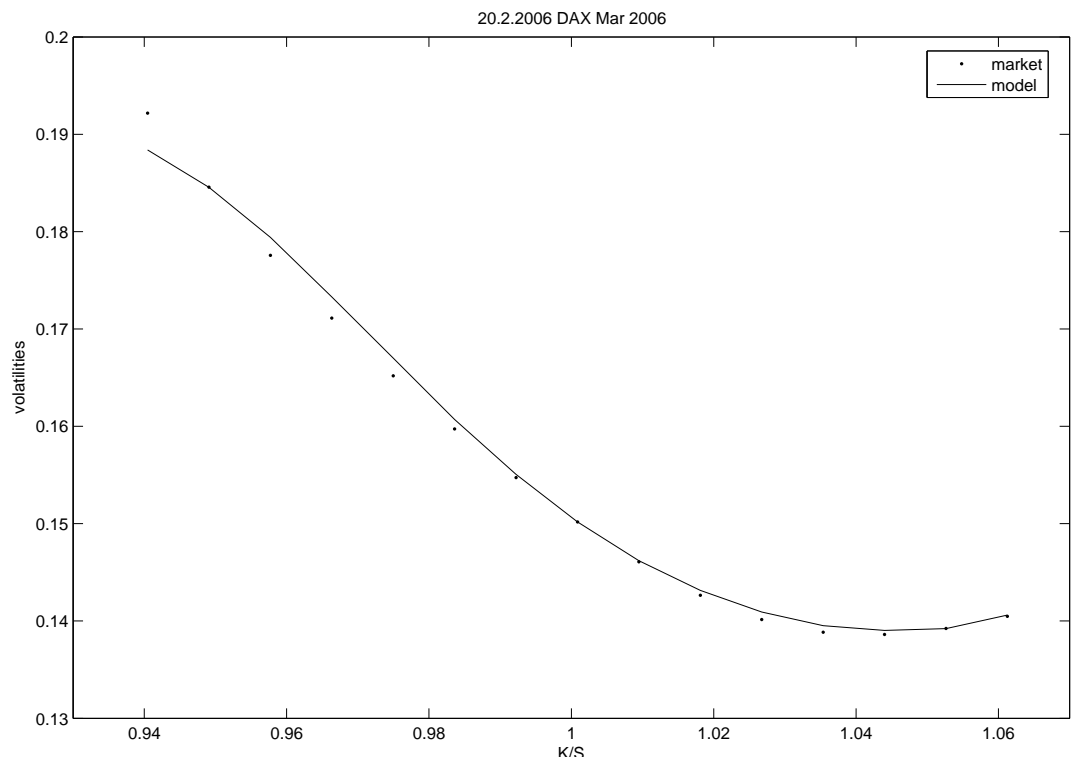

Figure 6.16: DAX March 2006, 20.2.2006 


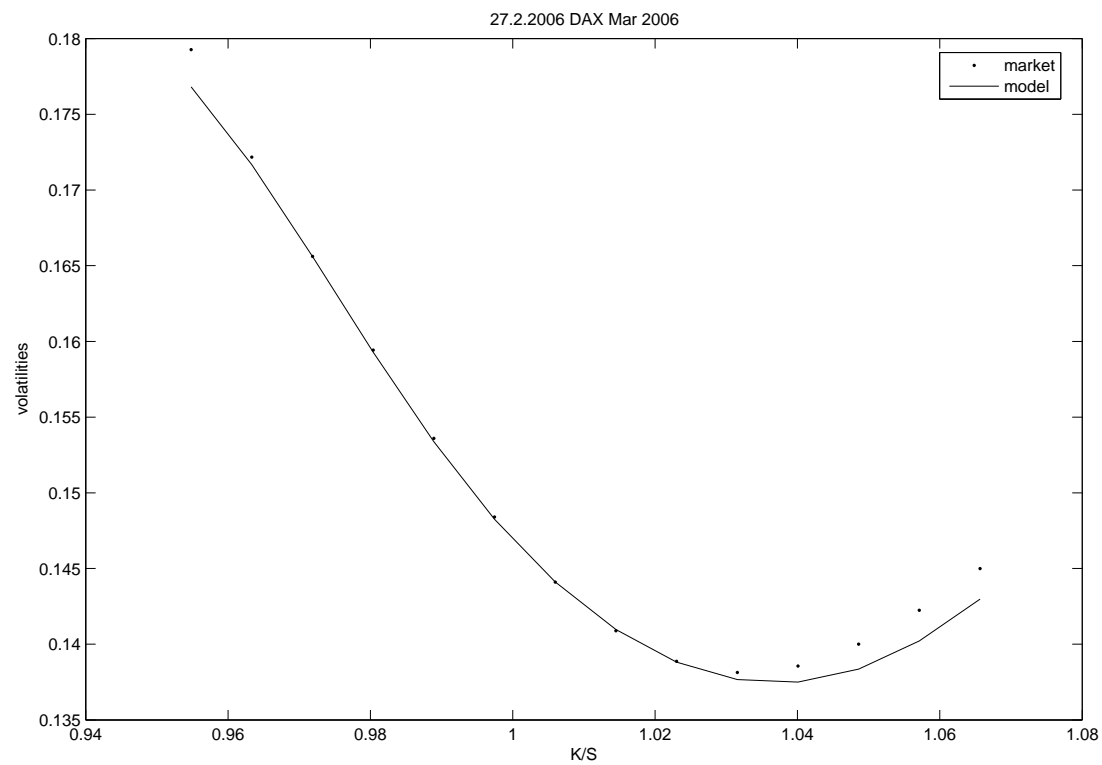

Figure 6.17: DAX March 2006, 27.2.2006

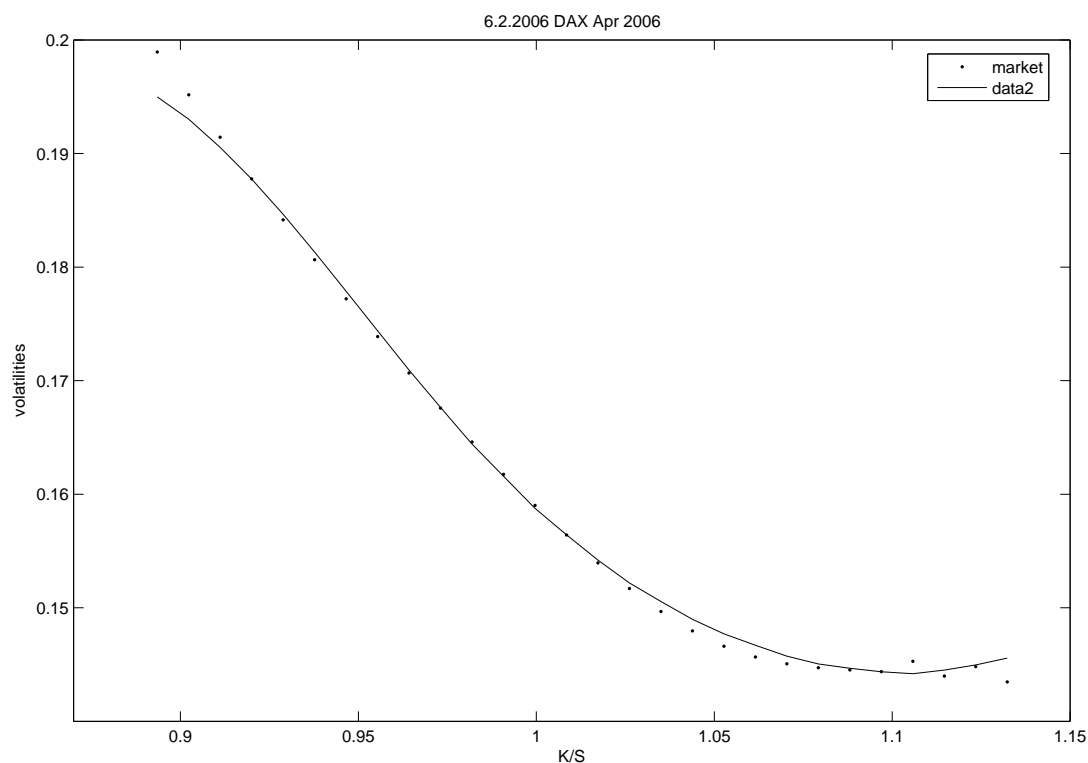

Figure 6.18: DAX April 2006, 6.2.2006 


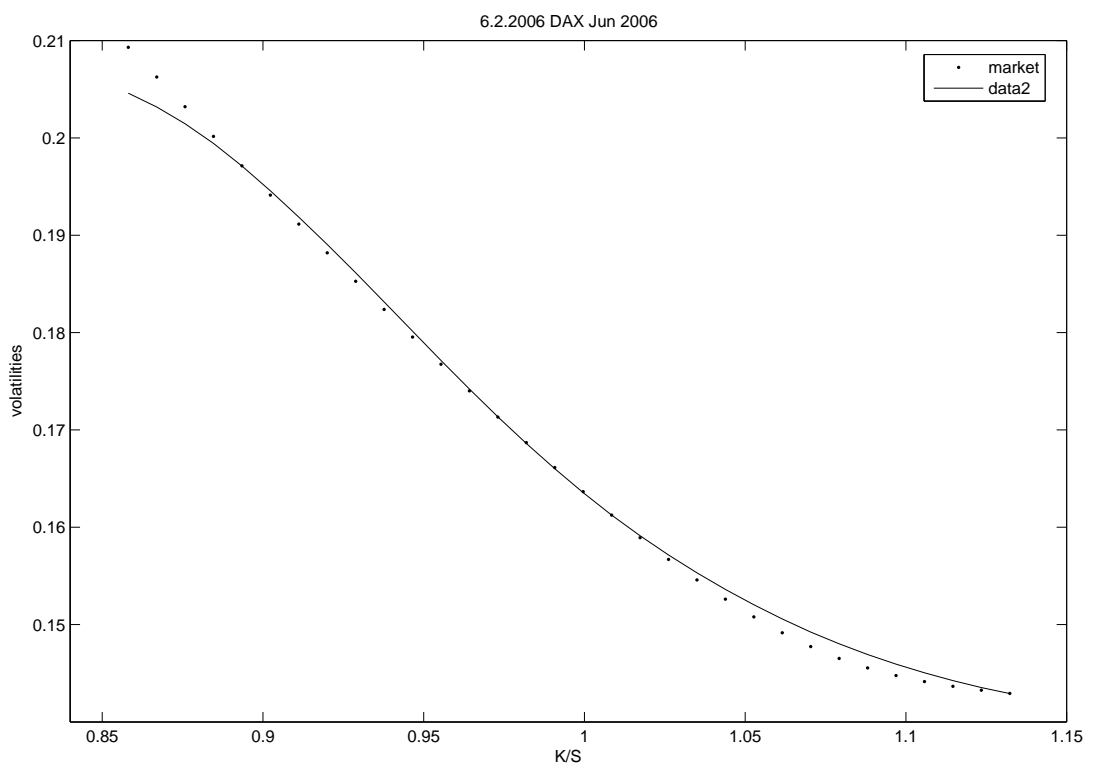

Figure 6.19: DAX June 2006, 6.2.2006

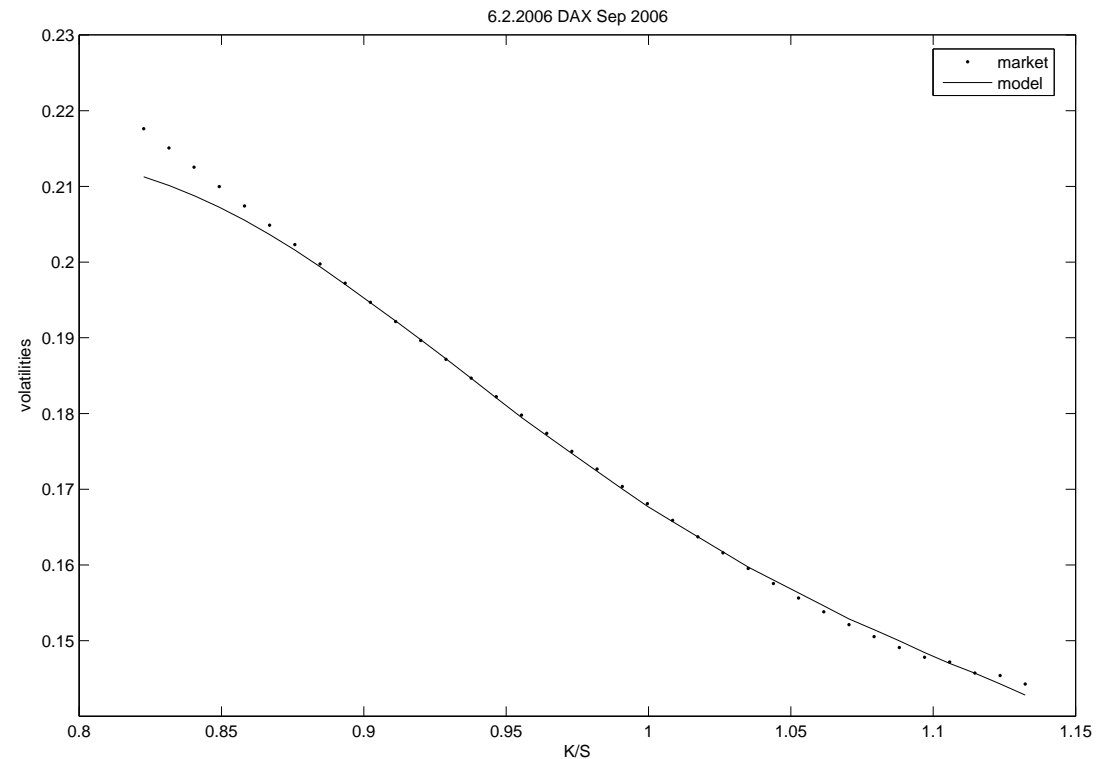

Figure 6.20: DAX September 2006, 6.2.2006 


\section{Chapter 7}

\section{Conclusions and Discussion}

In practice the hedging process does not satisfy the assumptions of the Black-Scholes model. Traders do not hedge continuously because of transaction costs and time constraints and often do not choose low risk over a high but risky profit. The goal of this thesis is to construct a model reflecting these aspects of option hedging in real life.

First we studied a model where a trader is allowed to rebalance his or her portfolio only at fixed dates. The trader's goal was to minimize the expectation of the squared difference between the value of the hedging portfolio and the option's payoff. We followed the theory of the mean-variance hedging of Schweizer [45]. For a special case of an underlying process with independent returns we have found an explicit solution for the minimization problem. For this special case we derived an analytical formula for the variance-optimal measure, a martingale measure with the smallest $L_{2}$ norm of its RadonNikodym derivative with respect to the real-world measure. As a corollary we were able to prove explicitly in a simpler way the result of Schweizer that the optimal initial capital is the expectation of the option payoff under the variance-optimal measure. We also proved that under the assumption of independent returns the variance optimal measure converges in the sense of $L_{2}$ convergence of Radon-Nikodym derivatives to the risk-neutral measure if the distances between hedging dates go to zero. This implies that the optimal initial capital converges to the Black-Scholes price.

Next we constructed a new optimization model, the model with cost of risk. In its setting the hedging dates are also fixed but instead of minimizing the distance between the portfolio value and the options' payoffs the trader's goal is to maximize the expected total profit from hedging a portfolio of options. The risk of the trader is controlled not by a utility function, which is very difficult to determine in practice, but by an extra bank account with an interest smaller than the interest rate on the regular bank account. This 
additional bank account is thought of as a capital reserve. According to the risk of his or her portfolio, the trader has to keep a certain amount of money in the reserve. Borrowing this amount under the risk-free rate and investing it under a lower rate in the reserve account makes the trader lose money.

We introduced the notion of indifference selling and buying prices of a portfolio of options in the presence of another portfolio of options. These prices depend on the trader's book and the buying and the selling prices are different, so the model is able to capture bid-ask spreads. For a special form of the risk function depending on the portfolio Greeks we found explicit solutions for the maximization problem and analytic formulas for the indifference prices. We proved that the indifference prices were adjusted binomial prices, with the adjustment term dependent on the expected differences between the risk functions of two portfolios: the trader's book and the portfolio to be priced. The indifference mid price was defined as the average of indifference buying and selling prices. The indifference mid prices were calibrated to the market. We were able to obtain a very good fit and the fitted parameters demonstrated a stable behavior.

The model with cost of risk is relatively simple and instead of using utility functions defines the trader's risk explicitly in terms of Greeks, observable market parameters. The main limitation of this model is that it cannot be fitted to the whole implied volatility surface at once. The model parameters are fitted per maturity. Thus it is impossible to use one set of parameters to calculate the risk of a whole trader's book, which usually contains options of more than one maturity.

Some further research may be done in the direction of a better fitting algorithm and a faster procedure for the pricing. The study of the properties of the fitted parameters such as their dependence on time might also be interesting. We suggest a few extensions of the model for consideration. We have already mentioned a possible introduction of the term structure on the model parameters.

Another one is incorporating dividends in the dynamics of the underlying price process. Including continuous dividends is pretty much straightforward. This will only affect the parameters of the binomial model and the formulae for the Greeks. Including continuous dividends allows to fit the model to the foreign exchange option prices. Discrete dividends are a bit more subtle, but it seems that adjusting the binomial tree and the Greeks in a proper way will solve the problem.

An important step is extending the model with cost of risk to pricing American and other path dependent options. Here some changes to the dynamics of the portfolio value might be necessary.

Some research may be done to find the continuous limit of the model. 
A first step is to keep the hedging dates fixed but to consider a continuous dynamics for the underlying process and a continuous adjustment to the amount on the reserve bank account. Letting the distance between the hedging dates go to zero will lead to continuous trading strategies and an Hamilton-Jacobi-Bellman partial differential equation for the solution of the optimization problem. 


\section{Bibliography}

[1] A. Bagchi. Optimal Control of Stochastic Systems. Prentice-Hall, 1993.

[2] S. Basak and A. Shapiro. Value-at-risk-based risk management: Optimal policies and asset prices. The Review of Financial Studies, 14 (2):371405, Summer 2001.

[3] Basel Committee on Banking Supervision. International Convergence of Capital Measurement and Capital Standards (updated April 1998), 1988. http://www.bis.org/publ/bcbsc111.pdf.

[4] Basel Committee on Banking Supervision. Basel II: International Convergence of Capital Measurement and Capital Standards: a Revised Framework, 2004.

[5] D. Bernoulli. Exposition of a new theory on the measurement of risk. Econometrica, 22 (1):23-36, January 1954. Translated from Latin into English by Dr. Louise Sommer, The American University, Washington, D. C., from 'Specimen Theoriae Novae de Mensura Sortis', Commentarii Academiae Scientiarum Imperialis Petropolitanae, Tomus V [Papers of the Imperial Academy of Sciences in Petersburg, Vol. V], 1738, pp. 175192.

[6] D.P. Bertsekas. Dynamic Programming and Stochastic Control. Academic Press Inc., New York, 1976.

[7] D.P. Bertsekas and S.E. Shreve. Stochastic Optimal Control: The Discrete Time Case. Athena Scientific, 1996.

[8] T. Björk. Arbitrage Theory in Continuous Time. New York, second edition, 2004.

[9] F. Black and M. Scholes. The pricing of options and corporate liabilities. Journal of Political Economy, 81 (3):637-654, 1973. 
[10] N. Bouleau and D. Lamberton. Residual risks and hedging strategies in Markovian markets. Stochastic Processes and their Applications, 33:131150, 1989.

[11] P. Carr, Geman H., and D.B. Madan. Pricing and hedging in incomplete markets. Journal of Financial Economics, 62(1):131-167, October 2001.

[12] M.H.A. Davis and A.R. Norman. Portfolio selection with transaction costs. Mathematics of Operations Research, 15(4):676-713, 1990.

[13] M.H.A. Davis, V.G. Panas, and T. Zariphopoulou. European option pricing with transaction costs. SIAM Journal of Control and Optimization, 31(2):470-493, 1993.

[14] F. Delbaen and W. Schachermayer. A general version of the fundamental theorem of asset pricing. Mathematische Annalen, 300:463-520, 1994.

[15] E. Derman and I. Kani. Riding on a smile. Risk, 7(2):32-39, 1994.

[16] D. Duffie and H.R. Richardson. Mean-variance hedging in continuous time. Annals of Applied Probability, 1:1-15, 1991.

[17] B. Dupire. Pricing with a smile. Risk, 7(1):18-20, 1994.

[18] H. Föllmer and D. Sonderman. Hedging of non-redundant contingent claims. In W. Hildenbrand and A. Mas-Colell, editors, Contributions to Mathematical Economics, pages 205-223. 1986.

[19] G.M. Fichtengol'c. The Fundamentals of Mathematical Analysis, volume 72-73 of International series of monographs on pure and applied mathematics. Oxford University Press: Pergamon Press, 1965. transl. (from Russ.) Osnovy matematicheskogo analiza. Moskva, 1960 by Sneddon, I.N. and Swinfen, A.

[20] A. Friedman. Stochastic Differential Equations and Applications. Academic Press, New York, San Francisco, London, 1975.

[21] C. Gourieroux, J.P. Laurent, and H. Pham. Mean-variance hedging and numeraire. Mathematical Finance, 8(3):179-200, 1998.

[22] S. Gugushvili. Dynamic programming and mean-variance hedging in discrete time. Georgian Mathematical Journal, 10(2):237-246, 2003.

[23] J.M. Harrison and S.R. Pliska. Martingales and stochastic integrals in the theory of continuous trading. Stochastic Processes and their Applications, 11:215-260, 1981. 
[24] S.L. Heston. A closed-form solution for options with stochastic volatility with applications to bond and currency options. The Review of Financial Studies, 6(2):327-343, 1993.

[25] S.D. Hodges and A. Neuberger. Optimal replication of contingent claims under transaction costs. Review of Futures Markets, 8:222-239, 1989.

[26] P. Hua and P. Wilmott. Value at risk and market crashes. http://www.finance.ox.ac.uk/file_links/finecon_papers/1999mf23.pdf.

[27] P. Jorion. Value at Risk: The Benchmark for Controlling Market Risk. McGraw-Hill Publishing Co., third edition, 2007.

[28] I. Karatzas and S. Shreve. Brownian Motion and Stochastic Calculus. Springer-Verlag, New York, 1988.

[29] J.P. Laurent and H. Pham. Dynamic programming and mean-variance hedging. Finance and Stochastics, 3:83-110, 1999.

[30] H. Markowitz. Portfolio Selection: Efficient Diversification of Investment. John Wiley and Sons, New York, 1959.

[31] F. Mercurio and T. Vorst. Option pricing with hedging at fixed trading dates. Applied Mathematical Finance, 3:135-158, 1996.

[32] R.C. Merton. Optimum consumption and portfolio rules in a continuoustime model. Journal of Economic Theory, 3:373-413, 1971.

[33] R.C. Merton. Theory of rational option pricing. Bell Journal of Economics and Management Science, 4(1):141-183, 1973.

[34] R.C. Merton. Option pricing when underlying stock returns are discontinuous. Journal of Financial Economics, 3:125-144, 1976.

[35] P. Monat and C. Stricker. Föllmer-Schweizer decomposition and meanvariance hedging for general claims. The Annals of Probability, 23:605628, 1995.

[36] M. Musiela and T. Zariphopoulou. An example of indifference prices under exponential preferences. Finance and Stochastics, 8:229-239, 2004.

[37] A. Pelsser and T. Vorst. The binomial model and the greeks. The Journal of Derivatives, Spring 1994.

[38] A. Pilpel and N.N. Taleb. Epistemology and risk management. http://www.fooledbyrandomness.com/LSE-Taleb-Pilpel.pdf. 
[39] P.A. Samuelson. Lifetime portfolio selection by dynamic stochastic programming. The Review of Economics and Statistics, 51 (3):239-246, August 1969.

[40] M. Schäl. On quadratic cost criteria for option hedging. Math. Oper. Research, 19:121-131, 1994.

[41] M. Schweizer. Mean-variance hedging for general claims. Annals of Applied Probability, 2:171-179, 1992.

[42] M. Schweizer. Option hedging for semimartingales. Stochastic Processes and their Applications, 37:339-363, 1993.

[43] M. Schweizer. Approximating random variables by stochastic integrals. The Annals of Probability, 22:1536-1575, 1994.

[44] M. Schweizer. Variance-optimal hedging in discrete time. Mathematics of Operations Research, 20:1-32, 1995.

[45] M. Schweizer. Approximation pricing and the variance-optimal martingale measure. The Annals of Probability, 24(1):206-236, March 1996.

[46] S.F. Stoikov. Pricing options from the point of view of a trader. To appear in International Journal of Theoretical and Applied Finance, 2007.

[47] A. Černý. Dynamic programming and mean-variance hedging in discrete time. Applied Mathematical Finance, 11(1):1-25, March 2003. 